\title{
A one-dimensional model for elastic ribbons: a little stretching makes a big difference
}

\author{
Basile Audoly \\ Laboratoire de Mécanique des Solides \\ CNRS, Institut Polytechnique de Paris \\ 91120 Palaiseau, France
}

\author{
SÉbastien Neukirch
}

Institut Jean le Rond d'Alembert

CNRS, Sorbonne Université, UMR 7190 75005 Paris, France

February 5, 2021

Starting from the theory of elastic plates, we derive a non-linear one-dimensional model for elastic ribbons with thickness $t$, width $a$ and length $\ell$, assuming $t \ll a \ll \ell$. It takes the form of a rod model with a special non-linear constitutive law accounting for both the stretching and the bending of the ribbon mid-surface. The model is asymptotically correct and can handle finite rotations. Two popular theories can be recovered as limiting cases, namely Kirchhoff's rod model for small bending and twisting strains, $\left|\kappa_{i}\right| \ll t / a^{2}$, and Sadowsky's inextensible ribbon model for $\left|\kappa_{i}\right| \gg t / a^{2}$; we point out that Sadowsky's inextensible model may be a poor approximation even for ribbons having a very thin cross-section, $a / t \sim 50 \gg 1$. By way of illustration, the one-dimensional model is applied ( $i$ ) to the lateral-torsional instability of a ribbon, showing good agreement with both experiments and finite-element shell simulations, and $(i i)$ to the stability of a twisted ribbon subjected to a tensile force. The non-convexity of the one-dimensional model is discussed; it is addressed by a convexification argument.

\section{INTRODUCTION}

Ribbons are thin elastic objects whose thickness $t$, width $a$ and length $\ell$ are all very different, $t \ll a \ll \ell$. Ribbons made up of materials that react to external stimuli such as illumination (YNI03, GMV +17 ) or temperature change (BNSH14), have been used to design lightweight structures that can respond to actuation: their flexibility and their thin geometry help turn the relatively small strain produced by actuation into a large-amplitude motion (RST +13 , WRW16). In addition, they are fabricated easily by cutting out a thin sheet of material.

Despite its relevance for applications, the elastic theory of ribbons is much less advanced than the theory of rods (which covers the case $a \sim t$ ). Elastic ribbons can be modeled as thin elastic shells but shell models are difficult to solve, both numerically and analytically. One-dimensional models for elastic ribbons are therefore desirable. There are two main models of this type available to date:

- the Kirchhoff rod model (Dil92), which is based on the classical theory of rods and can be justified from three-dimensional elasticity assuming $a / t=\mathcal{O}(1)$ : it is valid for moderate values of $a / t$, in a sense to be made precise later on. This model features linearly elastic constitutive laws, and makes use of the classical formula for the bending and twisting moduli $E I$ and $\mu J$ that are available from engineering textbooks.

- the Sadowsky ribbon model (Sad29), which assumes that the mid-surface of the ribbon is inextensible: it is valid for ribbons that have a large enough width-to-thickness ratio, $a / t \rightarrow \infty$, in a sense which will be made more precise later on.

The ribbon model of Wun62 extends that of Sadowsky to include the effect of the longitudinal gradient of twisting strain, remaining under the assumption that the mid-surface is inextensible: in the present work, we will view it as an extension of the Sadowsky model.

There are two important drawbacks to these existing one-dimensional models:

- their predictions do not agree. They predict both significantly different buckling loads (AS15), and significantly different non-linear solutions (MGN18). The bending law in Kirchhoff's model is linear, while it is essentially non-linear in Sadowsky's model (meaning that it is not possible to extract any linearized constitutive law from it). As a result, they have no domain of overlap, i.e., it is impossible to identify a range of parameters where these models agree.

- their respective domains of validity are not clearly characterized. There is currently no way to decide whether the Kirchhoff or Sadowsky models are applicable in a given situation. Even though Sadowsky's model is known to be valid in the limit $a / t \rightarrow \infty$, it has been reported to be inaccurate even in situations where $a / t$ is as large as $\sim 30$ (MGN18).

Our main contribution is to derive a new one-dimensional model for ribbons that solves these difficulties:

- it bridges the gap between the Kirchhoff and Sadowsky models, covering a range of parameter where neither one is applicable;

- it unifies them into a single framework, allowing them to be recovered as limiting cases;

- it provides a way of rationalizing their domains of validity in terms of a load-dependent dimensionless parameter $\bar{\kappa} \sim \kappa a^{2} / t$ where $\kappa$ is the typical value of the bending and twisting strain: the Kirchhoff and Sadowsky models are recovered when $|\bar{\kappa}| \ll 1$ and $|\bar{\kappa}| \gg 1$, respectively. The relevant parameter is $\bar{\kappa}$ and not $a / t$, and this explains why the Sadowsky model can perform poorly even for a thin ribbon, when $a / t$ is a large number. 
The present work builds up on the work of DA15 who have shown that Sadowsky's inextensible ribbon model can be formulated in a way that fits the non-linear theory of elastic rods: an inextensible ribbon is effectively a rod endowed with a non-linear constitutive law (that captures the deformations of the underlying plate model) and whose stiff bending mode is inhibited. In the present work, we extend this result by deriving an equivalent rod model for extensible ribbons. The main task is to derive its non-linear constitutive law from an extensible plate model. We observe that this derivation has to be done in the framework of non-linear elasticity, for the matching with the (nonlinear) Sadowsky model to be possible in the limit $\bar{\kappa} \rightarrow \infty$.

The derivation of a one-dimensional ribbon model is an asymptotic problem featuring several small parameters: two geometrical ones (the transverse and longitudinal aspect-ratios, $t / a \ll 1$ and $a / \ell \ll 1$ respectively), and one capturing the load intensity, say $\kappa \ell$, where $\kappa$ is the typical curvature of the solution produced by the load. Our results show that a key dimensionless parameter for ribbons is the combination

$$
\bar{\kappa} \sim \frac{\kappa a^{2}}{t}=\frac{(\kappa \ell)(a / \ell)}{(t / a)} .
$$

Depending on the value of $\bar{\kappa}$ (i.e., on the relative magnitude of $\kappa \ell, a / \ell$ and $t / a$ ), the limit models are obtained as follows:

- Sadowsky's inextensible ribbon model corresponds to $\bar{\kappa} \gg 1$ which can be rewritten as $(t / a) \ll(\kappa \ell)(a / \ell)$. This includes the typical case where $\kappa \ell=\mathcal{O}(1)$ (corresponding to solutions featuring finite rotations) and $t / a$ goes faster to zero than $a / \ell$, as is commonly achieved by letting $t / a$ go zero first and then letting $a / \ell$ go to zero.

- the Kirchhoff model corresponds to $\bar{\kappa} \ll 1$ which can be rewritten as $(\kappa \ell) \ll \frac{(t / a)}{(a / \ell)}$ : this sets a maximal load intensity, depending on the rod geometry, such that the linear constitutive laws from classical rod theory are applicable.

- our model also applies to intermediate values of $\bar{\kappa}$ and connects smoothly with the two limit models above.

Our ribbon model takes into account the extensibility of the ribbon mid-surface. It is derived by dimension reduction from a plate model, assuming $\bar{\kappa}=\mathcal{O}(1)$. We observe that $\bar{\kappa}=\mathcal{O}(1)$ implies $|\kappa a| \sim(t / a) \bar{\kappa} \ll 1$, i.e., the plate bends and twists by a small amount at the scale $a$ of the cross-section. In view of this, we use a simplified plate model, derived in section 6.3, as the starting point of the dimension reduction procedure. It is based on Föpplvon Kàrmàn-like geometric approximations for the strain in the local basis but it is still able to account for finite rotations with respect to a global frame. The simplified plate model is solvable analytically, which allows the onedimensional model to be found in closed form; this would not be the case if we had started from a geometrically exact-hence more complex - plate model.

The solution to the plate model is given, in terms of $\bar{\kappa}$, in the limit $a / \ell \ll 1$ and $t / a \ll 1$. It is asymptotically valid and does not make use of any ad hoc kinematic assumption. We proceed as follows:

- we first analyze homogeneous solutions of the plate model, which are such that the strain is invariant in the longitudinal direction. The strain energy density $W\left(\kappa_{2}, \kappa_{3}\right)$ per unit length of these homogeneous solutions are then obtained as a function of the macroscopic bending and twisting strains $\kappa_{2}$ and $\kappa_{3}$. The key role played by homogeneous solutions has been recognized in related dimension reduction problems, for example for ribbons subjected to pure bending (Shi92), tape springs (Wue54, Man73), linear beams (YH04) and nonlinear elastic rods (AL21).

- next, we use this energy density $W$ to define the strain energy potential $\Phi=\int_{0}^{\ell} W\left(\kappa_{2}(S), \kappa_{3}(S)\right) \mathrm{d} S$ of an equivalent rod model. Its equations of equilibrium are then obtained variationally. We observe that the classical theory of rods proceeds similarly, by defining the strain energy of a Kirchhoff rod as $\Phi=$ $\int \frac{E I}{2} \kappa^{2}(S) \mathrm{d} S$, where the integrand $\frac{E I}{2} \kappa^{2}$ captures the strain energy density of circular solutions, having uniform curvature $\kappa$.

- we illustrate how this equivalent rod model can be applied, by solving two typical 'structure' problems, in which the strain measures $\kappa_{2}(S)$ and $\kappa_{3}(S)$ are no longer invariant in the longitudinal direction-they are still slowly variable: they vary over a characteristic length that is much larger than the width $a$.

A similar approach has been used in earlier work on dimension reduction: it is called the zeroth-order model in the work of Hod06. We emphasize that the proposed approach is asymptotically exact. Indeed, LA20, AL21 have shown that the energy $\Phi$ is the dominant contribution in a systematic expansion of a one-dimensional strain energy in powers of the successive gradients of the strain measures $\kappa_{2}(S)$ and $\kappa_{3}(S)$. Here, we limit our attention to the dominant contribution $\Phi=\int_{0}^{\ell} W\left(\kappa_{2}(S), \kappa_{3}(S)\right) \mathrm{d} S$. Pushing the expansion further so as to include the strain gradients $\kappa_{2}^{\prime}(S)$ and $\kappa_{3}^{\prime}(S)$ in the strain energy $\Phi$ is significantly more difficult.

It is worth emphasizing that the present work explores a different direction than thin-walled beam models, such as the popular one due to Vlasov (Vla61). Our model features non-linear constitutive relations and ignores the gradient effect. Conversely, thin-walled beams models capture the gradient effect but typically make use of linear constitutive relations.

The existing justifications of one-dimensional models for ribbons fall in two classes:

- in the inextensible case, the inextensibility is used as the first step in the reduction procedure; this typically allows the developable, hence ruled, mid-surface to be parameterized based on the center-line (Wun62). There is no obvious way to extend this approach beyond the inextensible case. In the work of ADK17, the midsurface developability is replaced by a more general condition of isometry with respect to a prescribed metric, and the latter is strictly enforced in the limit model as well. 
a)

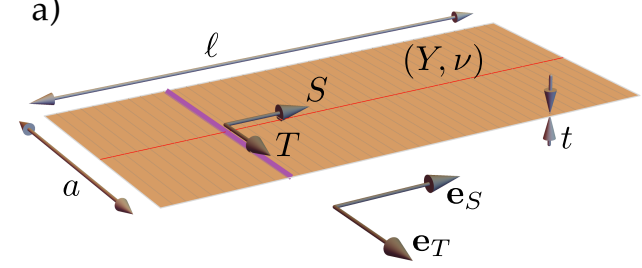

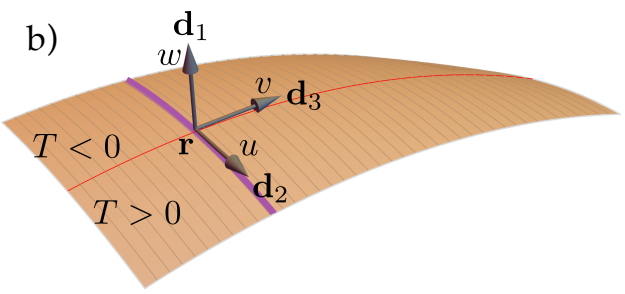

Figure 2.1. An elastic ribbon in (a) reference and (b) actual configurations. A particular cross-section with coordinate $S$ is highlighted in purple color.

- a few papers have carried out dimension reduction without assuming mid-surface inextensibility, by properly considering the two small parameters $t / a$ and $a / \ell$. To the best of our knowledge, their outcome has been limited to linear beam models so far, either because the starting point was linear elasticity (FMP04), or because they used von Kármán kinematic assumptions which are satisfied as long as $|\bar{\kappa}| \ll 1$ only (FHMP 18 ). In the work of FMP12, various scaling regimes have been studied but not the regime $|\bar{\kappa}|=\mathcal{O}(1)$ that is of interest here.

Besides, a number of authors have derived one-dimensional models starting from kinematic assumptions, both for ribbons (BTQF14, GSD16, TBNV19, GB05) and for the related problem of tape springs (PBCG16, BFV20); in the present work, our focus is instead on asymptotically exact models.

\section{MAIN RESULT: ONE-DIMENSIONAL MODEL FOR AN ELASTIC RIBBON}

We consider an elastic ribbon made up of an isotropic, linearly elastic material with Young's modulus $Y$ and Poisson's ratio $\nu$. The ribbon dimensions are $t$ (thickness), $a$ (width) and $\ell$ (length), see figure 2.1a. The longitudinal and transverse coordinates in the reference configuration are denoted as $S$ and $T$, respectively; they vary in the ranges $0 \leqslant S \leqslant \ell$ and $-a / 2 \leqslant T \leqslant a / 2$. We denote by $\boldsymbol{e}_{T}$ and $\boldsymbol{e}_{S}$ the unit vectors aligned with the coordinates $T$ and $S$ in reference configuration.

\subsection{Kinematic analysis}

The deformed configuration shown in figure $2.1 \mathrm{~b}$ is analyzed as follows. The centroid of the cross-section with coordinate $S$ is denoted as $\boldsymbol{r}(S)$. The curve $S \mapsto \boldsymbol{r}(S)$ is referred to as the center-line of the ribbon, and is shown in red in figure 2.1b. To capture the local orientation of the ribbon, we define a unit vector $\boldsymbol{d}_{1}(S),\left|\boldsymbol{d}_{1}(S)\right|=1$, which is roughly normal to the ribbon surface at $\boldsymbol{r}(S)$-an accurate definition of $\boldsymbol{d}_{1}(S)$ will be given later on in equation (6.3). For the moment it suffices to know that $\boldsymbol{d}_{1}(S)$ is perpendicular to the center-line, $\boldsymbol{d}_{1}(S) \cdot \boldsymbol{r}^{\prime}(S)=0$. The orientation of the unit normal $\boldsymbol{d}_{1}(S)$ is chosen so that $\boldsymbol{d}_{1}(S)=\boldsymbol{e}_{T} \times \boldsymbol{e}_{S}$ in the reference configuration. In the case of non-orientable ribbons such as a Möbius strip, an imaginary cut must be introduced in the reference configuration, where the normal $\boldsymbol{d}_{1}(S)$ is allowed to flip.

In terms of the material tangent $\boldsymbol{r}^{\prime}(S)$, we introduce the unit tangent $\boldsymbol{d}_{3}(S)=\frac{\boldsymbol{r}^{\prime}(S)}{\left|\boldsymbol{r}^{\prime}(S)\right|}$ and the axial strain $\varepsilon(S)=\left|\boldsymbol{r}^{\prime}(S)\right|-1$ :

$$
\boldsymbol{r}^{\prime}(S)=(1+\varepsilon(S)) \boldsymbol{d}_{3}(S) \text {. }
$$

As $\boldsymbol{d}_{1}(S)$ and $\boldsymbol{d}_{3}(S)$ are mutually perpendicular unit vectors for any value of $S$, we can form a direct orthonormal basis $\left(\boldsymbol{d}_{1}(S), \boldsymbol{d}_{2}(S), \boldsymbol{d}_{3}(S)\right)$ by introducing a third vector $\boldsymbol{d}_{2}(S)=\boldsymbol{d}_{3}(S) \times \boldsymbol{d}_{1}(S)$ :

$$
\forall S \quad \boldsymbol{d}_{i}(S) \cdot \boldsymbol{d}_{j}(S)=\delta_{i j}
$$

where $\delta_{i j}$ denotes the Kronecker symbol and latin indices, such as $i$ and $j$ run by default along all directions of space, $i, j \in\{1,2,3\}$. The vectors $\boldsymbol{d}_{i}(S)$ are known as the directors in the theory of rods. With our conventions, $\boldsymbol{d}_{2}(S)$ points to the $T>0$ side of the ribbon, see figure $2.1 \mathrm{~b}$.

The center-line $\boldsymbol{r}(S)$ and orthonormal frame of directors $\boldsymbol{d}_{i}(S)$ are the fundamental kinematic unknowns parameterizing the deformed ribbon. The same set of unknowns is used in the classical theory or rods, and we use in the following the standard kinematic analysis of framed curves $S \mapsto\left(\boldsymbol{r}(S), \boldsymbol{d}_{i}(S)\right)$. We observe that the orthonormal character of directors frame $\boldsymbol{d}_{i}(S)$ implies the existence of a vector $\boldsymbol{\kappa}(S)$, called the rotation gradient or the Darboux vector, such that $\boldsymbol{d}_{i}^{\prime}(S)=\boldsymbol{\kappa}(S) \times \boldsymbol{d}_{i}(S)$ for any $S$. Denoting as $\kappa_{i}(S)$ its components in the directors basis, we have

$$
\boldsymbol{d}_{i}^{\prime}(S)=\boldsymbol{\kappa}(S) \times \boldsymbol{d}_{i}(S) \text { where } \boldsymbol{\kappa}(S)=\kappa_{i}(S) \boldsymbol{d}_{i}(S) .
$$

The quantities $\kappa_{1}(S)$ and $\kappa_{2}(S)$ are the bending strains, while $\kappa_{3}(S)$ is the twisting strain. Overall, the macroscopic strain measures for the ribbon are the stretching strain $\varepsilon(S)$ from equation (2.1) and the bending and twisting strains $\kappa_{i}(S)$ from equation (2.3). They will enter into the one-dimensional model for the ribbon.

We close this kinematic analysis by two remarks. First, we emphasize that the directors serve a single purpose, which is to keep track of the average twist angle of the cross-sections about the tangent, where the average is taken over each cross-section. The cross-sections may well be curved, as depicted in figure $2.1 \mathrm{~b}$ : it is not assumed that the cross-section with coordinate $S$ is contained in a plane perpendicular to $\boldsymbol{d}_{1}(S)$.

Second, the alignment constraint between the center-line tangent $\boldsymbol{r}^{\prime}(S)$ and the director $\boldsymbol{d}_{3}$ in equation (2.1) defines the so-called Euler-Bernoulli family of rod models in the classical theory of rods. In the literature on the derivation of rod models from three-dimensional elasticity, this condition (2.1) is often introduced as an ad hoc approximation. By contrast, in the present work, the Euler-Bernoulli kinematic condition (2.1) is merely a definition of the director $\boldsymbol{d}_{3}$. It entails no a priori restriction on the microscopic displacement, which will be rigorously determined in section 6 by solving the equations of elastic equilibrium. 


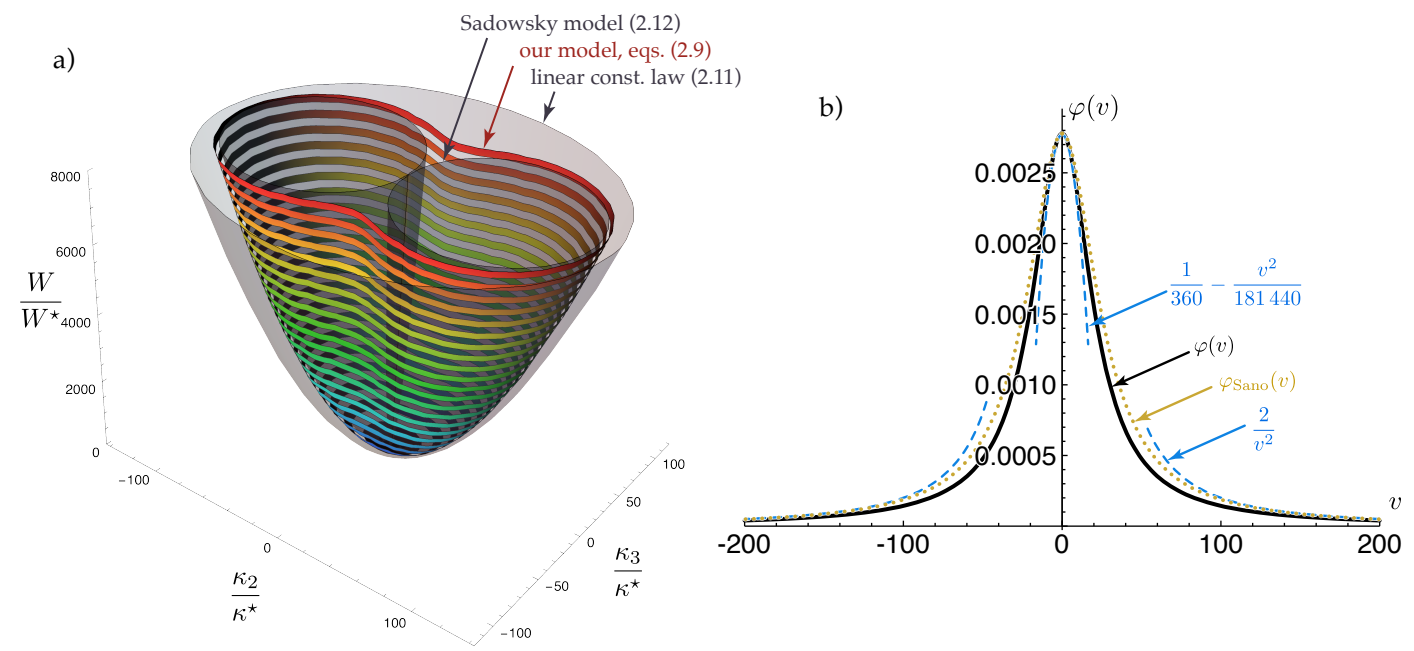

Figure 2.2. (a) Scaled strain energy $W / W^{\star}$, as predicted by equation (2.9) for $\nu=0.4$, as a function of the scaled bending strain $\bar{\kappa}_{2}=\kappa_{2} / \kappa^{\star}$ and of the scaled twisting strain $\bar{\kappa}_{3}=\kappa_{3} / \kappa^{\star}$. The strain energy of our equivalent rod model for the ribbon (colored surface) is well approximated by a quadratic strain energy $(2.11)$ when both $\kappa_{2} / \kappa^{\star}$ and $\kappa_{3} / \kappa^{\star}$ are small (outer transparent paraboloid) and by Sadowsky energy (2.12) when $\kappa_{2} / \kappa^{\star}$ is large (double inner tube). (b) Graph of the function $\varphi(v)$ defined in equation (2.8) (black solid curve) as well as its equivalents for small and large strain (blue dashed curves) and its approximation $\varphi$ sano from equation (3.6) (dotted orange curve).

\subsection{Energy formulation of the one-dimensional model}

In section 6 , the equivalent rod model for the ribbon is derived $(i)$ by identifying natural scaling laws for the strain $\varepsilon, \kappa_{i}$ in terms of the ribbon width $a$ and thickness $t$, based on the scaling arguments from the introduction, $(i i)$ by introducing a thin plate model based on kinematic approximations that are suited to this scaling regime, and (iii) by applying a dimension reduction procedure to derive an equivalent one-dimensional (rod) model from this two-dimensional (plate) model.

The result of this dimension reduction procedure is an equivalent rod model made up of the following components:

- the standard kinematic definitions listed in equations (2.1-2.3),

- two kinematic constraints enforcing both inextensibility and unbendability about $\boldsymbol{d}_{1}(S)$,

$$
\Phi=\int_{0}^{\ell} W\left(\kappa_{2}(S), \kappa_{3}(S)\right) \mathrm{d} S
$$

- a non-linear elastic strain energy

$$
\forall S\left\{\begin{aligned}
\varepsilon(S) & =0 \\
\kappa_{1}(S) & =0,
\end{aligned}\right.
$$

where the strain energy density $W\left(\kappa_{2}, \kappa_{3}\right)$ writes

$$
W\left(\kappa_{2}, \kappa_{3}\right)=\frac{1}{2} \frac{Y a t^{3}}{12} \kappa_{2}^{2}+\frac{1}{2} \frac{Y a t^{3}}{6(1+\nu)} \kappa_{3}^{2}+\frac{1}{2} \frac{Y a^{5} t}{2}\left(\nu \kappa_{2}^{2}+\kappa_{3}^{2}\right)^{2} \varphi\left(\frac{\kappa_{2}}{\kappa^{\star}}\right) .
$$

Here, $\kappa^{\star}$ is the characteristic curvature strain

and $\varphi(v)$ is the numerical function

$$
\kappa^{\star}=\frac{1}{\left[12\left(1-\nu^{2}\right)\right]^{1 / 2}} \frac{t}{a^{2}}
$$

$$
\varphi(v)=\frac{4}{v^{2}}\left(\frac{1}{2}-\frac{\cosh \sqrt{\frac{|v|}{2}}-\cos \sqrt{\frac{|v|}{2}}}{\sqrt{\frac{|v|}{2}}\left(\sinh \sqrt{\frac{|v|}{2}}+\sin \sqrt{\frac{|v|}{2}}\right)}\right) .
$$

These equations define the equivalent rod model completely.

The function $\varphi(v)$ is plotted in figure $2.2 \mathrm{~b}$. It is even and $\mathcal{C}^{\infty}$-smooth over the real interval $v \in \mathbb{R}$. The singularity at $v=0$ in the expression above is apparent and $\varphi$ can be extended by continuity near $v=0$ as

$$
\varphi(v)=\frac{1}{360}-\frac{v^{2}}{181440}+\mathcal{O}\left(v^{4}\right)
$$

Even though mid-surface of the underlying plate model is allowed to stretch, this stretchability is not exposed in the one-dimensional model, and the latter is effectively inextensible, see equation (2.4). This point is further discussed in section 6.4.

\subsection{Strain energy with dimensionless variables}

An equivalent expression of the strain energy density can be derived in terms of the dimensionless bending and twisting strain

$$
\bar{\kappa}_{2}(S)=\frac{\kappa_{2}(S)}{\kappa^{\star}}, \quad \bar{\kappa}_{3}(S)=\frac{\kappa_{3}(S)}{\kappa^{\star}}
$$


(a)

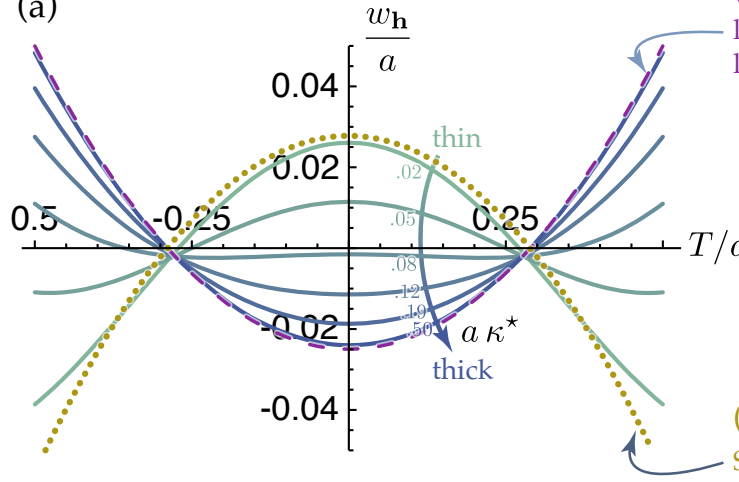

(b)

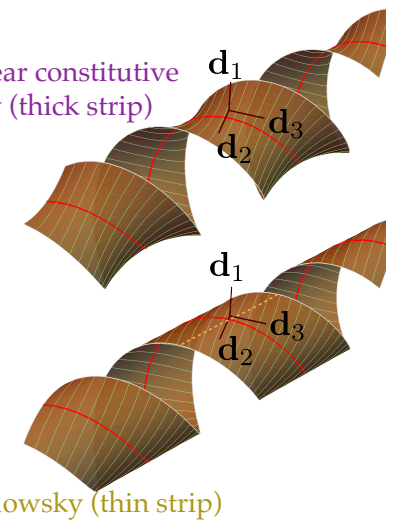

Figure 2.3. (a) Dependence of the deflection $w_{\boldsymbol{h}}(T)$ on the ribbon thickness $t$, for a helical solution. The scaled deflection $w_{h} / a$ is plotted as a function of the scaled transverse coordinate $T / a$ based on equation (2.13). The width $a$ and the macroscopic strain $\kappa_{2}$ and $\kappa_{3}$ are held constant, with $a \kappa_{2}=-1.5$ and $a \kappa_{3}=1$; Poisson's ratio is set to $\nu=0.4$. The various curves correspond to different values of the thickness $t$, with $a \kappa^{\star} \sim t / a$ ranging from 0.02 to 0.5. Darker curves correspond to thicker ribbons, and match the prediction of the linearly elastic model (3.3) (dashed curve) when $a \kappa^{\star}$ is large enough. Lighter curves correspond to thinner ribbons, and match the prediction of Sadowsky's theory (3.4) (dotted curve) when $a \kappa^{\star}$ is small enough. (b,c) Reconstruction of deformed ribbon in both limit cases: in the thick limit the ribbon displays anti-clastic curvature, as in the linearly elastic model; while in the thin limit the ribbon is developable, as in Sadowsky theory (see section 3 for details).

by inserting the expressions of $\bar{\kappa}_{2}$ and $\bar{\kappa}_{3}$ into equation (2.6). This yields

where

$$
W\left(\kappa_{2}, \kappa_{3}\right)=W^{\star} \times \frac{1}{2}\left[\bar{\kappa}_{2}^{2}\left(1-\nu^{2}\right)+2 \bar{\kappa}_{3}^{2}(1-\nu)+\left(\nu \bar{\kappa}_{2}^{2}+\bar{\kappa}_{3}^{2}\right)^{2} \frac{\varphi\left(\bar{\kappa}_{2}\right)}{2}\right]
$$

$$
W^{\star}=\frac{Y t^{5}}{a^{3}\left(12\left(1-\nu^{2}\right)\right)^{2}}
$$

is a natural scale for the strain energy. The alternative form (2.9) of the strain energy is slightly more convenient to discuss orders of magnitudes and limit cases.

From equation (2.9), two interesting limit cases can be obtained:

- when the bending and twisting strains are small, $\left|\kappa_{2}\right| \ll \kappa^{\star}$ and $\left|\kappa_{3}\right| \ll \kappa^{\star}$, i.e., $\left|\bar{\kappa}_{2}\right| \ll 1$ and $\left|\bar{\kappa}_{3}\right| \ll 1$, the last term in the square bracket in equation (2.9) is negligible: this yields a quadratic approximation to the strain energy

$$
\begin{aligned}
W & \approx W^{\star} \times \frac{1}{2}\left[\bar{\kappa}_{2}^{2}\left(1-\nu^{2}\right)+2 \bar{\kappa}_{3}^{2}(1-\nu)\right] \\
& \approx \frac{1}{2} \frac{Y a t^{3}}{12} \kappa_{2}^{2}+\frac{1}{2} \frac{Y a t^{3}}{6(1+\nu)} \kappa_{3}^{2},
\end{aligned}
$$

which coincides with the prediction of the Kirchhoff theory for a beam with rectangular cross-section dimensions $a \times t$, in the limit $t \ll a$, see the book of Lur05 for example. In what follows, we will refer to this Kirchhoff's model as the linearly elastic model;

- in the opposite limit of a large bending strain, $\left|\kappa_{2}\right| \gg \kappa^{\star}$, i.e., $\left|\bar{\kappa}_{2}\right| \gg 1$, the asymptotic behaviour $\varphi\left(\bar{\kappa}_{2}\right) \approx 2 / \bar{\kappa}_{2}^{2}$ can be inserted into equation (2.9) to yield

$$
\begin{aligned}
W & \approx W^{\star} \times \frac{1}{2}\left[\bar{\kappa}_{2}^{2}+2 \bar{\kappa}_{3}^{2}+\frac{\bar{\kappa}_{3}^{4}}{\bar{\kappa}_{2}^{2}}\right] \\
& \approx W^{\star} \times \frac{1}{2} \frac{\left(\bar{\kappa}_{2}^{2}+\bar{\kappa}_{3}^{2}\right)^{2}}{\bar{\kappa}_{2}^{2}} \quad \text { (Sadowsky, developable ribbon) } \\
& \approx \frac{1}{2} \frac{Y a t^{3}}{12\left(1-\nu^{2}\right)} \frac{\left(\kappa_{2}^{2}+\kappa_{3}^{2}\right)^{2}}{\kappa_{2}^{2}}
\end{aligned}
$$

which is the strain energy density defining Sadowsky's inextensible ribbon model.

These two limit cases are shown using the semi-transparent surfaces in the energy plot of figure 2.2. They are discussed further in section 3.

\subsection{Microscopic displacement}

The deflection $w$ is defined as the displacement of a point on the mid-surface of the plate, measured along the normal $\boldsymbol{d}_{1}(S)$. It is found in section 6 in terms of the local value $\boldsymbol{h}=\left(\kappa_{2}(S), \kappa_{3}(S)\right)$ of the macroscopic strain as $w(S, T)=w_{\boldsymbol{h}}(T)$, where

$$
w_{\boldsymbol{h}}(T)=a^{2} \kappa^{\star} \times\left[\cos \frac{q T}{a} \cosh \frac{q T}{a}\left(c_{1}+c_{2}\right) c_{3}+\sin \frac{q T}{a} \sinh \frac{q T}{a}\left(c_{1}-c_{2}\right) c_{3}-\frac{\kappa_{3}^{2}}{2 \kappa_{2} \kappa^{\star}}\left(\frac{1}{12}-\frac{T^{2}}{a^{2}}\right)\right] .
$$

Here, $c_{1}=-\sinh \frac{q}{2} \cos \frac{q}{2}, c_{2}=\cosh \frac{q}{2} \sin \frac{q}{2}, c_{3}=\frac{2\left(\nu \kappa_{2}^{2}+\kappa_{3}^{2}\right)}{\kappa_{2}^{2} \times \operatorname{sign}\left(\kappa_{2}\right)(\sin q+\sinh q)}$ and $q=\sqrt{\frac{\left|\kappa_{2}\right|}{2 \kappa^{\star}}}$. The deflection (2.13) is plotted in figure 2.3 for helical ribbons ( $\kappa_{2}$ and $\kappa_{3}$ are constant) having different thickness values $t$. 
For later reference, the curvature tensor of the mid-surface is found in section 6, equation (6.13), as

$$
\boldsymbol{B}(S, T)=\boldsymbol{B}_{\boldsymbol{h}}(T)=\kappa_{2} \boldsymbol{e}_{S} \otimes \boldsymbol{e}_{S}-\kappa_{3}\left(\boldsymbol{e}_{S} \otimes \boldsymbol{e}_{T}+\boldsymbol{e}_{T} \otimes \boldsymbol{e}_{S}\right)+w_{\boldsymbol{h}}^{\prime \prime}(T) \boldsymbol{e}_{T} \otimes \boldsymbol{e}_{T} .
$$

\subsection{Equilibrium equations}

The equilibrium equations of the equivalent rod model can obtained variationally from the energy formulation of section 2.2. This variational derivation of the equilibrium equations for non-linear elastic rods is classical (SF93, AP10, DA15, PT19). It is a constrained variational problem because of the compatibility equation (2.1) and of the orthonormality condition (2.2). The constraints are handled using the method of Lagrange multipliers.

This variational derivation yields the following set of equilibrium equations:

- kinematic equations, which are obtained by combining equations from section 2.1 with the constraints (2.4),

$$
\begin{gathered}
\boldsymbol{r}^{\prime}(S)=\boldsymbol{d}_{3}(S) \\
\boldsymbol{d}_{i}(S) \cdot \boldsymbol{d}_{j}(S)=\delta_{i j} \\
\boldsymbol{d}_{i}^{\prime}(S)=\boldsymbol{\kappa}(S) \times \boldsymbol{d}_{i}(S) \\
\boldsymbol{\kappa}(S)=\kappa_{2}(S) \boldsymbol{d}_{2}(S)+\kappa_{3}(S) \boldsymbol{d}_{3}(S)
\end{gathered}
$$

- local balance equations for the forces and moments, which are known as the Kirchhoff equations for the equilibrium of thin rods,

$$
\begin{aligned}
\boldsymbol{R}^{\prime}(S)+\boldsymbol{f}(S) & =\mathbf{0} \\
\boldsymbol{M}^{\prime}(S)+\boldsymbol{r}^{\prime}(S) \times \boldsymbol{R}(S) & =0,
\end{aligned}
$$

where $\boldsymbol{f}(S)$ is the external force applied on the rod, per unit length $\mathrm{d} S, \boldsymbol{R}(S)$ is the internal force and $\boldsymbol{M}(S)$ is the internal moment,

- constitutive laws for the internal moment $\boldsymbol{M}(S)=M_{1}(S) \boldsymbol{d}_{1}(S)+M_{2}(S) \boldsymbol{d}_{2}(S)+M_{2}(S) \boldsymbol{d}_{3}(S)$ :

$$
\begin{aligned}
& M_{2}(S)=\frac{\partial W}{\partial \kappa_{2}}\left(\kappa_{2}(S), \kappa_{3}(S)\right) \\
& M_{3}(S)=\frac{\partial W}{\partial \kappa_{3}}\left(\kappa_{2}(S), \kappa_{3}(S)\right),
\end{aligned}
$$

where $W$ is the strain energy density of equation (2.6). Note that the bending moment $M_{1}(S)$ is the Lagrange multiplier associated with the constraint $\kappa_{1}(S)=0$ in equation (2.4) and the internal force $\boldsymbol{R}(S)$ is the Lagrange multiplier associated with the compatibility equation $\boldsymbol{r}^{\prime}(S)=\boldsymbol{d}_{3}(S)$ : neither of them are given by a constitutive law

- boundary conditions, which depend on the particular problem considered.

Overall, this defines a boundary-value problem governing the equilibrium. Its solution is illustrated in sections 4 and 5 in two typical geometries.

\section{Limit CASES}

This section expands on section 2.3: we derive various ribbon models that have been reported in the literature, as limit cases of our model.

\subsection{Small strain: equivalence with linear beam theory}

As shown in equation (2.11), the strain energy (2.6) can be approximated for small strain, $\left|\kappa_{2}\right| \ll \kappa^{\star}$ and $\left|\kappa_{3}\right| \ll \kappa^{\star}$, by the quadratic potential

$$
W\left(\kappa_{2}, \kappa_{3}\right) \approx \frac{Y I_{2}}{2} \kappa_{2}^{2}+\frac{\mu J}{2} \kappa_{3}^{2} \quad \text { (small strain) }
$$

which is plotted as the outer, transparent paraboloid in figure 2.2. Here we have identified $\mu=\frac{Y}{2(1+\nu)}$ as the shear modulus of the material, $I_{2}=\frac{a t^{3}}{12}$ as the geometric moment of inertia, and $J=\frac{a t^{3}}{3}$ as the torsional constant of the slender rectangular cross-section in the limit $t \ll a$. The quadratic approximation of $W$ in equation (3.1) appears to match the prediction from the linear beam theory.

Inserting this expression of $W$ into the general constitutive relations (2.17), we obtain the classical constitutive laws from linear beam theory,

$$
M_{2}=Y I_{2} \kappa_{2} \quad M_{3}=\mu J \kappa_{3} .
$$

Our model is therefore consistent with the linearly elastic (Kirchhoff) theory whenever $\left|\kappa_{2}\right| \ll \kappa^{\star}$ and $\left|\kappa_{3}\right| \ll \kappa^{\star}$. Note that the linearly elastic constitutive relation must be used in conjunction with the constitutive constraints $\varepsilon=0$ and $\kappa_{1}=0$ from equation (2.4).

We now proceed to analyze the small-strain limit of the deflection $w_{\boldsymbol{h}}(T)$ predicted by our non-linear model: in the relevant $q \rightarrow 0$ limit, the deflection in equation (2.13) becomes

$$
w_{\boldsymbol{h}}(T) \approx a^{2} \frac{\nu \kappa_{2}}{2}\left(\frac{1}{12}-\frac{T^{2}}{a^{2}}\right) \quad\left(\left|\kappa_{2}\right| \ll \kappa^{\star}\right) .
$$

This expression has been used to plot the deformed ribbon in figure $2.3 \mathrm{~b}$. 
The deflection in equation (3.3) can be compared to the predictions of the linear beam theory as follows. The deflection $w_{\text {beam }}$ predicted by the linear beam theory is $w_{\text {beam }}(T, Z)=-\nu \varepsilon Z-\nu \kappa_{2} \frac{T^{2}-Z^{2}}{2}$ when $\kappa_{1}=0$, where $Z$ is the coordinate across the thickness, $-\frac{t}{2} \leqslant Z \leqslant+\frac{t}{2}$. Note that the twisting of a linear beam gives rise to a purely longitudinal displacement; this is why $\kappa_{3}$ does not enter into the expression of $w_{\text {beam }}(T, Z)$. Along the mid-surface, $w_{\text {beam }}(T, 0)=-\nu \kappa_{2} \frac{T^{2}}{2}$, and we recover equation (3.3) up to a rigid-body translation-our analysis requires that the average of $w$ is zero on the cross-section but the linear beam theory does not. The displacement predicted by our model in the limit of small strain matches that predicted by the linear beam theory.

Using equation (2.14), we can reconstruct the curvature tensor of the mid-surface as $\boldsymbol{B}=\kappa_{2}\left(\boldsymbol{e}_{S} \otimes \boldsymbol{e}_{S}-\right.$ $\left.\nu \boldsymbol{e}_{T} \otimes \boldsymbol{e}_{T}\right)-\kappa_{3}\left(\boldsymbol{e}_{S} \otimes \boldsymbol{e}_{T}+\boldsymbol{e}_{T} \otimes \boldsymbol{e}_{S}\right)$. The ratio of the longitudinal to the transverse curvature of the mid-surface is therefore $B_{T T} / B_{S S}=-\nu \kappa_{2} / \kappa_{2}=-\nu$, which shows that the cross-section of the ribbon bends in a direction opposite to that of the center-line when $\nu>0$, as can be verified in figure $2.3 \mathrm{~b}$. This effect is known as 'anti-clastic curvature' in the literature on linear beams, and it is fully captured by our model; indeed, based on the linear beam solution we can derive similarly $\frac{1}{\kappa_{2}} \partial^{2} w_{\text {beam }} / \partial T^{2}=-\nu$. Relatedly, note that $\operatorname{det} \boldsymbol{B}_{\boldsymbol{h}}=-\nu \kappa_{2}^{2}-\kappa_{3}^{3}$ is negative when $\nu>0$, i.e., the mid-surface of the ribbon is hyperbolic (saddle-like).

Overall, the strain energy density, the constitutive relations, and the microscopic displacements predicted by our model are fully consistent with those from the linear beam theory in the limit $\left|\kappa_{2}\right| \ll \kappa^{\star}$ and $\left|\kappa_{3}\right| \ll \kappa^{\star}$.

\subsection{Large strain: equivalence with Sadowsky inextensible ribbon model}

In a classical paper, Sadowsky derived the strain energy of an elastic ribbon (Sad29) whose mid-surface is inextensible. An english translation is available (HF15). Inextensible ribbon models have been applied to various geometries such as the elastic Möbius band (SvdH07).

Equation (2.12) shows that the strain energy density (2.6) is equivalent in the limit of large strain, $\left|\kappa_{2}\right| \gg \kappa^{\star}$, to that of Sadowsky. The convergence of the energy is illustrated in figure 2.2, where the inner transparent surfaces made up of two tubes represents Sadowsky's strain energy.

We proceed to check the convergence of the displacement as well. For $\left|\kappa_{2}\right| \gg \kappa^{\star}$, we take the $q \rightarrow \infty$ limit of the displacement predicted by our model in equation (2.13),

$$
w_{\boldsymbol{h}}(T) \approx-a^{2} \frac{\kappa_{3}^{2}}{2 \kappa_{2}}\left(\frac{1}{12}-\frac{T^{2}}{a^{2}}\right) \quad\left(\left|\kappa_{2}\right| \gg \kappa^{\star}\right) .
$$

This expression has been used to draw the helical ribbon in figure 2.3c. From equation (2.14), the curvature tensor associated with the microscopic solution (3.4) is $\boldsymbol{B}=\kappa_{2} \boldsymbol{e}_{S} \otimes \boldsymbol{e}_{S}-\kappa_{3}\left(\boldsymbol{e}_{S} \otimes \boldsymbol{e}_{T}+\boldsymbol{e}_{T} \otimes \boldsymbol{e}_{S}\right)+\frac{\kappa_{3}^{2}}{\kappa_{2}} \boldsymbol{e}_{T} \otimes \boldsymbol{e}_{T}$. The Gauss curvature is obtained as $\operatorname{det} \boldsymbol{B}=\kappa_{2} \frac{\kappa_{3}^{2}}{\kappa_{2}}-\left(-\kappa_{3}\right)^{2}=0$, implying that our microscopic solution is indeed developable in the limit $\left|\kappa_{2}\right| \gg \kappa^{\star}$ : by Gauss' Theorema egregium, this is consistent with the inextensibility assumption of Sadowsky. In addition, we have $\boldsymbol{B} \cdot\left(\kappa_{3} \boldsymbol{e}_{S}+\kappa_{2} \boldsymbol{e}_{T}\right)=0$, which shows that the principal flat direction along the surface is spanned by tangent vector $\kappa_{3} \boldsymbol{d}_{3}+\kappa_{2} \boldsymbol{d}_{2}$. This flat direction makes an angle $\tan ^{-1} \frac{\kappa_{3}}{\kappa_{1}}$ with the tangent $\boldsymbol{d}_{3}$ to the center-line; it is shown by the orange dashed line in figure $2.3 \mathrm{c}$, and is actually a generatrix of the (developable, hence ruled) mid-surface of the ribbon, which is aligned with the axis of the cylinder on which the ribbon is lying. In Sadowsky's analysis, the generatrices make an angle $\tan ^{-1} \frac{\kappa_{3}}{\kappa_{1}}$ with the tangent to the center-line as well.

\subsection{Planar case: equivalence with Shield's model}

In this section, we focus on the pure bending case, by setting $\kappa_{3}=0$. This case has been studied in an early and remarkable paper by Shi92.

From equation (2.6), we have

$$
\begin{aligned}
W\left(\kappa_{2}, 0\right) & \approx \frac{1}{2} \frac{Y a t^{3}}{12} \kappa_{2}^{2}+\frac{1}{2} \frac{Y a^{5} t}{2}\left(\nu \kappa_{2}^{2}\right)^{2} \varphi\left(\frac{\kappa_{2}}{\kappa^{\star}}\right) \\
& \approx \frac{1}{2} \frac{Y a t^{3}}{12\left(1-\nu^{2}\right)} \kappa_{2}^{2}\left(1-\nu^{2}\left[1-\frac{\varphi\left(\frac{\kappa_{2}}{\kappa^{\star}}\right)}{2\left(\frac{\kappa_{2}}{\kappa^{\star}}\right)^{-2}}\right]\right)
\end{aligned}
$$

The square bracket varies between 1 for $\kappa_{2} / \kappa^{\star} \rightarrow 0$ and 0 for $\kappa_{2} / \kappa^{\star} \rightarrow \infty$ (recall that $\varphi(v) \approx 2 v^{-2}$ for large $v$ ). As a result, the bending modulus effectively varies from $\frac{Y a t^{3}}{12}$ for $\kappa_{2} / \kappa^{\star} \rightarrow 0$, in agreement with the prediction of the linear beam theory, to $\frac{Y a t^{3}}{12\left(1-\nu^{2}\right)}$ for $\kappa_{2} / \kappa^{\star} \rightarrow \infty$, in agreement with Sadowsky's inextensible ribbon model. We emphasize that, the plate model predicts the correct bending modulus $\frac{Y a t^{3}}{12}$ for small strain, that agrees with the linear beam model - it is a widespread misconception that the plate model yields the effective bending modulus of a ribbon as $\frac{Y_{a} t^{3}}{12\left(1-\nu^{2}\right)}$ but this assumes implicitly that the plate's mid-surface is cylindrical which is incorrect for small strain (anti-clastic curvature effect).

The non-linear constitutive relation (3.5) shows that the bending modulus varies by a relative amount $\left(\frac{1}{1-\nu^{2}}-1\right)$ between the small strain and the large strain regimes. This relative variation is of order $\sim 15 \%$ for typical values of Poisson's modulus, meaning that the nonlinearity of the constitutive relation has a weak effect in the pure-bending case. Its effect is much more pronounced in the presence of twist, as the analysis of the lateral-torsional instability will reveal in section 4 .

The constitutive law for the bending moment $M_{2}$ is obtained by differentiating equation (3.5) with respect to $\kappa_{2}$ :

$$
M_{2}\left(\kappa_{2}, 0\right)=\frac{Y a t^{3}}{12\left(1-\nu^{2}\right)} \kappa_{2}\left(1-\nu^{2}\left[1-\left(\left(\frac{\kappa_{2}}{\kappa^{\star}}\right)^{2} \varphi\left(\frac{\kappa_{2}}{\kappa^{\star}}\right)+\frac{1}{4}\left(\frac{\kappa_{2}}{\kappa^{\star}}\right)^{3} \varphi^{\prime}\left(\frac{\kappa_{2}}{\kappa^{\star}}\right)\right)\right]\right) .
$$


One can check that this expression is identical to the bending moment derived by Shield in his equations [2.18-2.21] by noticing that Shield's quantities $(b, h, q, 2 D b \kappa, L-K)$ correspond to $\left(\frac{a}{2}, \frac{t}{2}, \frac{1}{a} \sqrt{\frac{\left|\kappa_{2}\right|}{2 \kappa^{\star}}}, \frac{Y a t^{3}}{12\left(1-\nu^{2}\right)}, 1-\right.$ $\left.\left(\bar{\kappa}_{2}^{2} \varphi\left(\bar{\kappa}_{2}\right)+\frac{1}{4} \bar{\kappa}_{2}^{3} \varphi^{\prime}\left(\bar{\kappa}_{2}\right)\right)\right)$, respectively, in our own notation.

\subsection{The case of pure twist}

For $\kappa_{2}=0$, we have from equation (2.6),

$$
W\left(0, \kappa_{3}\right)=\frac{1}{2} \frac{Y a t^{3}}{6(1+\nu)} \kappa_{3}^{2}+\frac{1}{2} \frac{Y a^{5} t}{720} \kappa_{3}^{4} .
$$

The constitutive law for pure twisting can the be obtained from equation (2.17) as

$$
M_{3}\left(0, \kappa_{3}\right)=\frac{Y a t^{3}}{6(1+\nu)} \kappa_{3}+\frac{Y a^{5} t}{360} \kappa_{3}^{3} .
$$

When $t \ll a$, this matches the result obtained by Green (Gre36, Gre37), see for example equation 6 in reference (Gre37); note that $(a, t, Y)$ in our notation corresponds to $(2 b, 2 h, 2 \mu(1+\nu))$ in his notation.

\subsection{A simple approximation for $\varphi$, comparison to the work of Sano et al.}

Inspired by the work of GB05, SW19, we consider a simple approximation to the function $\varphi(v)$ from equation (2.8),

$$
\varphi_{\mathrm{Sano}}(v)=\frac{1}{360+\frac{v^{2}}{2}} .
$$

By design, it is consistent with the original function $\varphi$ in both the small-strain and large-strain limits, as $\varphi$ Sano $(0)=$ $\frac{1}{360}=\varphi(0)$ and $\varphi_{\text {Sano }}(v) \sim \frac{2}{v^{2}} \sim \varphi(v)$ for $v \rightarrow \pm \infty$. The original and approximate functions $\varphi$ and $\varphi$ Sano and are plotted in figure 2.2: the approximation $\varphi \simeq \varphi_{\text {Sano }}$ appears to be acceptable but not very accurate; it is still expected to perform quite well as the factor $\varphi$ enters only in the correction to the strain energy beyond the quadratic order, see equation (2.6).

Inserting $\varphi \simeq \varphi_{\text {Sano }}$ into the ribbon energy from equation (2.6), we obtain the approximate strain energy

$$
W\left(\kappa_{2}, \kappa_{3}\right) \simeq \frac{1}{2} \frac{Y a t^{3}}{12} \kappa_{2}^{2}+\frac{1}{2} \frac{Y a t^{3}}{6(1+\nu)} \kappa_{3}^{2}+\frac{1}{2} \frac{Y a t^{3}}{12\left(1-\nu^{2}\right)} \frac{\left(\nu \kappa_{2}^{2}+\kappa_{3}^{2}\right)^{2}}{\frac{60}{1-\nu^{2}} \frac{t^{2}}{a^{4}}+\kappa_{2}^{2}}
$$

It can be used to set up simulations without having to cope with the original function $\varphi$, whose numerical evaluation requires more work.

The ribbon energy introduced by SW19 based on heuristic arguments matches that in equation (3.7) in the case $\nu=0$

\section{LATERAL-TORSIONAL BUCKLING}

The lateral-torsional buckling of a beam having a rectangular cross-section is a classical problem in elasticity (Mic99). In this section, we study the case sketched in figure 4.1 where the aspect-ratio $a / t$ of the cross-section is large: we use our one-dimensional ribbon model to analyze the instability. Its predictions are confronted to experiments and to finite-element simulations of a two-dimensional shell model.

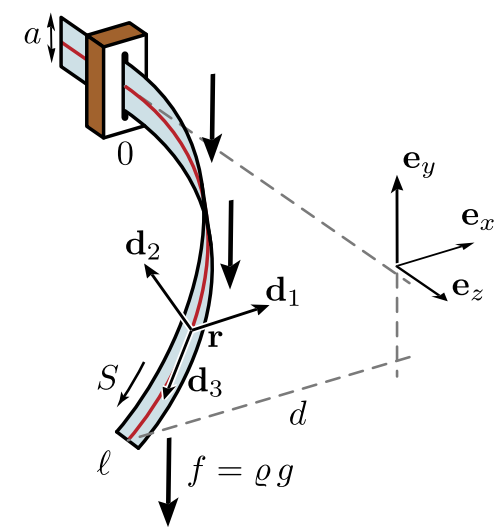

Figure 4.1. Lateral-torsional buckling of a ribbon: typical buckled configuration. The buckling amplitude is measured by the horizontal deflection $d=\left|\boldsymbol{r}(\ell) \cdot \boldsymbol{e}_{x}\right|$ of the endpoint. 


\subsection{Problem formulation}

A ribbon with initial length $\ell$ is clamped at $S=0$, in such a way that both its initial normal $\boldsymbol{d}_{1}(0)$ and tangent $\boldsymbol{d}_{3}(0)$ at the clamp are horizontal. The ribbon is subjected to its own weight, which is represented in the model by a distributed force with density $f=\rho$ at $g=\varrho g$ per unit length $\mathrm{d} S$. Here $\rho$ is the mass per unit volume of the material, $a$ and $t$ are the ribbon width and thickness, as earlier, $\varrho=\rho a t$ is the mass per unit length of the ribbon, and $g$ the acceleration of gravity. The other endpoint $S=\ell$ of the ribbon is stress-free.

For a sufficiently short length $\ell$, the only equilibrium solution is the straight, untwisted one. For larger values of $\ell$, a new family of solutions with both twisting and bending appears, as sketched in figure 4.1. It is produced by the so-called lateral-torsional buckling instability: for sufficiently long ribbons, the elastic energy in the buckled (twisted and bent) configuration is compensated by the decrease in the potential gravitational energy. In the literature, the lateral-torsional buckling instability has been studied using the linearly elastic rod model from equations (2.11) or (3.2). It is analyzed here based on the more general non-linear model established in section 2, which takes into account the stretchability of the mid-surface.

This problem can be analyzed mathematically using $(i)$ the clamping conditions at $S=0$,

$$
\boldsymbol{r}(0)=\mathbf{0} \quad \boldsymbol{d}_{i}(0)= \begin{cases}\boldsymbol{e}_{y} & \text { if } i=1 \\ \boldsymbol{e}_{z} & \text { if } i=2 \\ \boldsymbol{e}_{x} & \text { if } i=3\end{cases}
$$

(ii) the stress-free boundary conditions at $S=\ell$,

$$
\boldsymbol{R}(\ell)=\mathbf{0} \quad \boldsymbol{M}(\ell)=0
$$

(iii) the kinematic equations (2.1-2.4), which can be combined as follows

$$
\boldsymbol{r}^{\prime}(S)=\boldsymbol{d}_{3}(S) \quad \boldsymbol{d}_{i}(S) \cdot \boldsymbol{d}_{j}(S)=\delta_{i j} \quad \boldsymbol{d}_{i}^{\prime}(S)=\left(\omega_{2} \boldsymbol{d}_{2}(S)+\omega_{3}(S) \boldsymbol{d}_{3}(S)\right) \times \boldsymbol{d}_{i}(S),
$$

(iv) the local equilibrium equations (2.16) with $\boldsymbol{f}=-\varrho g \boldsymbol{e}_{y}$, where $\boldsymbol{e}_{y}$ is a unit vertical vector pointing upwards, as well as $(v)$ the decomposition of the internal moment $\boldsymbol{M}(S)=M_{1}(S) \boldsymbol{d}_{1}(S)+M_{2}(S) \boldsymbol{d}_{2}(S)+M_{2}(S) \boldsymbol{d}_{3}(S)$ and the constitutive laws (2.17).

These equations form a non-linear one-dimensional boundary-value problem, which we solve numerically for various values of the loading and geometric parameters. For comparison purposes, these numerical results are not only produced using the extensible ribbon model, obtained by inserting the strain energy functionals (2.6) into the constitutive laws (2.17), but also using the linearly elastic model as well as Sadowsky's inextensible ribbon model, obtained by using the strain energy functionals (3.1) or (2.12), respectively.

Dimensional analysis shows that the solution to the extensible ribbon model depends on 3 dimensionless parameters

$$
\left(\nu, \ell \kappa^{\star}, \gamma\right)
$$

where $\nu$ is Poisson's ratio, $\gamma$ is the dimensionless weight,

$$
\gamma=\frac{\varrho g \ell^{3}}{Y a t^{3} / 12}
$$

and $\ell \kappa^{\star}$ is a geometric parameter, see equation (2.7). The parameter $\ell \kappa^{\star}$ can be rewritten in several ways, such as

$$
\begin{aligned}
\ell \kappa^{\star} & =\frac{1}{\left[12\left(1-\nu^{2}\right)\right]^{1 / 2}} \frac{\ell t}{a^{2}} \\
& =\frac{t}{t^{\star}} \quad \text { where } t^{\star}=\frac{\left[12\left(1-\nu^{2}\right)\right]^{1 / 2} a^{2}}{\ell} .
\end{aligned}
$$

Here, we have introduced a typical thickness $t^{\star} \sim a^{2} / \ell$ depending on the width $a$ and length $\ell$ of the ribbon.

\subsection{Linear bifurcation analysis}

We start by deriving the critical value of the dimensionless buckling load $\gamma_{\mathrm{c}}$ by a linear bifurcation analysis. The detailed analysis is presented in appendices $\mathrm{C}$ and $\mathrm{D}$ and its results can be summarized as follows. The buckling load $\gamma_{\mathrm{c}}$ predicted by the extensible ribbon model $(2.6)$ is $\gamma_{\mathrm{c}}=\frac{18.178}{\sqrt{1+\nu}}$. The buckling load $\gamma_{\mathrm{c}}^{\text {lin }}$ predicted by the linearly elastic model (3.1) is identical but, surprisingly, the buckling load $\gamma_{\mathrm{c}}^{\text {sdw }}$ predicted by Sadowsky's inextensible model is different:

$$
\gamma_{\mathrm{c}}=\gamma_{\mathrm{c}}^{\text {lin }}=\frac{18.178}{\sqrt{1+\nu}} \quad \gamma_{\mathrm{c}}^{\mathrm{sdw}}=\frac{21.491}{1-\nu^{2}}
$$

In figure 4.2, these critical loads have been evaluated for $\nu=0.4$, and placed on the $\gamma$-axis. The buckling load predicted by Sadowsky's model appears to be significantly larger than the two other ones. 


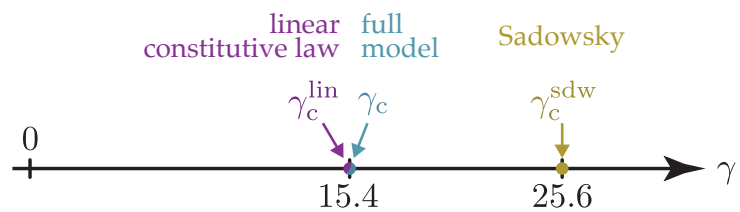

Figure 4.2. First critical buckling load for the lateral-torsional buckling, as predicted by the different models, for $\nu=0.4$, from equation (4.4). Note that the predictions from the extensible ribbon model (section 2) coincides with that from the linearly elastic model, and that Sadowsky's model predicts a buckling load that is $66 \%$ larger for this particular value of $\nu$.

a)

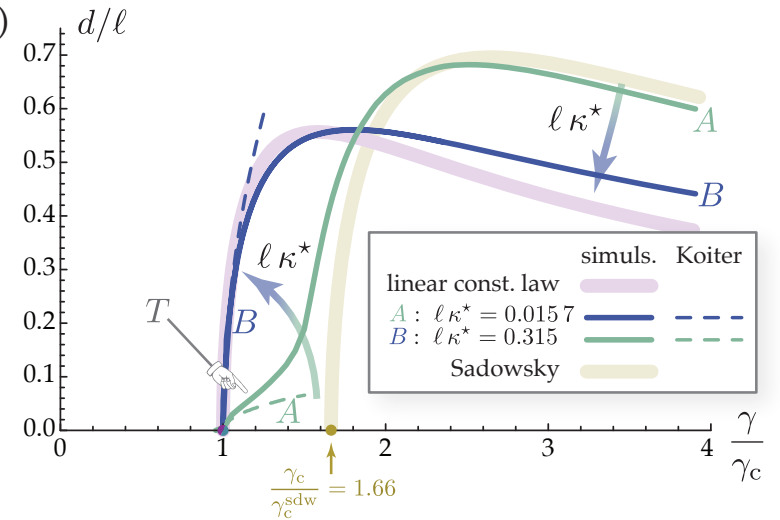

b)

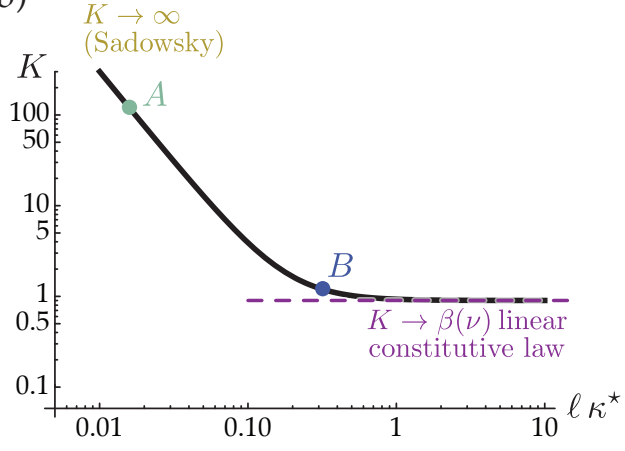

Figure 4.3. (a) Numerical solutions to the lateral-torsional buckling problem, obtained using AUTO-07p for $\nu=0.4$ The scaled lateral deflection $d / \ell=\left|\boldsymbol{r}(\ell) \cdot \boldsymbol{e}_{x}\right| / \ell$ is plotted as a function of the dimensionless load parameter $\gamma / \gamma_{\mathrm{c}}$. The thin curves are the predictions of the extensible ribbon model, for a thinner ribbon $\left(A, \ell \kappa^{\star}=0.0157\right)$ and a thicker ribbon $\left(B, \ell \kappa^{\star}=0.315\right)$. The dashed half-parabolas are Koiter's weakly non-linear predictions from equation (4.5). For thin enough ribbons such as $A$, the initial buckling regime produces a tongue $T$ in the bifurcation diagram. The thick, light curves are the predictions of the linearly elastic rod model and of Sadowsky's inextensible ribbon model. (b) Log-log plot of the initial curvature $K$ predicted by Koiter's weakly post-buckling analysis, as a function of the parameter $\ell \kappa^{\star}$

We have shown in section 3 that Sadowsky's inextensible model can be recovered from the extensible ribbon model by letting the thickness go to zero, $t \rightarrow 0$. This seems in conflict with the existence of a fixed (and significant) gap between the critical loads $\gamma_{\mathrm{c}}$ and $\gamma_{\mathrm{c}}^{\mathrm{sdw}}$ predicted by these two models. With the aim to clarify why the critical load does not pass to the limit for $t \rightarrow 0$, we proceed to analyze the post-buckled solutions of the lateral-torsional instability.

\subsection{Non-linear solutions}

We solve the non-linear boundary-value problem (BVP) from section 4.1 using the numerical library AUT0-07p, which handles one-dimensional non-linear BVP's using a continuation method (DKK91); the implementation of a similar one-dimensional problem using AUT0-07p is discussed by MGN18. The numerical evaluation of the function $\varphi$ appearing in equation (2.8) is documented in appendix B.

Typical numerical solutions are shown in figure 4.3a. The thin curves labelled $A$ and $B$ show the scaled horizontal deflection $d / \ell=\left|\boldsymbol{r}(\ell) \cdot \boldsymbol{e}_{x}\right| / \ell$ of the free end of the ribbon as a function of the scaled load parameter $\gamma / \gamma_{\mathrm{c}}$. Both curves emerge from the pitchfork bifurcation point located at the same value $\gamma=\gamma_{\mathrm{c}}$ of the load, thereby confirming the results of the linear bifurcation analysis from section 4.2. The two curves were generated using the same value of Poisson's ratio $\nu=0.4$, but different values of the parameter $\ell \kappa^{\star}$, which is 0.0157 for $A$ and 0.315 for $B$. As $\ell \kappa^{\star}=t / t^{\star}$, see equation (4.3), we will refer to $A$, which has the smaller value of $\ell \kappa^{\star}=t / t^{\star}$, as the thinner ribbon, and to $B$ as the thicker ribbon. The ribbon $B$ is called thick by a slight abuse of language, to express the fact that its thickness $t$ is of order $t^{\star}$ (and not much smaller than $t^{\star}$ ); given that $t^{\star} \ll a$, it still satisfies $t \ll a$, as assumed everywhere in this paper.

In figure 4.3a, the bifurcation diagram shows the post-buckling behavior of both ribbons when the load $\gamma$ is increased. Unlike the thicker ribbon $B$ which follows a smooth curve, the thinner ribbon $A$ produces a 'tongue', denoted as $T$ in the diagram, with a large initial curvature in the $\left(\gamma / \gamma_{c}, d / \ell\right)$ plane, followed by a smoother regime. For comparison, the curves predicted by the linearly elastic model (thick, light purple curve) and by Sadowsky model (thick, light brown curve) have been included in the diagram. The results are generally consistent, in the sense that the thicker ribbon $B$ follows closely the prediction of the linearly elastic model for moderate values of the load, while the thinner ribbon $A$ follows closely the prediction of Sadowsky model for larger values of the load.

Interestingly the post-buckling curve of the thinner ribbon $A$ displays two regimes. In the immediate vicinity of the buckling load $\gamma_{\mathrm{c}}=\gamma_{\mathrm{c}}^{\text {lin }}$, the post-buckled branch is close to that predicted by the linearly elastic model: this is because deflection and the strain are initially very small. Further down on the bifurcation branch, however, the branch follows the prediction of Sadowsky model very closely: this happens for larger strain values. These two regimes a connected by the tongue $T$. 
To provide further insights, we have calculated the initial curvature of the branch in the bifurcation diagram used the weakly non-linear Koiter expansion method (calculations not shown). The result is a parabolic approximation of the bifurcated branch near the bifurcation point,

$$
\frac{d}{\ell} \approx \pm \sqrt{\frac{2}{K}}\left(\frac{\gamma}{\gamma_{\mathrm{c}}}-1\right)^{1 / 2} \quad \text { (Koiter post-buckling expansion) }
$$

where the initial curvature $K$ is given by

$$
K=\frac{\alpha(\nu)}{\left(\ell \kappa^{\star}\right)^{2}}+\beta(\nu) \text { where }\left\{\begin{array}{l}
\alpha(\nu)=\frac{0.009906+0.03302 \nu+0.04315 \nu^{2}}{\left(1-\nu^{2}\right)} \\
\beta(\nu)=\frac{0.9658+0.2680 \nu-0.9658 \nu^{2}-0.2680 \nu^{3}}{\left(1-\nu^{2}\right)}
\end{array}\right.
$$

The prediction (4.5) from the Koiter expansion is plotted as dashed half-parabolas in figure 4.3a; it correctly captures the initial curvatures of both solution branches. The curvature $K$ is plotted in figure $4.3 \mathrm{~b}$ as a function of the parameter $\ell \kappa^{\star}$ : for very thin ribbons $\left(\ell \kappa^{\star} \rightarrow 0\right), K$ goes to infinity as $K \sim \frac{\alpha(\nu)}{\left(\ell \kappa^{\star}\right)^{2}}$, implying that the tongue in the bifurcation diagram becomes very sharp. In addition, the initial curvature $K$ appears to be a decreasing function of $\ell \kappa^{\star}$. This suggests the following evolution of the curves in the bifurcation diagram when $\ell \kappa^{\star}$ is varied, as depicted by the colored arrows in figure 4.3a: for very small values of $\ell \kappa^{\star}$ (i.e., for $t \ll t^{\star}$ ) the bifurcation curve is a sharp tongue emanating from the bifurcation point at $\gamma / \gamma_{c}=1$, that connects with the prediction of Sadowsky model around $\gamma / \gamma_{\mathrm{c}}=\gamma_{\mathrm{c}}^{\mathrm{sdw}} / \gamma_{\mathrm{c}}=1$.66. As $\ell \kappa^{\star}$ gets larger, the initial curvature $K$ decreases, the tongue gets thicker, and the bifurcation curve sits half-way between the predictions of the linearly elastic model and Sadowsky model. For values of $\ell \kappa^{\star}$ as large as $\sim 1$, the tongue merges into the rest of the branch and the bifurcation curve gets uniformly close to that predicted by the linearly elastic model, as happens with the ribbon $B$.

For very thin ribbons $\left(\ell \kappa^{\star} \ll 1\right)$, the typical width $\tilde{d} / \ell$ of the sharp tongue can be estimated by calculating the intersection of Koiter's parabola, equation (4.5), with the vertical line $\gamma=\gamma_{\mathrm{c}}^{\mathrm{sdw}}$ where the Sadowsky model bifurcates. Using an order of magnitude reasoning, $\gamma_{\mathrm{c}}^{\mathrm{sdw}} / \gamma_{\mathrm{c}}-1=\mathcal{O}(1)$ and equations (4.5-4.6) yield $\tilde{d} / \ell \sim K^{-1 / 2} \sim \ell \kappa^{\star}$. No matter how small the parameter $\ell \kappa^{\star}$ is, a very thin ribbon always bifurcates like a linearly elastic rod initially, when the deflection is small enough $\left(d / \ell \ll \tilde{d} / \ell \sim \ell \kappa^{\star}\right.$, tongue tip), and it is only for larger deflections $\left(d / \ell \gg \tilde{d} / \ell \sim \ell \kappa^{\star}\right)$ that the Sadowsky approximation becomes applicable. This shows that the convergence to Sadowsky model is nonuniform when the ribbon thickness is decreased $\left(\ell \kappa^{\star} \rightarrow 0\right)$. This non-uniform convergence explains why the buckling threshold predicted by Sadowsky model does not match those of the two other models, see equation (4.4): for a ribbon with non-zero thickness, Sadowsky's model is never applicable in the early post-buckled range.

\subsection{Comparison to experiments and to shell simulations}

We carried out a series of experiments using thin ribbons cut out of overhead-projector sheets made of cellulose acetate having length $297 \mathrm{~mm}$, and thickness $t=0.0925 \mathrm{~mm}$. We used three different ribbons in the experiments, with width $a \in\{1.88,5.10,20.05\} \mathrm{mm}$. The slit mechanism shown in figure 4.1 is achieved in the experiments by blocking the ribbon between two cubes pressed against each other using a clamp; by releasing the clamp and sliding the ribbon against the cubes, we could vary the overhanging length $\ell$ continuously between 0 and 270 mm. To minimize the effect of the non-planarity of the acetate sheets, the horizontal deflection $d$ of the two symmetrically buckled configurations has been measured and the average value has been used. By varying the overhang length $\ell$, we obtained three series of values for $(\ell, d)$, one for each of the three possible ribbon widths $a$.

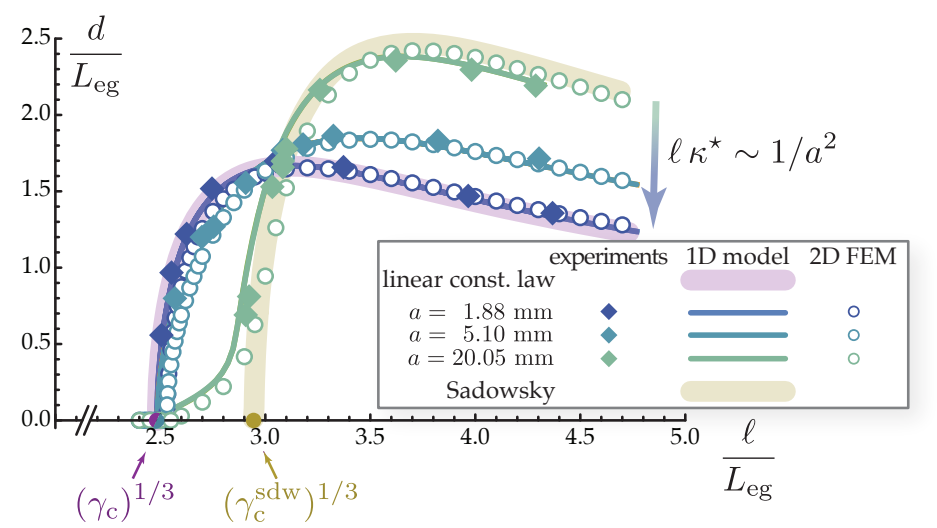

Figure 4.4. Comparison of the bifurcation diagrams for the lateral-torsional buckling of three ribbons having different widths, with thickness $t=0.0925 \mathrm{~mm}$ : experiments using a cellulose acetate sheet (filled diamonds), numerical simulations of the one-dimensional model using AUT0-07p (thin curves), and finite-element simulations of the Naghdi shell model (open disks). The simulations of both the one-dimensional rod model and of the twodimensional shell model use a Poisson's ratio $\nu=0.4$, which is typical of the material used in the experiments. A single parameter, $L_{\mathrm{eg}}=62.3 \mathrm{~mm}$, has been adjusted. 
To compare the experiments with the predictions of the one-dimensional model, we used the same numerical method as in section 4.3. In the simulations, we set the Poisson's ratio to $\nu=0.4$, which is typical of the cellulose acetate material used in the experiments, and we set the geometrical parameters $t$ and $a$ to their values in the experiments. In the list (4.1) of dimensionless parameters, the parameters $\nu$ and $\ell \kappa^{\star}$ are therefore known for any value of the overhang $\ell$, but the loading parameter $\gamma$ from equation (4.2) is not, because the experimental value of the Young modulus $Y$ is not known so far. We rewrite it as $\gamma=\left(\ell / L_{\mathrm{eg}}\right)^{3}$ where $L_{\mathrm{eg}}$ is the elasto-gravitational length

$$
L_{\mathrm{eg}}=\left(\frac{Y a t^{3} / 12}{\varrho g}\right)^{1 / 3}=\left(\frac{Y t^{2} / 12}{\rho g}\right)^{1 / 3}
$$

and recall that $\rho$ is the mass density of the material per unit volume while $\varrho=\rho a t$ is the mass density of the ribbon per unit length. The quantity $L_{\text {eg }}$ being independent of the width $a$, it is identical for all three ribbons: we use this quantity $L_{\mathrm{eg}}$ as a fitting parameter in the simulations, i.e., we generate different sets of numerical curves corresponding to different values of $\gamma=\left(\ell / L_{\mathrm{eg}}\right)^{3}$, and choose the value of $L_{\mathrm{eg}}$ that achieves the best agreement overall with the experimental datapoints. Effectively, this amounts to extract the value of the Young modulus $Y$ of the material from the experimental data. The optimal value is $L_{\mathrm{eg}}=62.3 \mathrm{~mm}$; using equation (4.7), this corresponds to a sound wave in the material $\sqrt{Y / \rho}=\left(L_{\mathrm{eg}}^{3} 12 \mathrm{~g} / \mathrm{t}^{2}\right)^{1 / 2}=1820 \mathrm{~m} \cdot \mathrm{s}^{-1}$, which is indeed typical for plastic materials. The result is shown in figure 4.4: filled diamonds correspond to experimental values, and thin curves to numerical results of the one-dimensional model.

In addition, we ran numerical finite-element simulations of the Naghdi thin shell model, using the same set of values of $t, a, \nu$ and $L_{\mathrm{eg}}$ used in the simulations of the one-dimensional model. The Naghdi model is a geometrically exact non-linear shell model comprising bending, extension, and transverse shear deformations (Nag63), and the numerical scheme uses reduced integration to cope with shear-locking behavior (HBBM18). The simulation code is based on the FEniCS-Shells python $/ C^{++}$mixed library described in (HBBM18). The numerical data produced by the finite-element shell simulations have been included in figure 4.4 (open disks) without any additional fitting parameter.

In figure 4.4, an excellent agreement is obtained between the experiments, the one-dimensional model and the twodimensional model. The evolution of the bifurcation curves with the parameter $\ell \kappa^{\star} \sim 1 / a^{2}$, as discussed earlier in section 4.3 based on the one-dimensional model, is fully confirmed. In particular, the presence of a tongue emanating from the bifurcation point is clearly visible in the data produced by the shell model for the widest ribbon (green circles, largest value of $a$, smallest value of $\ell \kappa^{\star}$ ). The consistency with $(i)$ the linearly elastic rod model (thick, light purple curve) for thicker ribbons, and (ii) with Sadowsky model (thick, light brown curve) for thinner ribbons, away from the tongue region, is also confirmed.

\subsection{Discussion}

The bifurcation diagram in figure 4.4 shows that the predictions of the linearly elastic and Sadowsky models are markedly different, and confirms that the extensible ribbon model bridges the gap between them. The excellent agreement between its predictions, the experimental measurements and the finite-element shell simulations confirms that the extensible model accurately captures the mechanical response of ribbons, even in a range of widths $(2 \mathrm{~mm}<a<20 \mathrm{~mm}$ for this particular experiment) that is not covered by the other models. The extensible model ignores the dependence of the energy on the strain gradients $\kappa_{2}^{\prime}(S)$ and $\kappa_{3}^{\prime}(S)$ : in the experiments, the separation $a \ll \ell$ between the longitudinal and transverse scales appears to be sufficient for the effect of the strain gradient to remain negligible.

Sadowsky's inextensible ribbon model is known to be valid in the limit $a / t \rightarrow \infty$, for thin (and wide) ribbons. Now, the strip with intermediate width $a=5.10 \mathrm{~mm}$ used in the experiments features a cross-sectional aspect-ratio $a / t=55.2$, a number that is significantly larger than 1 . One could therefore have expected that this strip would be accurately represented by Sadowsky's model. The bifurcation diagram in figure 4.4 shows that this is far from being the case (intermediate turquoise curve). This apparent paradox is resolved by recognizing that the condition of validity of Sadowsky's model is not $a / t \gg 1$ but instead $|\bar{\kappa}| \gg 1$, where $\bar{\kappa} \sim \kappa / \kappa^{\star} \sim \kappa a^{2} / t$ from equation (1.1), as discussed in the introduction. The statement that Sadowsky's model holds in the limit $a / t \rightarrow \infty$ is correct but it does not imply that its domain of validity is $a / t \gg 1$. Its domain of validity, $|\bar{\kappa}| \gg 1$, actually writes as $a / t \gg 1 /(\kappa a)$ where the lower bound $1 /(\kappa a) \sim \ell / a$ is a large number $(\ell / a \sim 50$ in the present experiments).

We have also studied the Euler buckling of a thin ribbon clamped at one end, and subjected to an axial compressive force at the other. For the sake of brevity, the detailed results are not included but the main findings are shortly discussed here; they are strikingly similar to those for the lateral-torsional buckling instability. In particular the bifurcation diagram resembles that in figure 4.4. The linearly elastic model and the Sadowsky model predict different Euler buckling loads, as well as different bifurcation curves. When the ribbon thickness is held constant, the bifurcation curve predicted by the extensible model goes smoothly from that predicted by the linearly elastic model at small width, to that predicted by Sadowsky model at large width. The convergence to Sadowsky model is not uniform as the linearly elastic model is always applicable sufficient close to bifurcation: the convergence takes places through a tongue region that becomes sharper and sharper as the ribbon width increases; it connects the bifurcation point predicted by the linearly elastic model to the bifurcation branch predicted by Sadowsky's model. A Koiter analysis confirms that the initial curvature of the bifurcation curve for Euler buckling diverges in the limit $\ell \kappa^{\star} \rightarrow 0$, as earlier in equation (4.6) for the lateral-torsional instability. The discrepancy between the linearly elastic model and Sadowsky model, however, turns out to be significantly less for Euler buckling than for the lateral-torsional instability: in the absence of twisting, it essentially arises from a mismatch in the definitions of the bending modulus, which is $Y I_{2}$ in the linearly elastic model from equation (3.2), versus $\frac{Y I_{2}}{1-\nu^{2}}$ in Sadowsky model from equation (3.5), as discussed in section 3.3: the discrepancy is by about $20 \%$, much less than the $65 \%$ mismatch seen in figure 4.2 for the lateral-torsional instability. 
a)

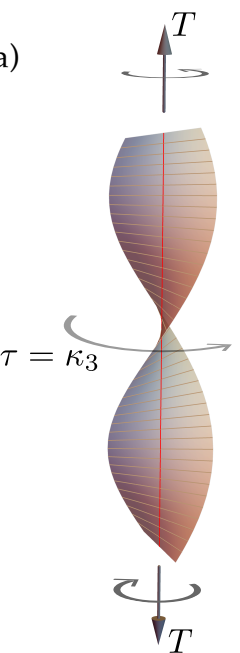

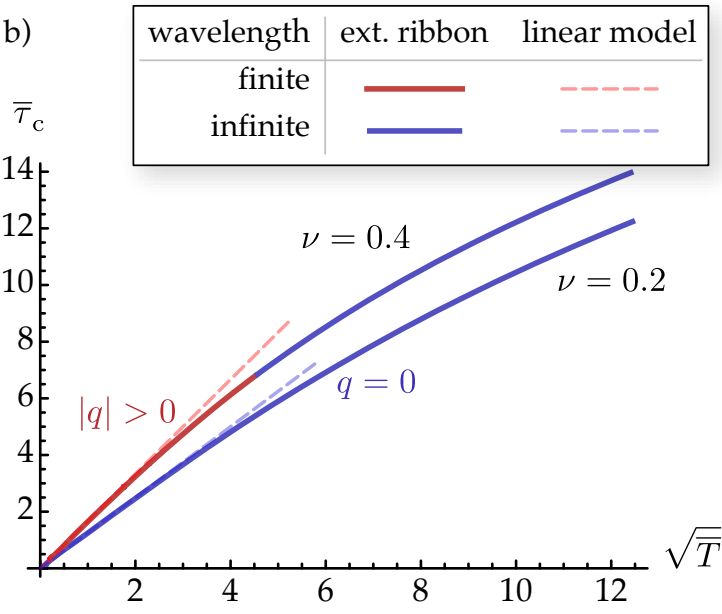

Figure 5.1. Linear bifurcation analysis of a twisted ribbon having infinite length. (a) Geometry. (b) Scaled first critical twisting strain $\bar{\tau}_{\mathrm{c}}=\tau_{\mathrm{c}} / \kappa^{\star}$ as a function of the square root of the scaled applied tension $\bar{T}=T / W^{\star}$; see equations (2.7) and (2.10) for a definition of the scales $\kappa^{\star}$ and $W^{\star}$. The solid curves are the predictions of our ribbon model from equations (5.1-5.3); the dashed lines are the predictions of the small-tension approximation from equation (5.4). Note that the instability switches from finite-wavelength (red, $|q|>0$ ) for larger values of $\nu$ and for moderate applied tension, to infinite-wavelength (blue, $q=0$ ) for smaller values of $\nu$ or larger applied tension.

\section{BUCKLING OF A TWISTED RIBBON UNDER TENSION}

The instability of a twisted rod under tension is another classical instability, which has been extensively studied for circular cross-sections (Gre83). Here, we analyze the buckling of an infinitely long twisted ribbon under tension using our one-dimensional model.

Instabilities of twisted rectangular plates have been analyzed but with a focus on 'microscopic' buckling modes (see figure 1 of CB86 or figure 1c of CK13), having a longitudinal wavelength comparable to the width $a$ (Gre37, CB86, CK13, Moc01). In the present work, the emphasis is instead on a 'macroscopic' buckling mode (see e.g. figure 3.11 of AP10), whose longitudinal wavelength is of order $\left(Y a t^{3} / T\right)^{1 / 2}$, where $T$ is the applied tension, see equation (E.10). This 'macroscopic' instability has not yet been characterized for ribbons. It is in competition with the 'microscopic' one and appears first for sufficiently low traction forces $T$, as we will show.

\subsection{Critical twisting strain}

We derive the linear bifurcation criterion for an infinitely long, twisted ribbon subject to a tension $T$. We denote by $\tau=\kappa_{3}$ the twisting strain in the base configuration.

As shown in appendix E, the critical value $\bar{\tau}_{\mathrm{C}}$ of the scaled twisting strain $\bar{\tau}=\tau / \kappa^{\star}$ is the solution $\bar{\tau}_{\mathrm{C}}=\bar{\tau}$ of the implicit equation

$$
f(t(\bar{T}, \bar{\tau}), m(\bar{T}, \bar{\tau}))=0,
$$

where $f(t, m)$ is the numerical function

$$
f(t, m)=\left\{\begin{array}{lll}
2-\frac{1}{t}\left(\frac{1+m}{2}\right)^{2} & \text { if } 2 t-m \geqslant 1 \quad \text { (finite wavelength) } \\
1-(m-t) & \text { if } 2 t-m<1 \text { (infinite wavelength) }
\end{array}\right.
$$

The arguments of $f$ are the scaled incremental bending modulus $t$ and the scaled twisting moment $m$. They are given in terms of the scaled tension $\bar{T}=\frac{T}{W^{\star}}$ and of $\bar{\tau}$ as

$$
\begin{aligned}
t(\bar{T}, \bar{\tau}) & =\frac{\bar{\tau}^{2}}{\bar{T}}\left(\left(1-\nu^{2}\right)+\frac{\nu}{360} \bar{\tau}^{2}-\frac{1}{2 \cdot 181440} \bar{\tau}^{4}\right) \\
m(\bar{T}, \bar{\tau}) & =\frac{\bar{\tau}^{2}}{\bar{T}}\left(2(1-\nu)+\frac{1}{360} \bar{\tau}^{2}\right) .
\end{aligned}
$$

These equations have been used to generate the curves in figure $5.1 \mathrm{~b}$, showing the scaled critical twisting strain $\bar{\tau}_{\mathrm{C}}$ as a function of the square root of the scaled tension $\bar{T}=T / W^{\star}$, where $W^{\star}$ has been defined in equation $(2.10)$.

The alternatives in the right-hand side of equation (5.2) correspond to the cases where the buckling mode has a finite (top) versus an infinite (bottom) wavelength, as measured in the 'helical' frame attached to the twisted ribbon. The bifurcation curves show a transition from finite to infinite wavelength as the dimensionless tension $\bar{T}=T / W^{\star}$ is increased, when Poisson's ratio is sufficient large (composite red-blue curve corresponding to $\nu=0.4$ in the figure). 


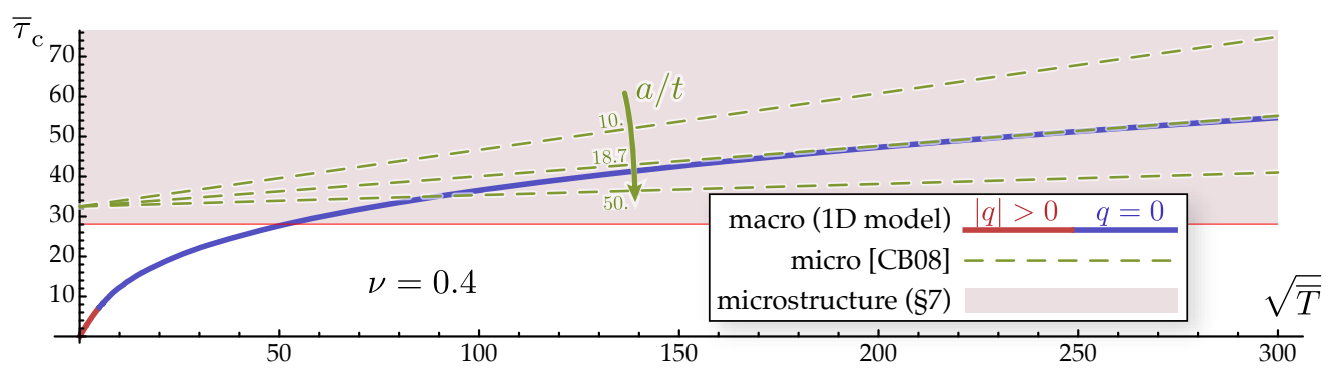

Figure 5.2. Twisted ribbon under tension: competition between the long-wavelength instability (described by the one-dimensional model, thick curves), the short-wavelength instability from CB08 (dashed lines, from equation (5.6)), and the formation of a microstructure (based on equation (7.7), shaded domain). The axes are those of figure 5.1, and the thick curve is the the upper curve of figure 5.1.

\subsection{Limit of a small applied tension}

It is interesting to analyze the case of a small applied tension, $\bar{T} \ll 1$. The critical strain is then small as well, $\left|\bar{\tau}_{\mathrm{c}}\right| \ll 1$, and the ribbon is effectively linearly elastic. One can then ignore the quadratic and quartic terms that appear inside the parentheses in equation (5.3): $t \approx \frac{\bar{\tau}^{2}}{\bar{T}}\left(1-\nu^{2}\right)$ and $m \approx 2(1-\nu) \frac{\bar{\tau}^{2}}{\bar{T}}$. Assuming an infinite-wavelength mode first, the critical load is obtained by solving $0=f=1-(m-t)=1-\frac{((1-\nu) \bar{\tau})^{2}}{\bar{T}}$; the solution is $\bar{\tau}_{\mathrm{C}}=\frac{\sqrt{\bar{T}}}{1-\nu}$. For the assumption of infinite wavelength to be consistent, we must have $0<1-2 t+m=1-\frac{\bar{\tau}^{2}}{\bar{T}} 2 \nu(1-\nu)=1-\frac{2 \nu(1-\nu)}{(1-\nu)^{2}}=\frac{3(1 / 3-\nu)}{(1-\nu)}$ : the wavelength is indeed infinite only as long as $\nu<1 / 3$. The finite-wavelength case can analyzed similarly, and the final result is

$$
\bar{\tau}_{\mathrm{c}}=\left\{\begin{array}{lll}
\sqrt{\bar{T}}\left(\frac{1+(2 \nu+2 \sqrt{\nu(\nu+1)})}{2-2 \nu}\right)^{1 / 2} & \text { if } \nu>1 / 3 & \text { (finite wavelength) } \\
\frac{\sqrt{\bar{T}}}{1-\nu} & \text { if } \nu<1 / 3 & \text { (infinite wavelength) }
\end{array} \quad \text { (small- } \bar{T}\right. \text { limit) }
$$

This equation has been used to generate the dashed lines in figure 5.1b. They correctly capture the small-tension behavior of the complete bifurcation curves.

This critical twisting strain $\bar{\tau}_{\mathrm{c}}$ can be converted into a critical twisting moment $\left(M_{3}\right)_{\mathrm{c}}$ by using the constitutive relation from the linear theory (3.2):

$$
\left(M_{3}\right)_{\mathrm{c}}=\left\{\begin{array}{lll}
\sqrt{\left(Y I_{2}\right) T}\left(2 \frac{1+(2 \nu+2 \sqrt{\nu(\nu+1)})}{1+\nu}\right)^{1 / 2} & \text { if } \nu>1 / 3 & \text { (finite wavelength) } \\
\frac{1}{\sqrt{1-\nu^{2}}} \sqrt{4\left(Y I_{2}\right) T} & \text { if } \nu<1 / 3 & \text { (infinite wavelength) }
\end{array} \quad \text { (small } \bar{T}\right. \text { limit) }
$$

Both these values are slightly larger than the classical prediction $\sqrt{4\left(Y I_{2}\right) T}$ of the linear theory for rods with isotropic cross-sections (Gre83, TG61, Coy90). This increase in the buckling load is consistent with the fact that a ribbon is stiffer than an isotropic rod as it has one finite and one infinite bending moduli - versus two finite, identical bending moduli for the isotropic rod.

\subsection{Discussion}

Our one-dimensional model has been derived under the assumption of slow longitudinal variations of the strain; it is therefore not able to capture microscopic instabilities involving a longitudinal wavelength of order $a$ or less, as studied by Gre37, CK13, CB08, CB86, CDD15. Our bifurcation results are therefore relevant only as long as they appear before a microscopic instability, i.e., for a smaller value of the twisting moment $M_{3}$. By analyzing the microscopic instabilities of twisted ribbons, CB08 have obtained the linear bifurcation condition as

$$
\bar{\tau}_{c}=\frac{4.9}{\sqrt{12}} \sqrt{\bar{T}} \frac{t}{a}+\sqrt{12} \frac{8.6}{\sqrt{1-\nu^{2}}} \quad(\text { microscopic instability, } 1 / q=\mathcal{O}(a))
$$

after converting their notation into ours. In figure 5.2, we compare the threshold for the macroscopic instability from equation (5.1) (thick solid curves), to that for the microscopic instability from equation (5.6) (dashed thin lines). At low values of the tension $T$, the macroscopic instability is seen to occur first. At sufficiently large values of the tension $T$, the macroscopic instability occurs first as well: even though this is not visible from the figure, this follows from comparing the slower growth of the critical twist associated with the macroscopic mode, $\bar{\tau}_{\mathrm{c}} \approx(2 \cdot 181440)^{1 / 6} \bar{T}^{1 / 6}$ for large $\bar{T}$ - as derived by taking the limit $\bar{T} \rightarrow \infty$ in equation (5.1) - to the faster growth of $\bar{\tau}_{c}$ versus $\bar{T}$ for the microscopic mode in equation (5.6). At intermediate values of the tensions, either the microscopic or the macroscopic instability prevails, depending on whether the ratio $a / t$ is large or small, respectively, as can be seen from figure 5.2. 
Anticipating on the results of section 7 , we have also included in figure 5.2 the region where the straight twisted solution is unstable by the formation of a microstructure, which by equation $(7.7)$ occurs for $\left|\bar{\tau}_{\mathrm{c}}\right|=$ $6 \sqrt{2} \sqrt{7 \nu+\sqrt{70-21 \nu^{2}}} \approx 28.1$ for $\nu=0.4$ (shaded area in the figure).

Overall, the figure suggests the following buckling scenario for $\nu=0.4$. For $\sqrt{\bar{T}}$ smaller than $\sim 50$, the macroscopic instability occurs first, thereby validating in retrospect the analysis from section 5.1 . For $\sqrt{\bar{T}}$ larger than $\sim 50$, the figure suggests that a microstructure appears first; however, it is not possible to be conclusive in that case, since the microstructure is a prediction of the one-dimensional ribbon model, which is not designed to resolve wavelengths of order $a$ or less. We hope that a more complete model for ribbons, resolving both short wavelengths and macroscopic instabilities, will be derived in the future.

\section{DERIVATION OF THE EQUiVALENT ROD MODEL}

In this section, we formulate a plate model for the ribbon, valid for finite rotations, and carry out the dimension reduction procedure that delivers the equivalent rod model announced in section 2 .

In our notation, Greek indices are restricted to in-plane directions, as in $\alpha \in\{S, T\}$. Einstein's implicit summation rules for repeated indices are used throughout. We use a comma in subscript to denote partial derivation, as in $f, S(S, T)=\frac{\partial f}{\partial S}(S, T)$.

\subsection{Center-line based representation of the plate}

In section 2.1, the center-line $\boldsymbol{r}(S)$ has been defined as the centroid of the deformed cross-section having Lagrangian coordinate $S$ and the unit tangent $\boldsymbol{d}_{3}(S)$ has been defined as $\boldsymbol{d}_{3}=\boldsymbol{r}^{\prime} /\left|\boldsymbol{r}^{\prime}\right|$, see equation (2.1). Two additional directors $\boldsymbol{d}_{1}(S)$ and $\boldsymbol{d}_{2}(S)$ have been introduced, such that the directors basis $\boldsymbol{d}_{i}(S)$ is orthonormal and direct but we still have to explain precisely how the orientation of $\boldsymbol{d}_{1}(S)$ and $\boldsymbol{d}_{2}(S)$ in the plane perpendicular to $\boldsymbol{d}_{3}(S)$ is fixed.

To build up the plate model representing the thin ribbon, we start by characterizing its deformed configuration. We denote as $\boldsymbol{x}(S, T)$ the final position of the mid-surface point having coordinates $(S, T)$ in reference configuration. We seek $\boldsymbol{x}(S, T)$ in terms of the three displacements functions $w(S, T), u(S, T)$ and $v(S, T)$ in the directors basis,

$$
\boldsymbol{x}(S, T)=\boldsymbol{r}(S)+w(S, T) \boldsymbol{d}_{1}(S)+(T+u(S, T)) \boldsymbol{d}_{2}(S)+v(S, T) \boldsymbol{d}_{3}(S) .
$$

The center-line point $\boldsymbol{r}(S)$ has been defined as the centroid of the cross-section with coordinate $S$ : this implies that the average of any of the displacement functions over $T$ for fixed $S$ is zero,

$$
\forall S \quad\left\{\begin{array}{l}
\int_{-a / 2}^{+a / 2} v(S, T) \mathrm{d} T=0 \\
\int_{-a / 2}^{+a / 2} u(S, T) \mathrm{d} T=0 \\
\int_{-a / 2}^{+a / 2} w(S, T) \mathrm{d} T=0 .
\end{array}\right.
$$

Equation (6.1) alone does not fully specify the unknowns $\boldsymbol{r}, w, u$ and $v$ as a shift in the center-line $\boldsymbol{r}(S)$ function can be compensated by a shift in the displacement functions, without the actual configuration of the ribbon being affected. The role of equation (6.2) is to remove these indeterminacies.

Similarly, the indeterminacy in the choice of the directors $\left(\boldsymbol{d}_{1}, \boldsymbol{d}_{2}\right)$, which are so far free to rotate about the tangent $\boldsymbol{d}_{3}(S)$, is removed by imposing the condition

$$
\forall S \quad \int_{-a / 2}^{+a / 2} T w(S, T) \mathrm{d} T=0 .
$$

This equation warrants that the director $\boldsymbol{d}_{2}(S)$ captures the 'average' twisting angle of the ribbon about the tangent $\boldsymbol{d}_{3}(S)$, where the average is done over the cross-section with coordinate $S$. This parameterization has been used in a number of earlier work on thin elastic rods, see for instance (YH04) and (AL21).

Equations (6.1-6.3) allow the mid-surface of the ribbon to be parameterized in terms of the macroscopic functions $\boldsymbol{r}(S)$ and $\boldsymbol{d}_{i}(S)$, and of the microscopic functions $w(S, T), u(S, T)$ and $v(S, T)$, in a unique way. We call this the center-line based representation of the ribbon.

\subsection{Scaling assumptions}

We need to specify the plate model which we use as a starting point for the dimension reduction. In principle, we could use a general (geometrically exact) plate model, valid for arbitrarily large strain values $\varepsilon$ and $\kappa_{i}$. However using an approximate plate model tailored to a particular range of strain values is much simpler. In the present section, we expand on the dimensional analysis presented in the introduction and identify natural scaling laws for the strain measures $\varepsilon$ and $\kappa_{i}$ of an elastic ribbon.

From the classical theory of elastic rods, we know that, for a rod with rectangular cross-section having dimensions $a \times t$, the stretching modulus is of order $Y a t$, the bending moduli are of order $Y a^{3} t$ (stiff bending mode $\kappa_{1}$ ) and $Y a t^{3}$ (soft bending mode $\kappa_{2}$ ) and the twisting modulus is of order $Y a t^{3}$ (in order of magnitude, the shear modulus is comparable to the Young modulus). We can identify natural orders of magnitude of the strain measures $\varepsilon$ and $\kappa_{i}$ of the ribbon by assuming that all the contributions to the elastic energy are of the same order of magnitude,

$$
Y a t \varepsilon^{2} \sim Y a^{3} t \kappa_{1}^{2} \sim Y a t^{3} \kappa_{2}^{2} \sim Y a t^{3} \kappa_{3}^{2} .
$$


This yields $\varepsilon \sim t \kappa_{2}, \kappa_{1} \sim \frac{t}{a} \kappa_{2}$ and $\kappa_{3} \sim \kappa_{2}$, which sets the relative orders of magnitude of the various strain components. To identify their absolute orders of magnitude, we note that the typical angle of bending of the ribbon, measured over an in-plane distance comparable to the ribbon width $a$, is $\theta \sim a \kappa_{2}$. As is known from, e.g., the Föpplvon Kàrmàn theory of plates, this small rotation is associated with an in-plane strain of order $\varepsilon \sim \theta^{2}$. This yields the natural scale of $\kappa_{2} \sim \frac{t}{a^{2}}$, which is precisely the scale $\kappa^{\star}$ introduced in equation (2.7). To sum up, we have identified the natural scales of the macroscopic strain in a rectangular beam having a slender cross-section $(a \gg t)$ as follows,

$$
\begin{aligned}
\varepsilon & =\mathcal{O}\left(\left(\frac{t}{a}\right)^{2}\right) & & \text { (stretching mode) } \\
\kappa_{1} & =\mathcal{O}\left(\kappa^{\star} \frac{t}{a}\right) & & \text { (stiff bending mode) } \\
\kappa_{2} & =\mathcal{O}\left(\kappa^{\star}\right) & & \text { (soft bending mode) } \\
\kappa_{3} & =\mathcal{O}\left(\kappa^{\star}\right) & & \text { (twisting mode) }
\end{aligned}
$$

These natural scaling assumptions will enable us to use simplified (weakly non-linear) expressions for the strain in the plate model.

These scaling laws are consistent with those discussed in the introduction ( $\$ 1)$, as the dimensionless strain measure introduced in equation (1.1) is nothing but $\bar{\kappa} \sim \kappa \frac{a^{2}}{t} \sim \kappa / \kappa^{\star}$.

\subsection{Energy formulation of the plate model}

To construct the plate model, we start by identifying the membrane strain $\boldsymbol{E}(S, T)$ and the bending strain $\boldsymbol{B}(S, T)$ in terms of the current configuration $\boldsymbol{x}(S, T)$ from equation (6.1); they enter into the stretching and bending energies of the plate, respectively.

With a view of calculating the membrane strains that enter into the plate model, we consider the deformation gradient $\boldsymbol{F}(S, T)$, such that $\mathrm{d} \boldsymbol{x}=\boldsymbol{F}(S, T) \cdot\left(\mathrm{d} S \boldsymbol{e}_{S}+\mathrm{d} T \boldsymbol{e}_{T}\right)$. An approximation for the membrane strain $\boldsymbol{E}^{\dagger}=\frac{1}{2}\left(\boldsymbol{F}^{T} \cdot \boldsymbol{F}-\boldsymbol{I}\right)$ is then obtained as $\boldsymbol{E} \approx \boldsymbol{E}^{\dagger}$, where

$$
\begin{gathered}
\boldsymbol{E}(S, T)=\left(\varepsilon(S)+\kappa_{1}(S) T+\frac{\kappa_{3}^{2}(S)}{2} T^{2}-\kappa_{2}(S) w(S, T)-\kappa_{3}(S) T w, S(S, T)\right) \boldsymbol{e}_{S} \otimes \boldsymbol{e}_{S} \ldots \\
+\frac{\kappa_{3}(S)}{2}\left(w(S, T)-T w_{, T}(S, T)\right)\left(\boldsymbol{e}_{S} \otimes \boldsymbol{e}_{T}+\boldsymbol{e}_{T} \otimes \boldsymbol{e}_{S}\right) \ldots \\
+\left(\frac{u_{\alpha, \beta}(S, T)+u_{\beta, \alpha}(S, T)}{2}+\frac{1}{2} w_{, \alpha}(S, T) w_{, \beta}(S, T)\right) \boldsymbol{e}_{\alpha} \otimes \boldsymbol{e}_{\beta} .
\end{gathered}
$$

In the last term in the right-hand side, there are implicit summations over the Greek indices, which by convention are limited to the in-plane directions, $\alpha, \beta \in\{S, T\}$. We also use the generic notation $\left(u_{T}, u_{S}\right)=(u, v)$ for the inplane components of the displacement.

To derive the approximate expressions $\boldsymbol{E}$ for the membrane strain in equation (6.6), we have derived the geometrically exact expressions for the membrane strain $\boldsymbol{E}^{\dagger}$ first, and have combined them with the scaling assumptions from equation (6.5), retaining only the contributions that are dominant in the limit $t \ll a$, see section A.1 from the appendix. We emphasize that these strain measures are geometrically non-linear, and can handle finite rotations of the director frame $\boldsymbol{d}_{i}(S)$; they do assume, however, that cross-sections bend by a small amount, i.e., the relative rotation at the scale $a$ of a cross-section is assumed to remain small.

To obtain the curvature strain, we compute the projection of the second derivatives $\boldsymbol{x}_{, \alpha \beta}(S)$ onto the unit normal $\boldsymbol{d}_{1}(S)$, keeping only the dominant contributions. This yields the bending strain as

$$
\boldsymbol{B}(S, T)=\left(\kappa_{2}(S)-\kappa_{3}^{\prime}(S) T\right) \boldsymbol{e}_{S} \otimes \boldsymbol{e}_{S}-\kappa_{3}(S)\left(\boldsymbol{e}_{S} \otimes \boldsymbol{e}_{T}+\boldsymbol{e}_{T} \otimes \boldsymbol{e}_{S}\right)+w_{, \alpha \beta}(S, T) \boldsymbol{e}_{\alpha} \otimes \boldsymbol{e}_{\beta},
$$

as shown in section A.1 from the appendix.

Next, we introduce the constitutive laws for a linear isotropic elastic material, identical to those used in the LoveKirchhoff theory of plates; they yield the membrane stress $n_{\alpha \beta}$ and the bending stress $m_{a \beta}$ as

$$
\begin{aligned}
n_{\alpha \beta} & =K\left((1-\nu) E_{\alpha \beta}+\nu E_{\gamma \gamma} \delta_{\alpha \beta}\right) \\
m_{\alpha \beta} & =D\left((1-\nu) B_{\alpha \beta}+\nu B_{\gamma \gamma} \delta_{\alpha \beta}\right) .
\end{aligned}
$$

Here, $K$ and $D$ denote the plate's stretching and bending moduli, respectively,

$$
K=\frac{Y t}{1-\nu^{2}}, \quad D=\frac{Y t^{3}}{12\left(1-\nu^{2}\right)} .
$$

The strain energy of this linearly elastic plate $\Phi=\iint \frac{1}{2}\left(n_{\alpha \beta} E_{\alpha \beta}+m_{\alpha \beta} B_{\alpha \beta}\right) \mathrm{d} T \mathrm{~d} S$ is normally written as a twodimensional integral, but can be rewritten in the form of a one-dimensional integral as in equation (2.5) if we carry out a partial integration with respect to $T, \Phi=\int W \mathrm{~d} S$. This introduces the strain energy per unit length:

$$
W=\int_{-a / 2}^{+a / 2} \frac{1}{2}\left(n_{\alpha \beta} E_{\alpha \beta}+m_{\alpha \beta} B_{\alpha \beta}\right) \mathrm{d} T \quad \text { (thin plate model). }
$$

This completes the presentation of the plate model; it is similar to the Donnell-Mushtari-Vlasov shell model (Yam84). 
The goal of the forthcoming dimension reduction procedure is to turn the two-dimensional strain energy density $W$ into a function of just the macroscopic (one-dimensional) strain measures $\varepsilon$ and $\kappa_{i}$, as in equation (2.6).

\subsection{Analysis of homogeneous solutions}

In this section, the homogeneous solutions of the plate model are analyzed. These are the solutions that are invariant in the longitudinal direction, in the sense that neither the macroscopic strain $\boldsymbol{h}$,

$$
\boldsymbol{h}=\left(\varepsilon, \kappa_{1}, \kappa_{2}, \kappa_{3}\right),
$$

nor the displacement functions depend of $S$. Accordingly, we consider in this section a constant macroscopic strain $\boldsymbol{h}$ as well as displacement solutions $w, u$ and $v$ that are functions of $T$ and $\boldsymbol{h}$ but not of $S$ : we use the notation

$$
w(S, T)=w_{\boldsymbol{h}}(T) \quad u(S, T)=u_{\boldsymbol{h}}(T) \quad v(S, T)=v_{\boldsymbol{h}}(T) .
$$

The subscript $\boldsymbol{h}$ used here serves two purposes: it identifies the solution as being a homogeneous solution, and it makes explicit the dependence of this solution on the macroscopic strain $\boldsymbol{h}=\left(\varepsilon, \kappa_{1}, \kappa_{2}, \kappa_{3}\right)$. With constant macroscopic strain $\boldsymbol{h}$, the center-line is generally a helix but degenerate cases are possible, such as circular or straight center-lines.

When the particular form (6.12) of the displacement is inserted into the expressions (6.6-6.7) of the strain, we find the membrane strain $\boldsymbol{E}=E_{S S}^{\boldsymbol{h}}(T) \boldsymbol{e}_{S} \otimes \boldsymbol{e}_{S}+E_{T T}^{\boldsymbol{h}}(T) \boldsymbol{e}_{T} \otimes \boldsymbol{e}_{T}+E_{S T}^{\boldsymbol{h}}(T)\left(\boldsymbol{e}_{S} \otimes \boldsymbol{e}_{T}+\boldsymbol{e}_{T} \otimes \boldsymbol{e}_{S}\right)$ and the bending strain $\boldsymbol{B}=B_{S S}^{\boldsymbol{h}}(T) \boldsymbol{e}_{S} \otimes \boldsymbol{e}_{S}+B_{T T}^{\boldsymbol{h}}(T) \boldsymbol{e}_{T} \otimes \boldsymbol{e}_{T}+B_{S T}^{\boldsymbol{h}}(T)\left(\boldsymbol{e}_{S} \otimes \boldsymbol{e}_{T}+\boldsymbol{e}_{T} \otimes \boldsymbol{e}_{S}\right)$ for homogeneous solutions as

$$
\begin{array}{ll}
E_{S S}^{\boldsymbol{h}}(T)=\varepsilon+\kappa_{1} T+\frac{\kappa_{3}^{2}}{2} T^{2}-\kappa_{2} w_{\boldsymbol{h}}(T) & B_{S S}^{\boldsymbol{h}}(T)=\kappa_{2} \\
E_{T T}^{\boldsymbol{h}}(T)=u_{\boldsymbol{h}}^{\prime}(T)+\frac{1}{2} w_{\boldsymbol{h}}^{\prime 2}(T) & B_{T T}^{\boldsymbol{h}}(T)=w_{\boldsymbol{h}}^{\prime \prime}(T) \\
E_{S T}^{\boldsymbol{h}}(T)=\frac{\kappa_{3}}{2}\left(w_{\boldsymbol{h}}(T)-T w_{\boldsymbol{h}}^{\prime}(T)\right)+\frac{1}{2} v_{\boldsymbol{h}}^{\prime}(T) & B_{S T}^{\boldsymbol{h}}(T)=-\kappa_{3} .
\end{array}
$$

Here, we use primes for the derivative along the transverse direction $T$ : there is no dependence on $S$ in the context of homogeneous solutions, hence no ambiguity.

Inserting these expressions into the strain energy density (6.10), one obtains a functional

$$
W\left(\boldsymbol{h}, w_{\boldsymbol{h}}(\cdot), v_{\boldsymbol{h}}(\cdot), u_{\boldsymbol{h}}(\cdot)\right)
$$

whose detailed expression is worked out in section A.2 from the appendix. For any prescribed value of the macroscopic strain $\boldsymbol{h}$, we identify the optimum microscopic displacements $w_{\boldsymbol{h}}, v_{\boldsymbol{h}}$ and $u_{\boldsymbol{h}}$ by the condition that they render this functional $W$ stationary, subject to the kinematic constraints (6.2-6.3) ensuring the consistency of the solution with the prescribed macroscopic shape. The constraints are treated using Lagrange multipliers.

This leads to a set of coupled non-linear ordinary differential equations for the microscopic displacements $w_{\boldsymbol{h}}(T)$, $v_{\boldsymbol{h}}(T)$ and $u_{\boldsymbol{h}}(T)$, with coefficients depending on $\boldsymbol{h}$. The detailed solution is worked out in section A.2 in the appendix. This yields a solution for all the components of the displacement $\left(w_{\boldsymbol{h}}(\cdot), v_{\boldsymbol{h}}(\cdot), u_{\boldsymbol{h}}(\cdot)\right)$. In particular the solution for the deflection $w_{\boldsymbol{h}}(T)$ is as announced earlier in equation (2.13). This completes the determination of the homogeneous solution $\left(w_{\boldsymbol{h}}(\cdot), v_{\boldsymbol{h}}(\cdot), u_{\boldsymbol{h}}(\cdot)\right)$.

When the solution for $\left(w_{\boldsymbol{h}}(\cdot), v_{\boldsymbol{h}}(\cdot), u_{\boldsymbol{h}}(\cdot)\right)$ is inserted into the strain energy density (6.14), one obtains the energy of the homogeneous solution as

$$
\begin{aligned}
W\left(\varepsilon, \kappa_{1}, \kappa_{2}, \kappa_{3}\right)=\frac{1}{2} Y a t\left(\varepsilon+\frac{a^{2} \kappa_{3}^{2}}{24}\right)^{2}+\frac{1}{2} & \frac{Y a^{3} t}{12} \kappa_{1}^{2} \ldots \\
& +\frac{1}{2} \frac{Y a t^{3}}{12} \kappa_{2}^{2}+\frac{1}{2} \frac{Y a t^{3}}{6(1+\nu)} \kappa_{3}^{2}+\frac{1}{2} \frac{Y a^{5} t}{2}\left(\nu \kappa_{2}^{2}+\kappa_{3}^{2}\right)^{2} \varphi\left(\frac{\kappa_{2}}{\kappa^{\star}}\right) .
\end{aligned}
$$

We have made good process towards the derivation of our one-dimensional model: the strain energy density above is a function of the one-dimensional strain $\boldsymbol{h}$. Note the key role played by homogeneous solutions in the dimension reduction procedure.

\subsection{Relaxation of the stiff modes}

The above expression for the strain energy $W\left(\varepsilon, \kappa_{1}, \kappa_{2}, \kappa_{3}\right)$ can be used to set up a one-dimensional rod model but it is beneficial to first simplify it further using dimensional analysis, as we do now.

In the limit of a thin ribbon, $t / a \rightarrow 0$, our scaling assumptions in equation (6.5) show that $|\varepsilon| \sim(t / a)^{2} \ll 1$ and $\left|\kappa_{1}\right| \ll \kappa^{\star}$ while $\kappa_{2} \sim \kappa_{3} \sim \kappa^{\star}$ : the axial strain remains very small (in an absolute sense, since this is a dimensionless quantity), and so is the curvature $\kappa_{1}$ of the stiff bending mode (when compared to the natural scale $\kappa^{\star}$, since this quantity has the dimension of the inverse of a length). The fact that $\varepsilon$ and $\kappa_{1}$ remain small means that the corresponding moduli, of order $Y a t$ and $Y a^{3} t$ respectively, are 'large'- to statement can be made accurate using further scaling assumptions on the load, but we prefer to follow a less formal presentation here. In this section, we explore the limit where these two modes are taken to be perfectly stiff. 
In this limit, the variables $\varepsilon$ and $\kappa_{1}$ can be eliminated from the energy by a relaxation procedure: the values of $\varepsilon$ and $\kappa_{1}$ that make $W\left(\varepsilon, \kappa_{1}, \kappa_{2}, \kappa_{3}\right)$ minimum for prescribed $\kappa_{2}$ and $\kappa_{3}$ are first obtained as $\tilde{\varepsilon}\left(\kappa_{3}\right)=-\frac{a^{2} \kappa_{3}^{2}}{24}$ and $\tilde{\kappa}_{1}=0$ from equation (6.15), and then inserted back into $W$ (note that this relaxation step is required: it is not correct to simply insert $\varepsilon=0$ and $\kappa_{1}=0$ into the energy $W\left(\varepsilon, \kappa_{1}, \kappa_{2}, \kappa_{3}\right)$, even if we will ultimately set $\varepsilon=0$ and $\left.\kappa_{1}=0\right)$. This yields a strain energy functional $W\left(\tilde{\varepsilon}\left(\kappa_{3}\right), \tilde{\kappa}_{1}, \kappa_{2}, \kappa_{3}\right)$ which is a function of the soft strain $\kappa_{2}$ and $\kappa_{3}$ only: this is precisely to the strain energy functional $W\left(\kappa_{2}, \kappa_{3}\right)$ announced in equation (2.6). The optimal values of the relaxed variables $\varepsilon=\tilde{\varepsilon}=-\frac{a^{2} \kappa_{3}^{2}}{24}$ and $\kappa_{1}=\tilde{\kappa}_{1}=0$ must be enforced as kinematic constraints in the perfectly stiff model.

Yet another simplification can be introduced as follows. Observing that $\tilde{\varepsilon} \sim(t / a)^{2}$ is a very small number, we use the same relaxed energy but set the first kinematic constraint to $\varepsilon=0$ instead of $\varepsilon=\tilde{\varepsilon}$, keeping the second constraint $\kappa_{1}=0$ unchanged. This typically does not change the solutions of the model significantly. This final simplification yields the model announced in Section 2.

This relaxation completes the justification of the kinematic constraints announced earlier in equation (2.4), as well as the expression for the strain energy $W\left(\kappa_{2}, \kappa_{3}\right)$ in equation (2.6).

\section{CONVEXIFICATION OF THE STRAIN ENERGY}

In this section, we show that the equilibrium solutions to the one-dimensional extensible ribbon model may feature discontinuous strain distributions $\kappa_{2}(S)$ and $\kappa_{3}(S)$. The reason is that the strain energy density $W\left(\kappa_{2}, \kappa_{3}\right)$ is non-convex, leading to a phenomenon akin to phase coexistence, which is analyzed here using a Maxwell-type of construction. A convexified strain energy is constructed, which accounts for the formation of a microscopic mixture in some regions of the parameter space $\left(\kappa_{2}, \kappa_{3}\right)$.

\subsection{Analysis of discontinuities}

We consider an equilibrium solution of the one-dimensional model such that at least one of the functions $\kappa_{2}(S)$ and $\kappa_{3}(S)$ has a discontinuity at some point $S^{\dagger}$. We denote by $\kappa_{i}^{ \pm}=\kappa_{i}\left(S_{ \pm}^{\dagger}\right)$ the value of the strain on either the positive side $S_{+}^{\dagger}$ or the negative side $S_{-}^{\dagger}$ of the discontinuity, and by $\llbracket f \rrbracket=f\left(S_{+}^{\dagger}\right)-f\left(S_{-}^{\dagger}\right)$ the jump of a function $f(S)$ across the discontinuity. We require that the center-line position and the directors remain continuous across the discontinuity, $\llbracket \boldsymbol{r} \rrbracket=\mathbf{0}$ and $\llbracket \boldsymbol{d}_{i} \rrbracket=\mathbf{0}$.

With the aim to characterize these discontinuities, we write and then solve the equilibrium conditions at $S^{\dagger}$. First, the balance of moments imposes that the internal moment $\boldsymbol{M}=M_{i} \boldsymbol{d}_{i}=M_{1} \boldsymbol{d}_{1}+\frac{\partial W}{\partial \kappa_{2}} \boldsymbol{d}_{2}+\frac{\partial W}{\partial \kappa_{3}} \boldsymbol{d}_{3}$ from equation (2.17) is continuous across the discontinuity: in particular, this requires

$$
\llbracket \frac{\partial W}{\partial \kappa_{2}}\left(\kappa_{2}, \kappa_{3}\right) \rrbracket=0 \quad \llbracket \frac{\partial W}{\partial \kappa_{3}}\left(\kappa_{2}, \kappa_{3}\right) \rrbracket=0
$$

At a discontinuity, a third equilibrium condition must be enforced, which warrants that the energy is stationary when the coordinate $S^{\dagger}$ of the discontinuity is perturbed: this corresponds to a perturbation where the domain boundary moves with respect to the body. This condition is known as a Weierstrass-Erdmann condition (Sag92, SF93); it implies the equality of the chemical potential in the theory of phase transition, and reads

$$
\llbracket W\left(\kappa_{2}, \kappa_{3}\right)-\kappa_{2} \frac{\partial W}{\partial \kappa_{2}}\left(\kappa_{2}, \kappa_{3}\right)-\kappa_{3} \frac{\partial W}{\partial \kappa_{3}}\left(\kappa_{2}, \kappa_{3}\right) \rrbracket=0
$$

Equations (7.1-7.2) are non-linear equations for the four values of the strain $\kappa_{2}\left(S_{ \pm}^{\dagger}\right)$ and $\kappa_{3}\left(S_{ \pm}^{\dagger}\right)$ on either side of the singularity. We have not been able to solve these equations in full generality, but we have found particular solutions which are derived as follows. Suppose we can find two constants $\tilde{\kappa}_{2}$ and $\tilde{\kappa}_{3}$ such that the corresponding bending moment vanishes,

$$
\frac{\partial W}{\partial \kappa_{2}}\left(\tilde{\kappa}_{2}, \tilde{\kappa}_{3}\right)=0
$$

As we will check later, there are many such pairs $\left(\tilde{\kappa}_{2}, \tilde{\kappa}_{3}\right)$. For any such pair $\left(\tilde{\kappa}_{2}, \tilde{\kappa}_{3}\right)$, we consider a discontinuity where $\kappa_{2}(S)$ jumps sign from $-\tilde{\kappa}_{2}$ to $\tilde{\kappa}_{2}$ while $\kappa_{3}(S)$ is continuous and equal to $\tilde{\kappa}_{3}$,

$$
\kappa_{2}\left(S_{ \pm}^{\dagger}\right)= \pm \tilde{\kappa}_{2} \quad \kappa_{3}\left(S_{ \pm}^{\dagger}\right)=\tilde{\kappa}_{3}
$$

All discontinuities of the form (7.4) satisfy the equilibrium equations (7.1-7.2), as we show now. The key remark is that the strain energy density $W\left(\kappa_{2}, \kappa_{3}\right)$ is an even function of both its arguments $\kappa_{2}$ and $\kappa_{3}$, implying that $\frac{\partial W}{\partial \kappa_{2}}$ and $\frac{\partial W}{\partial \kappa_{3}}$ are odd and even functions of $\kappa_{2}$, respectively. Since $\frac{\partial W}{\partial \kappa_{2}}$ is odd, we have $\frac{\partial W}{\partial \kappa_{2}}\left(\kappa_{2}\left(S_{-}^{\dagger}\right), \kappa_{3}\left(S_{-}^{\dagger}\right)\right)=\frac{\partial W}{\partial \kappa_{2}}\left(-\tilde{\kappa}_{2}\right.$, $\left.\tilde{\kappa}_{3}\right)=-\frac{\partial W}{\partial \kappa_{2}}\left(\tilde{\kappa}_{2}, \tilde{\kappa}_{3}\right)=0$ by equation (7.3). From equation $(7.3)$, we also have $\frac{\partial W}{\partial \kappa_{2}}\left(\kappa_{2}\left(S_{+}^{\dagger}\right), \kappa_{3}\left(S_{+}^{\dagger}\right)\right)=\frac{\partial W}{\partial \kappa_{2}}\left(\tilde{\kappa}_{2}, \tilde{\kappa}_{3}\right)=0$. This shows that the first equilibrium equation in (7.1) is verified. The second equilibrium equation in (7.1) follows directly from the fact that $\frac{\partial W}{\partial \kappa_{3}}$ is an even function of $\kappa_{2}$. Similarly, the Weierstrass-Erdmann condition (7.2) follows from the fact that $W, \kappa_{2} \frac{\partial W}{\partial \kappa_{2}}$ and $\kappa_{3} \frac{\partial W}{\partial \kappa_{3}}$ are all even functions of $\kappa_{2}$. This completes the proof: singularity of the form (7.4) indeed satisfy all the equilibrium equations. 


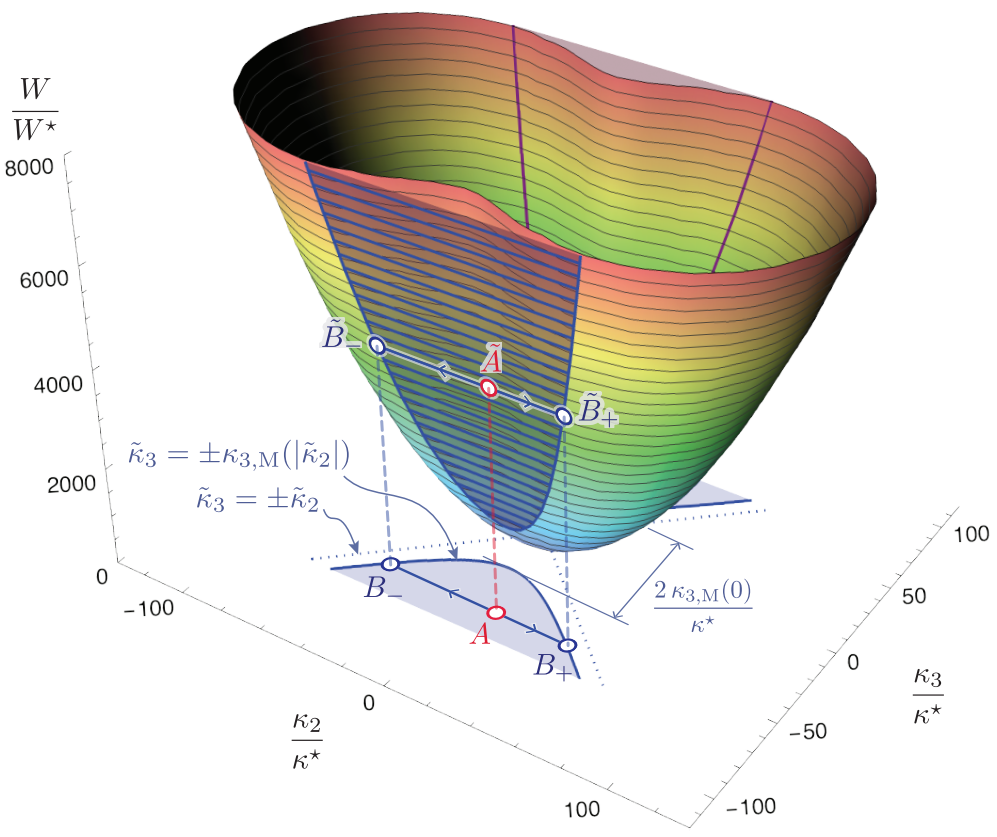

Figure 7.1. Convexified strain energy for $\nu=0.4$. The strain values that coexist across a discontinuity (Maxwell pairs) are denoted as $\left(B_{-}, B_{+}\right)$; they are given by equation (7.3) or equations (7.5-7.6). When lifted onto the energy surface, they yield the points $\left(\tilde{B}_{-}, \tilde{B}_{+}\right)$that sit on the edge of the concave part of the energy surface: replacing this concave part by the surface swept by the segments joining $\tilde{B}_{-}$and $\tilde{B}_{+}$(semi-transparent blue surface in the figure), one obtains the convexified energy surface. In the plane shown at the bottom, the blue shaded regions containing the point $A$ corresponds to macroscopic strain values that give rise to a microscopic mixture of the phases $B_{-}$and $B_{+}$.

In figure 7.1, we have solved equation (7.3) in the $\left(\kappa_{2} / \kappa^{\star}, \kappa_{3} / \kappa^{\star}\right)$ plane: the result is the solid blue curve drawn at the bottom of the figure. A generic point $B_{ \pm}=\left(\tilde{\kappa}_{2} / \kappa^{\star}, \tilde{\kappa}_{3} / \kappa^{\star}\right)$ on this curve can be lifted onto the energy surface to yield a point $\tilde{B}_{ \pm}=\left(\tilde{\kappa}_{2} / \kappa^{\star}, \tilde{\kappa}_{3} / \kappa^{\star}, W\left(\tilde{\kappa}_{2}, \tilde{\kappa}_{3}\right) / W^{\star}\right)$; at any such point, the tangent plane to the energy surface contains a line parallel to the $\kappa_{2}$-axis by equation (7.3). This parallel line connects two points $\tilde{B}_{-}$and $\tilde{B}_{+}$on the energy surface, having opposite values of the bending strain $\kappa_{2}$ and identical values of the twisting strain $\kappa_{3}$ : in view of equation (7.4), the pairs $\left(B_{-}, B_{+}\right)$correspond to values of the strain that can coexist across a discontinuity. We refer to them as Maxwell pairs.

By inserting the explicit form of the energy $W\left(\kappa_{2}, \kappa_{3}\right)$ from equation (2.6) into equation (7.3) and solving with respect to $\tilde{\kappa}_{3}$, we obtain the explicit expression of Maxwell pairs $\left(\tilde{\kappa}_{2}, \tilde{\kappa}_{3}\right)$ as

$$
\tilde{\kappa}_{3}= \pm \kappa_{3, \mathrm{M}}\left(\left|\tilde{\kappa}_{2}\right|\right)
$$

where $\kappa_{3, M}$ is the function defined for $\kappa>0$ by

$$
\kappa_{3, \mathrm{M}}(\kappa)=\left(\frac{\nu \kappa(2 p-q \kappa)+2 \sqrt{\kappa\left(\nu^{2} p^{2} \kappa+\left(1-\nu^{2}\right) q \kappa^{\star 2}\right)}}{q}\right)^{1 / 2} \text { where }\left\{\begin{array}{l}
p=\varphi\left(\frac{\kappa}{\kappa^{\star}}\right) \\
q=\frac{-\varphi^{\prime}\left(\frac{\kappa}{\kappa^{\star}}\right)}{\kappa^{\star}}
\end{array}\right.
$$

It can be shown that the function $\kappa_{3, \mathrm{M}}(\kappa)$ is strictly increasing over the domain $\kappa \geqslant 0$, as suggested by the figure. We will therefore denote as $\kappa_{2, \mathrm{M}}$ the reciprocal function $\kappa_{2, \mathrm{M}}=\left(\kappa_{3, \mathrm{M}}\right)^{(-1)}$ : for any $\kappa_{3} \geqslant \kappa_{3, \mathrm{M}}(0), \kappa_{2, \mathrm{M}}\left(\kappa_{3}\right)$ is the unique $\kappa_{2} \geqslant 0$ such that $\kappa_{3}=\kappa_{3, \mathrm{M}}\left(\kappa_{2}\right)$, i.e., such that equation (7.3) is satisfied.

Using the Taylor expansion $\varphi(v)=\frac{1}{360}-\frac{v^{2}}{181440}+\mathcal{O}\left(v^{4}\right)$, one can calculate the minimum value $\kappa_{3, \mathrm{M}}(0)$ of $\left|\tilde{\kappa}_{3}\right|$ such that a Maxwell pair $\left(\tilde{\kappa}_{2}, \tilde{\kappa}_{3}\right)$ exists as

$$
\kappa_{3, \mathrm{M}}(0)=\kappa^{\star} \times 6 \sqrt{2} \sqrt{7 \nu+\sqrt{70-21 \nu^{2}}} .
$$

This corresponds to the gap $2 \kappa_{3, \mathrm{M}}(0) / \kappa^{\star}$ between the shaded blue regions in the figure.

\subsection{Convexification of the energy}

Consider now a pair of bending and twisting strain $\left(\kappa_{2}, \kappa_{3}\right)$ that lie inside the shaded blue region at the bottom of figure 7.1, for example point $A$. By a classical mixture argument, a solution featuring the strain $\left(\kappa_{2}, \kappa_{3}\right)$ is unstable: its strain energy is lowered if the uniform bending and twisting strain $A=\left(\kappa_{2}, \kappa_{3}\right)$ is replaced by a mixture of microscopic domains corresponding to the strain pairs denoted as $B_{-}$and $B_{+}$in the figure. Both $B_{-}$and $B_{+}$have the same twisting strain $\kappa_{3}$ as the initial point $A$, and their bending strains $\kappa_{2}$ are opposite: $B_{-}$and $B_{+}$are Maxwell pairs. 
Mathematically, this can be restated as follows: the homogeneous solution $A=\left(\kappa_{2}, \kappa_{3}\right)$ is unstable if $\left|\kappa_{3}\right| \geqslant$ $\kappa_{3, \mathrm{M}}\left(\left|\kappa_{2}\right|\right)$ and gives rise to a mixture of phases $B_{ \pm}$where

$$
B_{ \pm}=\left( \pm \kappa_{2, \mathrm{M}}\left(\left|\kappa_{3}\right|\right), \kappa_{3}\right)
$$

This phase decomposition is denoted by the blue arrows in the figure.

The fractions of the phases in the mixture are chosen such that the overall (macroscopic) strain matches the target value $A=\left(\kappa_{2}, \kappa_{3}\right)$. To account for the formation of the mixture, the strain energy $W\left(\kappa_{2}, \kappa_{3}\right)$ of the homogeneous phase $\left(\kappa_{2}, \kappa_{3}\right)$ must be replaced with the energy $W\left(\kappa_{2}, \mathrm{M}\left(\left|\kappa_{3}\right|\right), \kappa_{3}\right)$ of the mixture, which is lower. Graphically, this amounts to replace the original energy surface $W\left(\kappa_{2}, \kappa_{3}\right)$ by its convex hull: the concave parts of the energy surface located above the shaded blue domain must be replaced by the ruled surface shown in semi-transparent blue in the figure.

The convexified energy $W_{\text {conv }}\left(\kappa_{2}, \kappa_{3}\right)$ is defined as the energy of the initial surface in the stable (convex) parts, and as the energy of the mixture in the unstable (concave) parts:

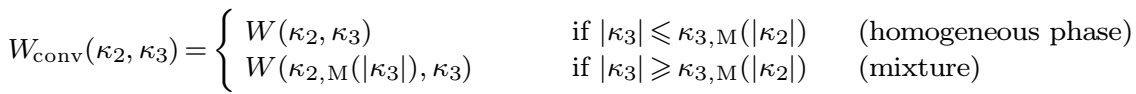

The fact that the construction yields the convex hull of the original energy surface suggests that we have identified in equations (7.3-7.4) all possible of Maxwell points.

\subsection{Consistency with the corrected Sadowsky energy}

We now revisit the above convexification argument in the limit of large strain, $\left|\kappa_{2}\right| \gg \kappa^{\star}$, where we know that the extensible ribbon model is equivalent to Sadowsky inextensible model.

Using equation (2.12), the condition (7.3) defining the Maxwell pairs can be rewritten as $\tilde{\kappa}_{2}-\frac{\tilde{\kappa}_{3}^{4}}{\tilde{\kappa}_{2}^{3}}=0$, which yields $\tilde{\kappa}_{3}= \pm \tilde{\kappa}_{2}$. This yields the following equivalent for the function $\kappa_{3, \mathrm{M}}$ defining the edge of the convex region, see equations $(7.5-7.6)$,

$$
\kappa_{3, \mathrm{M}}\left(\kappa_{2}\right) \simeq \kappa_{2} \text { for } \kappa_{2} \rightarrow+\infty
$$

Obviously, the reciprocal function satisfies $\kappa_{2, \mathrm{M}}\left(\kappa_{3}\right) \simeq \kappa_{3}$ for $\kappa_{3} \rightarrow+\infty$. This corresponds to the dotted, blue asymptotes shown in figure 7.1. We have just recovered the fact that a microstructure is present in Sadowsky model whenever $\left|\kappa_{2}\right|<\left|\kappa_{3}\right|$, as previously shown by FHMP15, PT19.

With the help of equation (2.12), the convexified strain energy in equation (7.8) is rewritten in the large-strain limit as

$$
W_{\text {conv }}\left(\kappa_{2}, \kappa_{3}\right)=\frac{1}{2} \frac{Y a t^{3}}{12\left(1-\nu^{2}\right)} \times\left\{\begin{array}{lll}
\frac{\left(\kappa_{2}^{2}+\kappa_{3}^{2}\right)^{2}}{\kappa_{2}^{2}} & \text { if }\left|\kappa_{3}\right| \leqslant\left|\kappa_{2}\right| & \text { (homogeneous phase) } \\
4 \kappa_{3}^{2} & \text { if }\left|\kappa_{3}\right| \geqslant\left|\kappa_{2}\right| & \text { (mixture) }
\end{array} \quad\left(\text { for }\left|\kappa_{2}\right| \gg \kappa^{\star}\right)\right.
$$

This matches the 'corrected' Sadowsky strain energy functional derived by FHMP15.

\subsection{Discussion}

Here, we have dealt with the non-convex character of the energy $W\left(\kappa_{2}, \kappa_{3}\right)$ by accepting the existence of solutions featuring discontinuous strain. A different approach, which we will explore in future work, is to regularize the model by accounting for the energy cost of the strain gradients $\kappa_{2}^{\prime}(S)$ and $\kappa_{3}^{\prime}(S)$ : in this framework, the discontinuous solutions are replaced by solutions featuring smooth transition regions. Note that the Wunderlich model accounts for this gradient effect (Wun62) but it is limited to the inextensible case.

As noted in equation (7.7), there exists a minimum twisting strain $\kappa_{3}$ where Maxwell pairs can be formed-this is not surprising given that the extensible ribbon model is equivalent at small strain with the linearly elastic rod model, which is convex. Specifically, no discontinuity can exist if the loading is small enough, for $\left|\kappa_{3}\right|<\kappa_{3, \mathrm{M}}(0)$. For $\nu=0.4$, this 'safe' value of the twisting strain is $\kappa_{3, \mathrm{M}}(0)=28.1 \kappa^{\star}$, corresponding to $W=906.3 W^{\star}$. For moderate loading, phase decomposition may therefore not occur at all. We have checked that this is indeed the case for the lateral-torsional buckling analyzed in section 4: the solutions to the buckling problem have been checked to never enter into the non-convex region (blue shaded area in figure 7.1). It was therefore consistent to use the non-convex strain energy for these problems. For the twisted ribbon under tension, figure 5.2 shows that it consistent to use the non-convex strain energy as long as $\sqrt{\bar{T}}$ is less than $\sim 50$, when $\nu=0.4$.

\section{DisCUSSION AND CONCLUSION}

Starting from a non-linear plate theory, we have obtained an equivalent rod model for elastic ribbons. It accounts for finite rotations and features a non-linear constitutive law that captures the stretching and bending of the midsurface, accounting accurately for the deformation of the cross-sections. It is valid for a wide range of thicknessto-width aspect-ratios $t / a$, and is asymptotically equivalent to Kirchhoff's elastic rod model and to Sadowsky's ribbon model in the limits of small and large dimensionless strain $\bar{\kappa}_{i}=\kappa_{i} / \kappa^{\star}$, respectively. We have illustrated this new one-dimensional model by solving two specific buckling problems, and have found that its predictions differ significantly from both the Kirchhoff and Sadowsky models for intermediate values of the dimensionless strain $\bar{\kappa}_{i}$. We have checked that these predictions are in good agreement with both experiments and full shell simulations by the finite-element method.

Even for very thin ribbons, a case where the Sadowsky model is in principle valid, the new one-dimensional model offers key advantages. Mathematically, the Sadowsky model is non-smooth at small strain, see figure 2.2 , and the new model restores a smooth behavior. Physically, the Sadowsky model is inaccurate at small strain (no matter how thin the ribbon is); the new model removes this important limitation thanks to its broader domain of validity. 


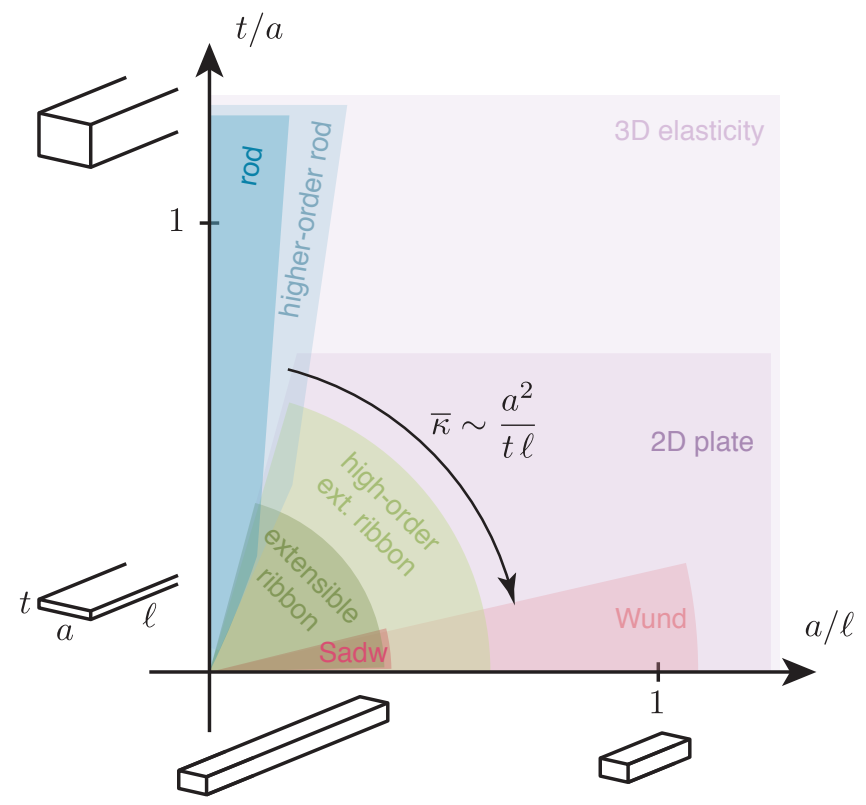

Figure 8.1. Tentative map showing the domains of validity of different theories for a prismatic elastic body having a rectangular cross-section with dimensions $a \times t$ and length $\ell$, and experiencing finite rotations, $\kappa=\mathcal{O}(1 / \ell):$ Kirchhoff theory ('rod'), a typical higher-order rod theory, Sadowsky inextensible ribbon model ('Sadw'), Wunderlich inextensible ribbon model ('Wund'), the present extensible ribbon model, a typical high-order extensible ribbon model (to be derived in future work), a 2D plate model, and the 3D elasticity theory.

In contrast with Vlasov and Wunderlich models (Vla61, Wun62), our one-dimensional model ignores the dependence on the energy on the gradient of twist $\kappa_{3}^{\prime}(S)$. Still, it captures the lateral-torsional buckling accurately (Section 4). The gradient effect would become important for significantly wider ribbons, when $a / \ell$ is not so small. In future work, we will attempt to include it in an extension of the present one-dimensional model.

In figure 8.1, we present a tentative map showing the domains of validity of the different theories for a prismatic elastic body having a rectangular cross-section with dimensions $a \times t$ and length $\ell$. In this diagram, we have assumed finite rotations, $\kappa=\mathcal{O}(1 / \ell)$, implying that the dimensionless bending and twisting strains are of order $\bar{\kappa}=\kappa / \kappa^{\star} \sim 1 /\left(\ell \kappa^{\star}\right) \sim a^{2} /(t \ell)$. Along the vertical axis, for sufficient small values of the width-to-length ratio $a / \ell \ll 1$, one recovers Kirchhoff's classical theory of rods (darker blue region). By using a higher-order rod model (AL21), larger values of $a / \ell$ can be handled with good accuracy (lighter blue region), although the condition $a / \ell \rightarrow 0$ should still hold mathematically. Along the horizontal axis, the Wunderlich model holds for arbitrarily large values of $a / \ell$ (light red domain), and the Sadowsky model holds under the additional assumption $a / \ell \ll 1$ (darker red domain). The one-dimensional model derived here is valid near the origin of the $(a / \ell, t / a)$ plane (darker green domain) and fills the gap between Kirchhoff's rod model and Sadowsky's ribbon model; the dimensionless parameter $\bar{\kappa} \sim a^{2} /(t \ell)$ that governs the transition from Kirchhoff to Sadowsky regime is visually connected to the polar angle in the plane (see figure). The extension of the one-dimensional model accounting for the gradient effect would in principle allow to handle larger values of $a / \ell$ (lighter green domain). The plate model assumes just $t / a \ll 1$ and is valid for any value of $a / \ell$ (darker purple domain): it has a broad range of validity but is significantly more difficult to solve, being a two-dimensional model. As the four ribbon models derive from the plate model, their domain of validity is included in the domain of validity of the plate model. Finally, the three-dimensional theory of elasticity covers all values of the parameters $a / \ell$ and $t / a$ but is even more difficult to solve (lighter purple domain). Note that this map is based on guesswork and will have to be confirmed.

We would like to thank Télémaque Audoly for his help with the experiments, Claire Lestringant for her contribution to the design of the 2D-to-1D reduction method, and Corrado Maurini for sharing his FEniCS-Shells code. Discussions with Vincent Démery, Julien Chopin, and Sébastien Brisard are gratefully acknowledged.

\section{BiBLIOGRAPHY}

[ADK17] V. Agostiniani, A. DeSimone, and K. Koumatos. Shape programming for narrow ribbons of nematic elastomers. Journal of Elasticity, 127:1-24, 2017.

[AL21] B. Audoly and C. Lestringant. Asymptotic derivation of high-order rod models from non-linear 3D elasticity. Journal of the Mechanics and Physics of Solids, 148:104264, 2021.

[AP10] B. Audoly and Y. Pomeau. Elasticity and geometry: from hair curls to the nonlinear response of shells. Oxford University Press, 2010.

[AS15] B. Audoly and K. A. Seffen. Buckling of naturally curved elastic strips: the ribbon model makes a difference. Journal of Elasticity, 119(1):293-320, 2015.

[BFV20] M. Brunetti, A. Favata, and S. Vidoli. Enhanced models for the nonlinear bending of planar rods: localization phenomena and multistability. Proceedings of the Royal Society A: Mathematical, Physical and Engineering Science, 476:20200455, 2020. 
[BNSH14] J. Bae, J.-H. Na, C. D. Santangelo, and R. C. Hayward. Edge-defined metric buckling of temperature-responsive hydrogel ribbons and rings. Polymer, 55:5908-5914, 2014.

[BTQF14] T. Barois, L. Tadrist, C. Quilliet, and Y. Forterre. How a curved elastic strip opens. Physical Review Letters, 113:214301, 2014.

[BWR+08] M. Bergou, M. Wardetzky, S. Robinson, B. Audoly, and E. Grinspun. Discrete elastic rods. ACM Transactions on Graphics (SIGGRAPH), 27(3):63-1, 2008.

[CB86] D. J. Crispino and R. C. Benson. Stability of twisted orthotropic plates. International Journal of Mechanical Sciences, 28(6):371-379, 1986.

[CB08] C. D. Coman and A. P. Bassom. An asymptotic description of the elastic instability of twisted thin elastic plates. Acta Mechanica, 200(1-2):59-68, 2008.

[CBNR20] R. Charrondière, F. Bertails-Descoubes, S. Neukirch, and V. Romero. Numerical modeling of inextensible elastic ribbons with curvature-based elements. Computer Methods in Applied Mechanics and Engineering, 364:112922, 2020.

[CDD15] J. Chopin, V. Démery, and B. Davidovitch. Roadmap to the morphological instabilities of a stretched twisted ribbon. Journal of Elasticity, 119:137-189, 2015.

[CK13] J. Chopin and A. Kudrolli. Helicoids, wrinkles, and loops in twisted ribbons. Physical Review Letters, 111(17):174302, 2013

[Coy90] J. Coyne. Analysis of the formation and elimination of loops in twisted cable. IEEE Journal of Oceanic Engineering, 15(2):72-83, 1990.

[DA14] M. A. Dias and B. Audoly. A non-linear rod model for folded elastic strips. Journal of the Mechanics and Physics of Solids, 62:57-80, 2014

[DA15] M. A. Dias and B. Audoly. "Wunderlich, meet Kirchhoff": a general and unified description of elastic ribbons and thin rods. Journal of Elasticity, 119(1):49-66, 2015

[Dil92] Ellis Harold Dill. Kirchhoff's theory of rods. Archive for History of Exact Sciences, 44(1):1-23, 1992.

[DKK91] E. Doedel, H. B. Keller, and J. P. Kernevez. Numerical Analysis and Control of Bifurcation Problems (I) Bifurcation in Finite Dimensions. International Journal of Bifurcation and Chaos, 1(3):493-520, 1991.

[FHMP15] L. Freddi, P. Hornung, M.-G. Mora, and R. Paroni. A corrected Sadowsky functional for inextensible elastic ribbons. 123:125-136, 2015.

[FHMP18] L. Freddi, P. Hornung, M. G. Mora, and R. Paroni. One-dimensional von Kármán models for elastic ribbons. Meccanica, 53:659-670, 2018.

[FMP04] Lorenzo Freddi, Antonino Morassi, and Roberto Paroni. Thin-walled beams: the case of the rectangular crosssection. Journal of Elasticity, 76(1):45-66, 2004.

[FMP12] L. Freddi, M.-G. Mora, and R. Paroni. Nonlinear thin-walled beams with a rectangular cross-section: part I. Mathematical Models and Methods in Applied Sciences, 22:1150016, 2012.

[GB05] R. Ghafouri and R. Bruinsma. Helicoid to spiral ribbon transition. Physical Review Letters, 94:138101, 2005.

$[$ GMV+17] A. H. Gelebart, D. J. Mulder, M. Varga, A. Konya, G. Vantome, E. W. Meijer, R. L. B. Selinger, and D. J. Broer. Making waves in a photoactive polymer film. Nature, 546:632-636, 2017.

[Gre83] A. G. Greenhill. On the strength of shafting when exposed both to torsion and to end thrust. Proceedings of the Institution of Mechanical Engineers, 34(1):182-225, 1883.

[Gre36] A. E. Green. The equilibrium and elastic stability of a thin twisted strip. Proc. R. Soc. Lond. A, 154:430-455, 1936.

[Gre37] A. E. Green. The elastic stability of a thin twisted strip. II. Proc. R. Soc. Lond. A, 161:197-220, 1937.

[GSD16] D. Grossman, E. Sharon, and H. Diamant. Elasticity and fluctuations of frustrated nanoribbons. Phys. Rev. Lett., 116:258105, 2016.

[HBBM18] J. S. Hale, M. Brunetti, S. P. A. Bordas, and C. Maurini. Simple and extensible plate and shell finite element models through automatic code generation tools. Computers \& Structures, 209:163-181, 2018.

[HF15] D. F. Hinz and E. Fried. Translation of Michael Sadowsky's paper "The differential equations of the Möbius Band". Journal of Elasticity, 119:19-22, 2015.

[Hod06] D. H. Hodges. Nonlinear composite beam theory, volume 213 of Progress in astronautics and aeronautics. American Institute of Aeronautics and Astronautics, 2006.

[LA20] C. Lestringant and B. Audoly. Asymptotically exact strain-gradient models for nonlinear slender elastic structures: a systematic derivation method. Journal of the Mechanics and Physics of Solids, 136:103730, 2020.

[LAK20] C. Lestringant, B. Audoly, and D. M. Kochmann. A discrete, geometrically exact method for simulating nonlinear, elastic or non-elastic beams. Computer Methods in Applied Mechanics and Engineering, 361:112741, 2020.

[Lur05] A. I. Lurie. Theory of Elasticity. Foundations of Engineering Mechanics. Springer, 2005.

[Man73] E. H. Mansfield. Large-deflexion torsion and flexure of initially curved strips. Proceedings of the Royal Society of London. A. Mathematical and Physical Sciences, 334(1598):279-298, 1973.

[MGN18] D. E. Moulton, P. Grandgeorge, and S. Neukirch. Stable elastic knots with no self-contact. Journal of the Mechanics and Physics of Solids, 116:33-53, 2018.

[Mic99] A. G. M. Michell. Elastic stability of long beams under transverse forces. The London, Edinburgh, and Dublin Philosophical Magazine and Journal of Science, 48(292):298-309, 1899.

[Moc01] E. M. Mockensturm. The elastic stability of twisted plates. Journal of Applied Mechanics, 68(4):561-567, 2001.

[Nag63] P. M. Naghdi. A new derivation of the general equations of elastic shells. International Journal of Engineering Science, 1(4):509-522, 1963.

[PBCG16] E. Picault, S. Bourgeois, B. Cochelin, and F. Guinot. A rod model with thin-walled flexible cross-section: extension to 3D motions and application to 3D foldings of tape springs. International Journal of Solids and Structures, 84:64-81, 2016.

[PT19] R. Paroni and G. Tomassetti. Macroscopic and microscopic behavior of narrow elastic ribbons. Journal of Elasticity, 135:409-433, 2019.

[RST+13] M. Ravi Shankar, M. L. Smith, V. P. Tondiglia, K. L. Lee, M. E. McConney, D. W. Wang, L.-S. Tan, and T. J. White. Contactless, photoinitiated snap-through in azobenzene-functionalized polymers. Proceedings of the National Academy of Sciences, 110(47):18792-18797, 2013.

[Sad29] M. Sadowsky. Die Differentialgleichungen des Möbiusschen Bandes. In Jahresbericht der Deutschen Mathematiker-Vereinigung, volume 39 (2. Abt. Heft 5/8), pages 49-51. 1929.

[Sag92] H. Sagan. Introduction to the Calculus of Variations. Dover, 1992

[SF93] D. J. Steigmann and M. G. Faulkner. Variational theory for spatial rods. Journal of Elasticity, 33(1):1-26, 1993.

[Shi92] R. T. Shield. Bending of a beam or wide strip. Quarterly Journal of Mechanics and Applied Mathematics, 45(4):567-573, 1992

[SvdH07] E. L. Starostin and G. H. M. van der Heijden. The shape of a Möbius strip. Nature Materials, 6(8):563-567, 2007 .

[SW19] T. G. Sano and H. Wada. Twist-induced snapping in a bent elastic rod and ribbon. Physical Review Letters, 122:114301, 2019. 
[TBNV19] M. Taffetani, F. Box, A. Neveu, and D. Vella. Limitations of curvature-induced rigidity: how a curved strip buckles under gravity. Europhysics Letters (EPL), 127:14001, 2019.

[TG61] S. Timoshenko and J. M. Gere. Theory of elastic stability. MacGraw Hill, New York, $2^{\text {nd }}$ edition, 1961.

[Vla61] V. Z. Vlasov. Thin-walled elastic beams. Israel Program for Scientific Translations, 1961.

[WRW16] J. J. Wie, M. Ravi Shankar, and T. J. White. Photomotility of polymers. Nature Communications, 7:13260, 2016.

[Wue54] W. Wuest. Einige Anwendungen der Theorie der Zylinderschale. Z. angew. Math. Mech., 34:444-454, 1954.

[Wun62] W. Wunderlich. Über ein abwickelbares möbiusband. Monatshefte für Mathematik, 66(3):276-289, 1962.

[Yam84] N. Yamaki. Elastic stability of circular cylindrical shells, volume 27 of North-Holland series in applied mathematics and mechanics. North Holland, 1984.

[YH04] W. Yu and D. H. Hodges. Elasticity solutions versus asymptotic sectional analysis of homogeneous, isotropic, prismatic beams. Journal of Applied Mechanics, 71:15-23, 2004

[YNI03] Yanlei Yu, Makoto Nakano, and Tomiki Ikeda. Photomechanics: directed bending of a polymer film by light. Nature, 425(6954):145-145, 2003.

\section{Appendix A. Detailed Derivation of the equivalent Rod model}

\section{A.1. Kinematics of the plate model}

By differentiating the center-line based representation of $\boldsymbol{x}(S, T)$ in equation (6.1), one obtains the deformation gradient as

$$
\begin{aligned}
\boldsymbol{F}=\left(1+\varepsilon+v_{, S}-\kappa_{2} w+\right. & \left.\kappa_{1}(T+u)\right) \boldsymbol{d}_{3} \otimes \boldsymbol{e}_{S}+\left(w_{, S}-\kappa_{3}(T+u)+\kappa_{2} v\right) \boldsymbol{d}_{1} \otimes \boldsymbol{e}_{S} \ldots \\
& +\left(u, S+\kappa_{3} w-\kappa_{1} v\right) \boldsymbol{d}_{2} \otimes \boldsymbol{e}_{S}+v, T \boldsymbol{d}_{3} \otimes \boldsymbol{e}_{T}+w, T \boldsymbol{d}_{1} \otimes \boldsymbol{e}_{T}+(1+u, T) \boldsymbol{d}_{2} \otimes \boldsymbol{e}_{T} .
\end{aligned}
$$

Using the geometrically exact definition of the membrane strain $\boldsymbol{E}^{\dagger}=\frac{1}{2}\left(\boldsymbol{F}^{T} \cdot \boldsymbol{F}-\boldsymbol{I}\right)$, we find after some algebra

$$
\begin{aligned}
& E_{S S}^{\dagger}=\frac{1}{2}\left[\left(1+\varepsilon+v, S-\kappa_{2} w+\kappa_{1}(T+u)\right)^{2}+\left(w, S-\kappa_{3}(T+u)+\kappa_{2} v\right)^{2}+\left(u, S+\kappa_{3} w-\kappa_{1} v\right)^{2}-1\right] \\
& E_{T T}^{\dagger}=\frac{1}{2}\left[(1+u, T)^{2}+v_{, T}^{2}+w_{, T}^{2}-1\right] \\
& E_{S T}^{\dagger}=\frac{1}{2}\left[\left(1+\varepsilon+v_{, S}-\kappa_{2} w+\kappa_{1}(T+u)\right) v_{, T}+\left(w_{, S}-\kappa_{3}(T+u)+\kappa_{2} v\right) w_{, T}+\left(u_{, S}+\kappa_{3} w-\kappa_{1} v\right)(1+u, T)\right]
\end{aligned}
$$

The orders of magnitude of the displacement $w, u, v$ are fixed by the orders of magnitude of the strain derived in section 6.2 as follows. The linear contributions to the strain coming from the tangent displacement, such as $v, S$ in $E_{S S}^{\dagger}$ and $u_{, T}$ in $E_{T T}^{\dagger}$, is assumed to be of the same order $\varepsilon \sim(t / a)^{2}$ as the membrane strain from equation (6.5): $v, S \sim u, T \sim(t / a)^{2}$. We also assume that the non-linear contributions to the membrane strain coming from the deflection $w$ are of the same order of magnitude, which yields $w_{, S}^{2} \sim w_{, T}^{2} \sim w, S w, T \sim(t / a)^{2}$. In addition, we assume that all the derivatives in all tangent directions to the mid-surface are of order $1 / a$, i.e.

$$
\frac{\partial}{\partial S} \sim \frac{\partial}{\partial T} \sim \frac{1}{a}
$$

Combining this, we obtain the following estimates for the in-plane displacement and for the deflexion,

$$
(u, v) \sim \frac{t^{2}}{a} \quad w \sim t
$$

They are similar to those underpinning the Föppl-von Kàrmàn equations for plates (AP10).

The scaling relations just obtained allow the membrane strain $\boldsymbol{E}^{\dagger}$ from equation (A.1) to be simplified as follows. Consider for instance the quantity $\tilde{E}_{S S}=\varepsilon+v, S-\kappa_{2} w+\kappa_{1}(T+u)$, appearing in the first square term of $E_{S S}^{\dagger}$. Based on our scaling assumptions, we find $\varepsilon \sim v, S \sim \kappa_{2} w \sim \kappa_{1} T \sim(t / a)^{2}$; the term $\kappa_{1} u$, however, is much smaller in the limit $t / a \rightarrow 0$, as $\kappa_{1} u \sim \frac{t^{2}}{a^{3}} \frac{t^{2}}{a} \sim(t / a)^{4} \ll \varepsilon$. This term is therefore neglected and we use $\tilde{E}_{S S}=\varepsilon+v, S-\kappa_{2} w+\kappa_{1} T+\mathcal{O}\left((t / a)^{4}\right)$. Treating the other terms in $E_{S S}^{\dagger}$ similarly, we find

$$
E_{S S}^{\dagger}=\varepsilon+v_{, S}-\kappa_{2} w+\kappa_{1} T+\frac{1}{2}\left(w, S-\kappa_{3} T\right)^{2}+\mathcal{O}\left(\left(\frac{t}{a}\right)^{4}\right)
$$

which matches the expression proposed in equation (6.6). The expressions for the other strain components can be simplified similarly, and the result is

$$
\begin{aligned}
& E_{S S}(S, T)=\varepsilon+\kappa_{1} T+\frac{\kappa_{3}^{2}}{2} T^{2}-\kappa_{2} w-\kappa_{3} T w, S+\quad v, S \quad+\quad \frac{1}{2} w_{, S}^{2} \\
& E_{T T}(S, T)= \\
& u, T+\frac{1}{2} w_{, T}^{2} \\
& E_{S T}(S, T)=\quad \frac{1}{2} \kappa_{3}(w-T w, T) \\
& +\frac{1}{2}\left(v_{, T}+u, S\right)+\frac{1}{2} w, S w_{, T},
\end{aligned}
$$

as announced earlier in equation (6.6). These expressions are accurate in the sense that $E_{\alpha \beta}^{\dagger}=E_{\alpha \beta}+\mathcal{O}\left((t / a)^{4}\right)$, with $E_{\alpha \beta}^{\dagger} \sim E_{\alpha \beta} \sim(t / a)^{2}$ and $t / a \rightarrow 0$.

To derive the bending strain, we first calculate the tangent vectors $\boldsymbol{x}, S=\boldsymbol{F} \cdot \boldsymbol{e}_{S}$ and $\boldsymbol{x}, T=\boldsymbol{F} \cdot \boldsymbol{e}_{T}$ as

$$
\begin{aligned}
& \boldsymbol{x}, S=\left(1+\varepsilon+v_{, S}-\kappa_{2} w+\kappa_{1}(T+u)\right) \boldsymbol{d}_{3}+\left(w, S-\kappa_{3}(T+u)+\kappa_{2} v\right) \boldsymbol{d}_{1}+\left(u, S+\kappa_{3} w-\kappa_{1} v\right) \boldsymbol{d}_{2} \\
& \boldsymbol{x}_{, T}=v_{, T} \boldsymbol{d}_{3}+w_{, T} \boldsymbol{d}_{1}+(1+u, T) \boldsymbol{d}_{2}
\end{aligned}
$$


as well as their derivatives, which we project on the unit normal $\boldsymbol{d}_{1}$,

$$
\begin{aligned}
& \boldsymbol{x}, S S \cdot \boldsymbol{d}_{1}=\left(1+\varepsilon+v_{, S}-\kappa_{2} w+\kappa_{1}(T+u)\right) \kappa_{2}+\left[w_{, S}-\kappa_{3}(T+u)+\kappa_{2} v\right]_{, S}+\left(u, S+\kappa_{3} w-\kappa_{1} v\right)\left(-\kappa_{3}\right) \\
& \boldsymbol{x}, T T \cdot \boldsymbol{d}_{1}=w_{, T T} \\
& \boldsymbol{x}, S T \cdot \boldsymbol{d}_{1}=\kappa_{2} v_{, T}+w, S T+(1+u, T)\left(-\kappa_{3}\right) .
\end{aligned}
$$

The bending strain measures are obtained by retaining the terms that are of order $w, \alpha \beta \sim \frac{t}{a^{2}}$ : this yields

$$
\begin{aligned}
B_{S S}(S, T)= & \kappa_{2}(S)-\kappa_{3}^{\prime}(S) T+w_{, S S}(S, T) \\
B_{T T}(S, T)= & w_{, T T}(S, T) \\
B_{S T}(S, T)= & -\kappa_{3}(S)+w_{, S T}(S, T)
\end{aligned}
$$

as announced earlier in equation (6.7).

\section{A.2. Homogeneous solution}

In this section, we identify the homogeneous displacement $\left(w_{\boldsymbol{h}}(\cdot), u_{\boldsymbol{h}}(\cdot), v_{\boldsymbol{h}}(\cdot)\right)$ corresponding to a prescribed macroscopic strain $\boldsymbol{h}=\left(\varepsilon, \kappa_{1}, \kappa_{2}, \kappa_{3}\right)$. We do so by calculating the stationary points $(w(\cdot), v(\cdot), u(\cdot))=\left(w_{\boldsymbol{h}}(\cdot), v_{\boldsymbol{h}}(\cdot), u_{\boldsymbol{h}}(\cdot)\right)$ of the strain energy for homogeneous solutions $W(\boldsymbol{h}, w(\cdot), v(\cdot), u(\cdot))$ introduced in equation (6.14). Since the displacement is subjected to the four kinematic constraints in equations (6.2-6.3), we introduce four scalar Lagrange multipliers $\left(f_{S}^{\boldsymbol{h}}, f_{T}^{\boldsymbol{h}}, f_{\mathrm{n}}^{\boldsymbol{h}}, g_{\mathrm{n}}^{\boldsymbol{h}}\right)$, and set to zero the first variation of the augmented energy

$$
-W(\boldsymbol{h}, w(\cdot), v(\cdot), u(\cdot))+\int_{-\frac{a}{2}}^{+\frac{a}{2}}\left[f_{S}^{\boldsymbol{h}} v(T)+f_{T}^{\boldsymbol{h}} u(T)+\left(f_{\mathrm{n}}^{\boldsymbol{h}}+g_{\mathrm{n}}^{\boldsymbol{h}} T\right) w(T)\right] \mathrm{d} T,
$$

with respect to $(w(\cdot), v(\cdot), u(\cdot))$.

This yields

$$
\begin{aligned}
& \forall(\hat{w}(\cdot), \hat{v}(\cdot), \hat{u}(\cdot)) \quad-\int_{-\frac{a}{2}}^{+\frac{a}{2}}\left[n_{S S}(T) \hat{E}_{S S}(T)+n_{T T}(T) \hat{E}_{T T}(T)+2 n_{S T}(T) \hat{E}_{S T}(T)+m_{S S}(T) \hat{B}_{S S}(T) \ldots\right. \\
& \left.+m_{T T}(T) \hat{b}_{T T}(T)+2 m_{S T}(T) \hat{B}_{S T}(T)\right] \mathrm{d} T+\int_{-\frac{a}{2}}^{+\frac{a}{2}}\left[f_{S}^{\boldsymbol{h}} \hat{v}(T)+f_{T}^{\boldsymbol{h}} \hat{u}(T)+\left(f_{\mathrm{n}}^{\boldsymbol{h}}+g_{\mathrm{n}}^{\boldsymbol{h}} T\right) \hat{w}(T)\right] \mathrm{d} T=0
\end{aligned}
$$

where $n_{\alpha \beta}(T)$ and $m_{\alpha \beta}(T)$ are the membrane and bending strain found by inserting the strain associated with the unknowns $(w(\cdot), v(\cdot), u(\cdot))$ into the constitutive law (6.8), and $\hat{E}_{\alpha \beta}$ and $\hat{B}_{\alpha \beta}$ are the virtual changes of strain associated with the perturbation $(\hat{w}, \hat{u}, \hat{v})$. The latter is found by line-arising the strain in equation (6.13) and yields

$$
\begin{aligned}
& \forall(\hat{w}, \hat{v}, \hat{u}) \quad-\int_{-\frac{a}{2}}^{+\frac{a}{2}}\left[f_{S}^{h} \hat{v}(T)-n_{S T}(T) \hat{v}^{\prime}(T)+f_{T}^{\boldsymbol{h}} \hat{u}(T)-n_{T T}(T) \hat{u}^{\prime}(T) \ldots\right. \\
& \left.+\left(\kappa_{2} n_{S S}(T)-\kappa_{3} n_{S T}(T)+f_{\mathrm{n}}^{\boldsymbol{h}}+g_{\mathrm{n}}^{\boldsymbol{h}} T\right) \hat{w}(T)-\left(n_{T T}(T) w^{\prime}(T)-\kappa_{3} T n_{S T}(T)\right) \hat{w}^{\prime}(T)-m_{T T}(T) \hat{w}^{\prime \prime}(T)\right] \mathrm{d} T=0
\end{aligned}
$$

Using two integrations by parts, one obtains the equations of equilibrium as

$$
\begin{aligned}
n_{S T}^{\prime}(T)+f_{S}^{h} & =0 \\
n_{T T}^{\prime}(T)+f_{T}^{h} & =0 \\
q_{T}^{\prime}(T)+\kappa_{2} n_{S S}(T)-\kappa_{3} n_{S T}(T)+f_{\mathrm{n}}^{h}+g_{\mathrm{n}}^{h} T & =0
\end{aligned}
$$

where $q_{T}(T)$ is the shear force,

$$
q_{T}(T)=-\kappa_{3} T n_{S T}(T)-m_{T T}^{\prime}(T)+n_{T T}(T) w^{\prime}(T)
$$

The integration by parts also yields the boundary conditions on the free lateral boundary as

$$
\begin{aligned}
n_{T T}\left(S, \pm \frac{a}{2}\right) & =0 \\
n_{S T}\left(S, \pm \frac{a}{2}\right) & =0 \\
q_{T}\left(S, \pm \frac{a}{2}\right) & =0 \\
m_{T T}\left(S, \pm \frac{a}{2}\right) & =0
\end{aligned}
$$

Integrating the equation of transverse equilibrium $n_{\alpha T}^{\prime}(T)+f_{\alpha}^{\boldsymbol{h}}=0$ and using the boundary conditions $n_{\alpha T}(S$, $\left.\pm \frac{a}{2}\right)=0$, we find two of the four Lagrange multipliers as $f_{T}^{h}=f_{S}^{h}=0$, as well two out of the three components of the membrane strain,

We are left with

$$
\forall T \quad n_{T T}(T)=n_{S T}(T)=0 .
$$

$$
\begin{aligned}
-m_{T T}^{\prime \prime}(T)+\kappa_{2} n_{S S}(T)+f_{\mathrm{n}}^{\boldsymbol{h}}+g_{\mathrm{n}}^{\boldsymbol{h}} T & =0 \\
m_{T T}^{\boldsymbol{h}}( \pm a / 2) & =0 \\
-m_{T T}^{\boldsymbol{h}}( \pm a / 2) & =0
\end{aligned}
$$


Combining the solution (A.11) the constitutive relations for $n_{T T}$ and $n_{S T}(T)$ in equation (6.8) one can obtain the expressions of $v^{\prime}(T)$ and $u^{\prime}(T)$ in terms of $w(T), w^{\prime}(T)$ and the macroscopic strain $\varepsilon$ and $\kappa_{i}$. Combining the remaining constitutive relations and the definition of the strain applicable to the homogeneous case in equations (6.13), and eliminating $v^{\prime}(T)$ and $u^{\prime}(T)$, one obtains

$$
\begin{aligned}
& n_{S S}(T)=Y t\left(\varepsilon+T \kappa_{1}+\frac{T^{2}}{2} \kappa_{3}^{2}-\kappa_{2} w(T)\right) \\
& m_{T T}(T)=Y t a^{4}\left(\kappa^{\star}\right)^{2}\left(\nu \kappa_{2}+w^{\prime \prime}(T)\right)
\end{aligned}
$$

Inserting into equation (A.12), this yields the following boundary-value problem for $w(T)$ :

$$
\begin{gathered}
-Y t a^{4}\left(\kappa^{\star}\right)^{2} w^{\prime \prime \prime \prime}(T)+Y t \kappa_{2}\left(\varepsilon+T \kappa_{1}+\frac{T^{2}}{2} \kappa_{3}^{2}-\kappa_{2} w(T)\right)+f_{\mathrm{n}}^{\boldsymbol{h}}+g_{\mathrm{n}}^{\boldsymbol{h}} T=0 \\
\nu \kappa_{2}+w^{\prime \prime}( \pm a / 2)=0 \\
w^{\prime \prime \prime}( \pm a / 2)=0
\end{gathered}
$$

By integrating the differential equation and using the boundary conditions $w^{\prime \prime \prime}( \pm a / 2)$ and the kinematic constraint $\int_{-a / 2}^{+a / 2} w(S, T) \mathrm{d} T=0$ from equation (6.2), we find

$$
f_{\mathrm{n}}^{h}=-Y t \kappa_{2}\left(\varepsilon+\frac{a^{2}}{24} \kappa_{3}^{2}\right)
$$

Repeating the same argument but multiplying the left-hand side of the differential equation by $T$ first, using the kinematic constraint $\int_{-a / 2}^{+a / 2} T w(S, T) \mathrm{d} T=0$ from equation (6.3), as well as the other boundary condition $w^{\prime \prime}( \pm a / 2)=-\nu \kappa_{2}$, we obtain

$$
g_{\mathrm{n}}^{\boldsymbol{h}}=-Y t \kappa_{2} \kappa_{1} .
$$

In view of this, the boundary value problem for $w(T)$ is rewritten as

$$
\begin{gathered}
a^{4} w^{\prime \prime \prime \prime}(T)+\left(\frac{\kappa_{2}}{\kappa^{\star}}\right)^{2} w(T)=\frac{\kappa_{2} \kappa_{3}^{2}}{2\left(\kappa^{\star}\right)^{2}}\left(T^{2}-\frac{a^{2}}{12}\right) \\
\nu \kappa_{2}+w^{\prime \prime}( \pm a / 2)=0 \\
w^{\prime \prime \prime}( \pm a / 2)=0
\end{gathered}
$$

The solutions of the homogeneous equation $a^{4} w^{\prime \prime \prime \prime}(T)+\left(\frac{\kappa_{2}}{\kappa^{\star}}\right)^{2} w(T)=0$ are a combination of the four functions $\cos \frac{q T}{a} \cosh \frac{q T}{a}, \cos \frac{q T}{a} \sinh \frac{q T}{a}, \sin \frac{q T}{a} \cosh \frac{q T}{a}, \sin \frac{q T}{a} \sinh \frac{q T}{a}$ where $q$ is a scaled wavenumber,

$$
q=\sqrt{\frac{\left|\kappa_{2}\right|}{2 \kappa^{\star}}}
$$

To obtain the solution $w(T)=w_{\boldsymbol{h}}(T)$ of the boundary value problem (A.13), we implemented the method of the variation of the constant in a symbolic calculation language. The constants of integration were set by using the kinematic constraints $\int_{-a / 2}^{+a / 2} w(S, T) \mathrm{d} T=0$ and $\int_{-a / 2}^{+a / 2} T w(S, T) \mathrm{d} T=0$ from equations (6.2-6.3). The result is given in equation (2.13) in the main text.

It is then possible to evaluate the strain energy of this homogeneous solution by inserting it into equation (6.10). After discarding the terms that are zero by equation (A.11), we find

$$
W\left(\varepsilon, \kappa_{1}, \kappa_{2}, \kappa_{3}\right)=\frac{1}{2} \int_{-\frac{a}{2}}^{+\frac{a}{2}}\left[n_{S S}(T) E_{S S}(T)+m_{\alpha \beta}(T) B_{\alpha \beta}(T)\right] \mathrm{d} T,
$$

where $n_{S S}(T)=K\left(E_{S S}+\nu E_{T T}\right)=K\left(1-\nu^{2}\right) E_{S S}=Y t E_{S S}$ since $0=n_{T T}(T)=K\left(\nu E_{S S}+E_{T T}\right)$. Inserting the expression of $w_{\boldsymbol{h}}(T)$ just found in the integral above, and carrying out the integration with the help of a symbolic calculation language, one obtains the expression of $W$ announced in equation (6.15).

\section{Appendix B. Numerical approximation of $\varphi$}

In the numerical illustration presented in Section 4, we solve the boundary-value problem described in section 2.5 using the numerical continuation library AUTO-07p, using Lagrange polynomials and collocation points (DKK91). Other strategies are possible, such as adapting the Discrete elastic rods method (BWR $+08, \mathrm{LAK} 20$ ), or using using curvature-based discrete elements (CBNR20), but this is left for future work.

To avoid the apparent singularity near $v=0$ in the definition (2.8) of $\varphi$ while maintaining good accuracy, we use the following piecewise approximation to the function $\varphi$ in the numerical simulations,

$$
\varphi \text { approx. }(v)=\left\{\begin{array}{ll}
\frac{1}{360}-\frac{v^{2}}{181440}+\frac{2879 v^{4}}{261534873600} & \text { if }|v| \leqslant 0.3 \\
\varphi(v) & \text { if } 0.3 \leqslant|v| \leqslant 1800 \\
2 \sqrt{2}\left(-2+\sqrt{\frac{|v|}{2}}\right)|v|^{-5 / 2} & \text { if } 1800 \leqslant|v|
\end{array} .\right.
$$


The original expression $\varphi(v)$ from equation $(2.8)$ is used for intermediate values $0.3 \leqslant|v| \leqslant 1800$. The orders of the expansions and the transition values 0.3 and 1800 have been chosen in such a way that a uniform relative error $\left|\varphi(v) / \varphi_{\operatorname{approx}}(v)-1\right|<1.1 \times 10^{-11}$ is achieved. The above expression has been used to generate the numerical results shown in the paper.

Alternatively, one may approximate $\varphi$ using its Padé approximant,

$$
\varphi_{\text {Padé }}(v)=\frac{u_{0}+u_{2} v^{2}+u_{4} v^{4}}{r_{0}+r_{2} v^{2}+r_{4} v^{4}+r^{6} v^{6}}
$$

with $u_{0}=10967300738634240, u_{2}=931812257376, u_{4}=3644618$, and $r_{0}=3948228265908326400, r_{2}=81692386545$ $36960, r_{4}=563633048160, r_{6}=1822309$. The benefit is that it avoids introducing piecewise approximations, which might be beneficial for some differential equations integrators, but the maximum relative error is relatively large, of order $5 \%$.

\section{Appendix C. General linearization of a beam problem}

We proceed to linearize the equilibrium boundary value problem of section 2.5 about a known base solution. The procedure is standard, and is adapted from previous work: the reader is referred for instance to the work of DA14 for a detailed justification of the equations presented in this section.

The linear bifurcation analysis is set up by introducing a known base solution, denoted by $(\cdot)^{\dagger}$, and a perturbed solution, denoted by $\widetilde{(\cdot)}$ :

$$
\begin{aligned}
\boldsymbol{r}(S) & =\boldsymbol{r}^{\dagger}(S)+\tilde{\boldsymbol{r}}(S) \\
\boldsymbol{d}_{i}(S) & =\boldsymbol{d}_{i}^{\dagger}(S)+\tilde{\boldsymbol{\psi}}(S) \times \boldsymbol{d}_{i}^{\dagger}(S) \\
\boldsymbol{\kappa}(S) & =\boldsymbol{\kappa}^{\dagger}(S)+\tilde{\boldsymbol{\kappa}}(S) \\
\kappa_{i}(S) & =\kappa_{i}^{\dagger}(S)+\tilde{\kappa_{i}}(S) \\
\boldsymbol{M}(S) & =\boldsymbol{M}^{\dagger}(S)+\tilde{\boldsymbol{M}}(S) \\
M_{i}(S) & =M_{i}^{\dagger}(S)+\tilde{M}_{i}(S) \\
\boldsymbol{R}(S) & =\boldsymbol{R}^{\dagger}(S)+\tilde{\boldsymbol{R}}(S) \\
\boldsymbol{f}(S) & =\boldsymbol{f}^{\dagger}(S)+\tilde{\boldsymbol{f}}(S)
\end{aligned}
$$

Note that $\tilde{\kappa_{i}}(S)$ denotes the perturbations to the strain measures $\kappa_{i}=\boldsymbol{\kappa} \cdot \boldsymbol{d}_{i}$ and not the projection of the perturbed rotation gradient $\tilde{\boldsymbol{\kappa}}$ onto the director $\boldsymbol{d}_{i}$. Likewise, $\widetilde{M}_{i}$ denotes the perturbation to the bending and twisting moments $M_{i}=\boldsymbol{M} \cdot \boldsymbol{d}_{i}$ and not the projection of $\tilde{\boldsymbol{M}}$ onto $\boldsymbol{d}_{i}$. Besides, the perturbed directors are parameterized using an infinitesimal rotation $\tilde{\psi}(S)$, warranting the orthonormality of the perturbed directors frame.

In addition, the infinitesimal rotation is decomposed onto the original basis of directors as

$$
\tilde{\boldsymbol{\psi}}(S)=\tilde{\psi}_{i}(S) \boldsymbol{d}_{i}^{\dagger}(S) \text {. }
$$

The products of two or more quantities bearing a bar are systematically neglected in this linear analysis. The geometric definition of the bending and twisting strain $\kappa_{i}(S)$ in equation (2.3) yields, upon linearization,

$$
\tilde{\kappa_{i}}(S)=\frac{\mathrm{d} \tilde{\psi}_{i}}{\mathrm{~d} S}+\boldsymbol{\kappa}^{\dagger}(S) \cdot\left(\tilde{\boldsymbol{\psi}}(S) \times \boldsymbol{d}_{i}^{\dagger}(S)\right) .
$$

In incremental form, the non-bendability constraint $\kappa_{1}(S)=0$ reads

$$
\tilde{\kappa_{1}}(S)=0 \text {. }
$$

The compatibility condition $\boldsymbol{r}^{\prime}(S)=\boldsymbol{d}_{3}(S)$ is linearized as

$$
\tilde{\boldsymbol{r}}^{\prime}(S)=\tilde{\boldsymbol{\psi}}(S) \times \boldsymbol{d}_{3}^{\dagger}(S),
$$

and the equilibrium equations (2.16) are linearized as

$$
\begin{aligned}
\tilde{\boldsymbol{R}}^{\prime}(S)+\tilde{\boldsymbol{f}}(S) & =\mathbf{0} \\
\tilde{\boldsymbol{M}}^{\prime}(S)+\left(\tilde{\boldsymbol{\psi}}(S) \times \boldsymbol{d}_{3}^{\dagger}(S)\right) \times \boldsymbol{R}^{\dagger}(S)+\boldsymbol{d}_{3}^{\dagger}(S) \times \tilde{\boldsymbol{R}}(S) & =\mathbf{0} .
\end{aligned}
$$

In addition, the perturbation to the internal moment $\tilde{\boldsymbol{M}}(S)$ and the perturbations to the bending and twisting moments $\tilde{M}_{i}(S)$ are connected by

$$
\tilde{\boldsymbol{M}}(S)=\tilde{M}_{i}(S) \boldsymbol{d}_{i}^{\dagger}(S)+\tilde{\boldsymbol{\psi}}(S) \times \boldsymbol{M}^{\dagger}(S) .
$$

These equations must be complemented by the relevant boundary conditions, and by the incremental constitutive law which links the perturbations $\widetilde{M}_{2}(S)$ and $\widetilde{M}_{3}(S)$ to the perturbations $\tilde{\kappa_{2}}(S)$ and $\tilde{\kappa_{3}}(S)$.

\section{ApPendix D. Linearized ANALYsis of lateral torsional BUCKLing}

\section{D.1. Fundamental solution}

The base, unbuckled, solution of the boundary-value problem stated in section 4.1 is $\boldsymbol{r}^{\dagger}(S)=S \boldsymbol{e}_{z}, \boldsymbol{d}_{1}^{\dagger}(S)=\boldsymbol{e}_{x}$, $\boldsymbol{d}_{2}^{\dagger}(S)=\boldsymbol{e}_{y}, \boldsymbol{d}_{3}^{\dagger}(S)=\boldsymbol{e}_{z}$. Accordingly, the rotation gradient is zero, $\boldsymbol{\kappa}^{\dagger}(S)=\mathbf{0}$, and so are the bending and twisting strains, $\kappa_{i}^{\dagger}(S)=0$. The internal moment and internal force are found by solving the equilibrium as $\boldsymbol{M}^{\dagger}(S)=M_{1}^{\dagger}(S) \boldsymbol{e}_{x}$ where $M_{1}^{\dagger}(S)=\frac{1}{2} \varrho g(\ell-S)^{2}$ and $\boldsymbol{R}^{\dagger}(S)=R_{2}^{\dagger}(S) \boldsymbol{e}_{y}$ where $R_{2}^{\dagger}(S)=-\varrho g(\ell-S)$. 


\section{D.2. Linearized equilibrium}

In our particular geometry, $\boldsymbol{\kappa}^{\dagger}(S)=\mathbf{0}$ and equation (C.1) becomes

$$
\tilde{\kappa_{i}}(S)=\frac{\mathrm{d} \tilde{\psi}_{i}}{\mathrm{~d} S} .
$$

In particular, the linearized constitutive constraint $\tilde{\kappa_{1}}(S)=0$ yields $\frac{\mathrm{d} \widetilde{\psi_{1}}}{\mathrm{~d} S}(S)=0$ which, together with the clamping condition $\widetilde{\psi_{1}}(0)=0$ yields

$$
\widetilde{\psi_{1}}(S)=0 \quad \text { for all } S
$$

The applied load does not vary, $\tilde{\boldsymbol{f}}(S)=\mathbf{0}$, implying $\tilde{\boldsymbol{R}}^{\prime}(S)=\mathbf{0}$ by equation (C.4). The stress-free boundary condition writes, in incremental form $\tilde{\boldsymbol{R}}(\ell)=0$. Together with the differential equation $\tilde{\boldsymbol{R}}^{\prime}(S)=\mathbf{0}$, this shows that the internal force does not vary, $\tilde{\boldsymbol{R}}(S)=\mathbf{0}$ for all $S$.

In view of the expressions of the base force and moment, equation (C.5) yields

$$
\tilde{\boldsymbol{M}}(S)=\widetilde{M}_{1}(S) \boldsymbol{e}_{x}+\left(\widetilde{M}_{2}(S)+M_{1}^{\dagger}(S) \widetilde{\psi}_{3}(S)\right) \boldsymbol{e}_{y}+\left(\widetilde{M_{3}}(S)-M_{1}^{\dagger}(S) \widetilde{\psi}_{2}(S)\right) \boldsymbol{e}_{z} .
$$

Inserting into the balance of moments, equation (C.4), which can be simplified to $\tilde{\boldsymbol{M}}^{\prime}(S)+\left(\tilde{\boldsymbol{\psi}}(S) \times \boldsymbol{d}_{3}^{\dagger}(S)\right) \times \boldsymbol{R}^{\dagger}(S)=$ $\mathbf{0}$, we find

$$
\begin{aligned}
\frac{\mathrm{d} \widetilde{M}_{1}}{\mathrm{~d} S} & =0 \\
\frac{\mathrm{d}}{\mathrm{d} S}\left(\widetilde{M}_{2}(S)+M_{1}^{\dagger}(S) \widetilde{\psi}_{3}(S)\right) & =0 \\
\frac{\mathrm{d}}{\mathrm{d} S}\left(\widetilde{M}_{3}(S)-M_{1}^{\dagger}(S) \widetilde{\psi}_{2}(S)\right)+R_{2}^{\dagger}(S) \widetilde{\psi}_{2}(S) & =0 .
\end{aligned}
$$

Together with the incremental stress-free boundary condition $0=\tilde{\boldsymbol{M}}(\ell) \cdot \boldsymbol{e}_{y}=\widetilde{M}_{2}(\ell)+M_{1}^{\dagger}(\ell) \widetilde{\psi}_{3}(\ell)$, the second equation yields

$$
\widetilde{M}_{2}(S)=-M_{1}^{\dagger}(S) \widetilde{\psi_{3}}(S)
$$

Using the balance of moments for the base solution, $\left(M_{1}^{\dagger}\right)^{\prime}-R_{2}^{\dagger}=0$, we simplify the third equation above as

$$
\frac{\mathrm{d} \widetilde{M}_{3}}{\mathrm{~d} S}-M_{1}^{\dagger}(S) \frac{\mathrm{d} \widetilde{\psi}_{2}}{\mathrm{~d} S}=0 \quad \widetilde{M}_{3}(\ell)=0 .
$$

Here, we have included the incremental stress-free boundary condition $0=\tilde{\boldsymbol{M}}(\ell) \cdot \boldsymbol{e}_{z}=\widetilde{M}_{3}(\ell)-M_{\chi}^{\dagger}(\ell) \widetilde{\psi}_{2}(\ell)=\widetilde{M}_{3}(\ell)$. Finally, the clamping condition imposes

$$
\widetilde{\psi}_{3}(0)=0 .
$$

\section{D.3. Cases of the extensible ribbon model and of the linearly elastic model}

We proceed to combine the linearized equilibrium (D.3) and the kinematic equation (D.4) with the linearized constitutive law. We start by addressing the general extensible ribbon model from section 2 . Its linearized constitutive law about the unbuckled configuration $\boldsymbol{\kappa}^{\dagger}(S)=\mathbf{0}$ writes, from equation $(2.17)$

$$
\begin{aligned}
& \widetilde{M_{2}}(S)=Y I_{2} \tilde{\kappa_{2}}(S) \\
& \widetilde{M}_{3}(S)=\mu J \tilde{\kappa_{3}}(S)
\end{aligned}
$$

This is identical to the constitutive law of the linearly elastic model: the present linearized bifurcation analysis applies to both the general extensible ribbon model and to the linearly elastic model.

Inserting the constitutive law (D.5) into the equilibrium equation (D.3), using the expression of the linearized strain in equation (D.1) and the expression of $\widetilde{M}_{2}$ from equation (D.2), we obtain

$$
\begin{gathered}
\mu J \frac{\mathrm{d}^{2} \widetilde{\psi_{3}}}{\mathrm{~d} S^{2}}-M_{1}^{\dagger}(S) \frac{\mathrm{d} \widetilde{\psi_{2}}(S)}{\mathrm{d} S}=0 \quad \frac{\mathrm{d} \widetilde{\psi_{3}}}{\mathrm{~d} S}(\ell)=0 \\
\frac{\mathrm{d} \widetilde{\psi_{2}}}{\mathrm{~d} S}=-\frac{M_{1}^{\dagger}(S)}{Y I_{2}} \widetilde{\psi}_{3}(S)
\end{gathered}
$$

Eliminating $\widetilde{\psi_{2}}$, and recalling the boundary condition (D.4), we obtain the linear boundary-value problem

$$
\frac{\mathrm{d}^{2} \widetilde{\psi}_{3}}{\mathrm{~d} S^{2}}+\frac{\left[M_{1}^{\dagger}(S)\right]^{2}}{Y I_{2} \cdot \mu J} \widetilde{\psi}_{3}(S)=0 \quad \widetilde{\psi}_{3}(0)=0 \quad \frac{\mathrm{d} \widetilde{\psi}_{3}}{\mathrm{~d} S}(\ell)=0
$$

which is recast into dimensionless form by setting $y(x)=\widetilde{\psi_{3}}(S=\ell(1-x))$. We obtain

$$
y^{\prime \prime}(x)+\lambda x^{4} y(x)=0 \quad y^{\prime}(0)=0 \quad y(1)=0
$$

where

$$
\lambda=\frac{\left[\frac{1}{2} \varrho g \ell^{2}\right]^{2} \ell^{2}}{Y I_{2} \cdot \mu J}=\frac{3(1+\nu)}{24}\left(\frac{\varrho g \ell \cdot \ell^{2}}{Y \frac{a t^{3}}{12}}\right)^{2}=\frac{3(1+\nu)}{24} \gamma^{2} .
$$


Here, we have used the expressions of the initial elastic moduli $Y I_{2}=\frac{Y a t^{3}}{12}$ and $\mu J=\frac{Y a t^{3}}{6(1+\nu)}$ from $(3.2)$, the expression of the base stress $M_{1}^{\dagger}(S)=\frac{1}{2} \varrho g(\ell-S)^{2}$, and the definition of the loading parameter $\gamma$ from equation (4.2).

The solution of the dimensionless eigenvalue problem involves Bessel functions of fractional order. A detailed analysis shows that the lowest eigenvalue is $\lambda=\lambda_{\mathrm{c}}:=41.3048$, corresponding to a critical dimensionless load

as announced earlier in equation (4.4).

$$
\gamma_{\mathrm{c}}=\frac{18.178}{(1+\nu)^{1 / 2}}
$$

As noted above, the linearly elastic model uses the same incremental constitutive laws, equation (D.5), and therefore has the same critical load $\gamma_{c}^{\text {lin }}=\gamma_{c}$, and first reported by Mic99.

\section{D.4. Case of Sadowsky model}

We resume from equations (D.2-D.4), this time using the constitutive laws of the Sadowsky model, which write $M_{2}(S)=\frac{Y I_{2}}{\left(1-\nu^{2}\right)} \kappa_{2}(S)\left(1-\left(\frac{\kappa_{3}(S)}{\kappa_{2}(S)}\right)^{4}\right)$ and $M_{3}(S)=\frac{Y I_{2}}{\left(1-\nu^{2}\right)} \kappa_{3}(S) \cdot 2\left(1+\left(\frac{\kappa_{3}(S)}{\kappa_{2}(S)}\right)^{2}\right)$, where $Y I_{2}=Y \frac{a t^{3}}{12}$ as earlier.

In the base solution, both $\kappa_{2}(S)$ and $\kappa_{3}(S)$ are identically zero and $M_{2}(S)$ and $M_{3}(S)$ are not set by the constitutive law, but by the equilibrium, see section D.1.

In the perturbed stated, where both $\kappa_{2}(S)$ and $\kappa_{3}(S)$ are small, the stress is given by the constitutive law as $\widetilde{M_{2}}(S)=M_{2}(S)=\frac{Y I_{2}}{\left(1-\nu^{2}\right)} \tilde{\kappa_{2}}(S)\left(1-\left(\frac{\tilde{\kappa_{3}}(S)}{\tilde{\kappa_{2}}(S)}\right)^{4}\right)$ and $\widetilde{M_{3}}(S)=M_{3}(S)=\frac{Y I_{2}}{\left(1-\nu^{2}\right)} \tilde{\kappa_{3}}(S) \cdot 2\left(1+\left(\frac{\tilde{\kappa_{3}}(S)}{\tilde{\kappa_{2}}(S)}\right)^{2}\right)$. This can be rewritten as

where

$$
\begin{aligned}
\widetilde{M}_{2}(S) & =\frac{Y I_{2}}{\left(1-\nu^{2}\right)} \tilde{\kappa_{2}}(S)\left(1-\eta^{\dagger^{4}}(S)\right) \\
\widetilde{M}_{3}(S) & =\frac{Y I_{2}}{\left(1-\nu^{2}\right)} \tilde{\kappa_{2}}(S) \cdot 2 \eta^{\dagger}(S)\left(1+\eta^{\dagger^{2}}(S)\right)
\end{aligned}
$$

$$
\eta^{\dagger}(S)=\frac{\tilde{\kappa_{3}}(S)}{\tilde{\kappa_{2}}(S)}
$$

is defined as the ratio of the perturbed strains $\tilde{\kappa_{3}}$ and $\tilde{\kappa_{2}}$ : even though it is defined in terms of the perturbations, this quantity $\eta^{\dagger}(S)$ is finite, being the ratio of two perturbed quantities. This points to the fact that the Sadowsky model is non-smooth near planar configurations.

Inserting the constitutive law into equations (D.2) and (D.3), and using the expressions (D.1) of the linearized strain as earlier, we obtain

$$
\begin{gathered}
\frac{\mathrm{d}}{\mathrm{d} S}\left[\frac{\mathrm{d} \widetilde{\psi}_{3}}{\mathrm{~d} S} \cdot 2\left(1+\eta^{\dagger^{2}}(S)\right)\right]-\left(\frac{\left(1-\nu^{2}\right) M_{1}^{\dagger}(S)}{Y I_{2}}\right) \frac{\mathrm{d} \widetilde{\psi}_{2}}{\mathrm{~d} S}=0 \\
\frac{\mathrm{d} \widetilde{\psi}_{2}}{\mathrm{~d} S}\left(1-\eta^{\dagger^{4}}(S)\right)=-\left(\frac{\left(1-\nu^{2}\right) M_{1}^{\dagger}(S)}{Y I_{2}}\right) \widetilde{\psi}_{3}(S)
\end{gathered}
$$

Using (D.7) and (D.1) we replace $\frac{\mathrm{d} \widetilde{\psi}_{2}}{\mathrm{~d} S}$ in (D.8) and (D.9), which both become homogeneous and linear in $\widetilde{\psi_{3}}(S)$ and $\frac{\mathrm{d} \widetilde{\psi}_{3}}{\mathrm{~d} S}$. The determinant condition of this systems reads:

$$
(\ell-S)\left(1+\eta^{\dagger^{2}}\right) \eta^{\dagger \prime}-2 \eta^{\dagger}\left(1-\eta^{\dagger^{2}}\right)-\frac{(\ell-S)^{3}}{4} \frac{\left(1-\nu^{2}\right) \gamma}{\ell^{3}}=0
$$

Boundary condition $\widetilde{\psi}_{3}(0)=0$ with $(\mathrm{D} .2)$ and (D.6) yields either $\tilde{\kappa}_{2}(0)=0$ or $\eta^{\dagger}(0)= \pm 1$. Boundary condition $\widetilde{M}_{3}(\ell)=0$ with (D.6) yields either $\tilde{\kappa_{2}}(\ell)=0$ or $\eta^{\dagger}(\ell)=0$. Among the different possible cases, we find that only $\eta^{\dagger}(0)=-1$ with $\eta^{\dagger}(\ell)=0$ is possible. We then have a boundary value problem which can written in dimensionless form using $y(x)=\eta^{\dagger}(\ell x)$ :

$$
(1-x)\left(1+y^{2}\right) y^{\prime}-2 y\left(1-y^{2}\right)-\left[\left(1-\nu^{2}\right) \gamma\right] \frac{(1-x)^{3}}{4}=0 \quad y(0)=-1 \quad y(1)=0
$$

We solve it numerically and find the lowest $\gamma$ solution to be

This corresponds to

$$
\left[\left(1-\nu^{2}\right) \gamma\right]_{\mathrm{c}}=21.491
$$

as announced earlier in equation (4.4).

$$
\gamma_{\mathrm{c}}^{\mathrm{sdw}}=\frac{21.491}{1-\nu^{2}}
$$

\section{Appendix E. Linear bifurcation analysis of a tWisted ribbon}

This section carries out a linear bifurcation analysis of a twisted ribbon, and proves the results announced in equations (5.1-5.3). We consider a ribbon held by a tension $T$ and subjected to a twisting strain $\tau$. We work in the infinite length limit where the tension and the twist are aligned with the tangent at both extremities. These boundary conditions at infinity for the non-linear problem imply that

$$
\boldsymbol{R}(S)=T \boldsymbol{e}_{z}, \boldsymbol{M}(S)+\boldsymbol{r}(S) \times T \boldsymbol{e}_{z}=\mathbf{0}
$$




\section{E.1. Base solution}

Variables in the base solution are denoted with $(\cdot)^{\dagger}$. This base solution is straight with uniform twist strain $\tau$. We have $\boldsymbol{r}^{\dagger}(S)=S \boldsymbol{e}_{z}, \boldsymbol{d}_{1}^{\dagger}(S)=\boldsymbol{e}_{r}(\tau S), \boldsymbol{d}_{2}^{\dagger}(S)=\boldsymbol{e}_{\theta}(\tau S), \boldsymbol{d}_{3}^{\dagger}(S)=\boldsymbol{e}_{z}$ where $\boldsymbol{e}_{r}(\theta)=\cos \theta \boldsymbol{e}_{x}+\sin \theta \boldsymbol{e}_{y}$ and $\boldsymbol{e}_{\theta}(\theta)=-\sin \theta \boldsymbol{e}_{x}+\cos \theta \boldsymbol{e}_{y}$ is the cylindrical basis in the $\left(\boldsymbol{e}_{x}, \boldsymbol{e}_{y}\right)$ plane. The corresponding strain is

$$
\left(\kappa_{1}^{\dagger}, \kappa_{2}^{\dagger}, \kappa_{3}^{\dagger}\right)=(0,0, \tau)
$$

The longitudinal traction force is applied at the remote endpoints, $\boldsymbol{R}^{\dagger}(S)=T \boldsymbol{e}_{z}$. The rotation gradient is constant, $\boldsymbol{\kappa}^{\dagger}(S)=\tau \boldsymbol{e}_{z}$. Using the constitutive law, the pre-stress is a twisting moment $\boldsymbol{M}^{\dagger}(S)=M_{3}^{\dagger} \boldsymbol{e}_{z}$ where the twisting moment $M_{3}^{\dagger}$ is constant,

$$
M_{3}^{\dagger}=\frac{\partial W}{\partial \kappa_{3}}(0, \tau) \text {. }
$$

\section{E.2. Linearized equations}

We reuse the general linearization from appendix C. The perturbed solution is denoted with $\widetilde{(\cdot)}$. The perturbed displacement is decomposed in the unperturbed basis as $\tilde{\boldsymbol{r}}(S)=\tilde{r}_{i}(S) \boldsymbol{d}_{i}^{\dagger}(S)$. We project equation (C.3) onto $\boldsymbol{d}_{1}^{\dagger}$ and $\boldsymbol{d}_{2}^{\dagger}$

$$
\begin{aligned}
& \tilde{r}_{1}^{\prime}(S)-\tau \tilde{r_{2}}(S)=\widetilde{\psi_{2}}(S) \\
& \tilde{r}_{2}^{\prime}(S)+\tau \tilde{r_{1}}(S)=-\widetilde{\psi_{1}}(S)
\end{aligned}
$$

The expressions of the perturbed strain in equation (C.1) yields

$$
\begin{aligned}
& \tilde{\kappa_{1}}(S)=\tilde{\psi}_{1}^{\prime}(S)-\tau \widetilde{\psi_{2}}(S) \\
& \tilde{\kappa_{2}}(S)=\tilde{\psi}_{2}^{\prime}(S)+\tau \widetilde{\psi_{1}}(S)
\end{aligned}
$$

Equation (C.5) connects the perturbations to the bending and twisting moments $\widetilde{M}_{i}$ and the perturbation to the internal moment $\tilde{M}$; for the problem at hand, it reads

$$
\tilde{\boldsymbol{M}}(S)=\left(\widetilde{M}_{1}(S)+M_{3}^{\dagger} \widetilde{\psi}_{2}(S)\right) \boldsymbol{d}_{1}^{\dagger}(S)+\left(\widetilde{M}_{2}(S)-M_{3}^{\dagger} \widetilde{\psi}_{1}(S)\right) \boldsymbol{d}_{2}^{\dagger}(S)+\widetilde{M}_{3}(S) \boldsymbol{d}_{3}^{\dagger}(S)
$$

As we have $\boldsymbol{R}^{\dagger}(S)=\boldsymbol{R}(S)=T \boldsymbol{e}_{z}$, there is no perturbation to the internal force $\tilde{\boldsymbol{R}}(S)=\mathbf{0}$. With $\tilde{\boldsymbol{R}}(S)=\mathbf{0}$ and $\boldsymbol{R}^{\dagger}(S)=T \boldsymbol{e}_{z}$, the balance of moments in equation (C.4) reads $\tilde{\boldsymbol{M}}^{\prime}(S)+\tilde{\boldsymbol{r}}^{\prime}(S) \times T \boldsymbol{e}_{z}=0$, which shows that $\tilde{\boldsymbol{M}}(S)+\tilde{\boldsymbol{r}}(S) \times T \boldsymbol{e}_{z}$ is constant. Using (E.1) we find this constant to be zero, $\tilde{\boldsymbol{M}}(S)+\tilde{\boldsymbol{r}}(S) \times T \boldsymbol{e}_{z}=\mathbf{0}$. When this equation is projected along $\boldsymbol{d}_{2}^{\dagger}$ we obtain

$$
\widetilde{M_{2}}(S)=M_{3}^{\dagger} \widetilde{\psi_{1}}(S)+T \tilde{r_{1}}(S) .
$$

Linearizing the constitutive law $M_{2}=\frac{\partial W}{\partial \kappa_{2}}\left(\kappa_{2}, \kappa_{3}\right)$ about $\left(\kappa_{2}^{\dagger}, \kappa_{3}^{\dagger}\right)=(0, \tau)$, we find $\widetilde{M_{2}}=\frac{\partial^{2} W}{\partial \kappa_{2}^{2}}(0, \tau) \tilde{\kappa_{2}}+\frac{\partial^{2} W}{\partial \kappa_{2} \partial \kappa_{3}}(0$, $\tau) \tilde{\kappa_{3}}$. Since $W\left(\kappa_{2}, \kappa_{3}\right)$ is an even function of $\kappa_{2}$, we have $\frac{\partial^{2} W}{\partial \kappa_{2} \partial \kappa_{3}}(0, \tau)=0$. Denoting as $K_{2}^{\dagger}$ the incremental bending
modulus,

$$
K_{2}^{\dagger}=\frac{\partial^{2} W}{\partial \kappa_{2}^{2}}(0, \tau),
$$

we write the constitutive constraint and the incremental constitutive law in bending together as

$$
\begin{aligned}
0 & =\tilde{\kappa_{1}} \\
\widetilde{M_{2}} & =K_{2}^{\dagger} \tilde{\kappa_{2}} .
\end{aligned}
$$

Inserting equations (E.5) and (E.6) into the constitutive law (E.8), we find

$$
\begin{aligned}
\tilde{\psi}_{1}^{\prime}(S)-\tau \widetilde{\psi_{2}}(S) & =0 \\
\tilde{\psi}_{2}^{\prime}(S)+\tau \widetilde{\psi_{1}}(S) & =\frac{1}{K_{2}^{\dagger}}\left(M_{3}^{\dagger} \widetilde{\psi}_{1}(S)+T \tilde{r_{1}}(S)\right)
\end{aligned}
$$

In view of the invariance of the base solution in the axial direction, we seek the buckling mode as a pure Fourier mode: denoting as $\hat{r}_{1}, \hat{r}_{2}, \hat{\psi}_{1}$ and $\hat{\psi}_{2}$ the unknown complex amplitudes, we write $\tilde{r}_{j}(S)=\mathrm{e}^{\mathrm{i} q S} \hat{r}_{j}$ and $\tilde{\psi}_{j}(S)=\mathrm{e}^{\mathrm{i} q S} \hat{\psi}_{j}$. Equations (E.4) and (E.9) then yields four homogeneous linear equations for the complex amplitudes, which can be rewritten in matrix form as

$$
\left(\begin{array}{cccc}
0 & 0 & i q & -\tau \\
-\frac{T}{K_{2}^{\dagger}} & 0 & \tau-\frac{M_{3}^{\dagger}}{K_{2}^{\dagger}} & i q \\
i q & -\tau & 0 & -1 \\
\tau & i q & +1 & 0
\end{array}\right) \cdot\left(\begin{array}{c}
\hat{r}_{1} \\
\hat{r}_{2} \\
\hat{\psi}_{1} \\
\hat{\psi}_{2}
\end{array}\right)=\mathbf{0}
$$

Given the applied tension $T$, the problem is to find critical values of the twisting strain $\tau$ and a wavenumber $q$ such that the above problem has non-trivial solutions. In the matrix, recall that $M_{3}^{\dagger}$ and $K_{2}^{\dagger}$ are functions of $\tau$, see equations (E.3) and (E.7). 
The existence of a non-trivial solution to the above linear problem requires that the determinant of the matrix is zero. After some algebra, this condition can be rewritten as

$$
\left(q^{2}-\left(\tau^{2}-\frac{T+M_{3}^{\dagger} \tau}{2 K_{2}^{\dagger}}\right)\right)^{2}+2 \tau^{2} \frac{T}{K_{2}^{\dagger}}-\left(\frac{T+M_{3}^{\dagger} \tau}{2 K_{2}^{\dagger}}\right)^{2}=0 .
$$

For given values of the loading $T$ and the twisting strain $\tau$, either this condition is satisfied for some $q$ and we have a marginally stable configuration (incipient buckling), or it is not satisfied for any $q$ and the configuration is not marginally stable (no buckling).

To analyze the dispersion equation above, we introduce the dimensionless quantities,

This yields

$$
k=\frac{q}{\sqrt{T / K_{2}^{\dagger}}} \quad t^{\dagger}=\frac{\tau^{2}}{T / K_{2}^{\dagger}} \quad m^{\dagger}=\frac{M_{3}^{\dagger} \tau}{T}
$$

$$
\left(k^{2}-\left(t^{\dagger}-\frac{1+m^{\dagger}}{2}\right)\right)^{2}+2 t^{\dagger}-\left(\frac{1+m^{\dagger}}{2}\right)^{2}=0 .
$$

The left-hand side goes to $+\infty$ for $k \rightarrow \pm \infty$. Therefore, the condition for the existence of at least a value of the wavenumber $k$ such that the equation above is verified is

$$
\min _{k \in \mathbb{R}}\left[\left(k^{2}-\left(t^{\dagger}-\frac{1+m^{\dagger}}{2}\right)\right)^{2}\right]+2 t^{\dagger}-\left(\frac{1+m^{\dagger}}{2}\right)^{2} \leqslant 0
$$

The minimum can be calculated explicitly,

$$
\min _{k \in \mathbb{R}}\left[\left(k^{2}-a\right)^{2}\right]=\left\{\begin{array}{lll}
0 & \text { if } a \geqslant 0 & \text { (optimum: } k= \pm \sqrt{a}) \\
a^{2} & \text { if } a<0 & \text { (optimum: } k=0)
\end{array}\right.
$$

This yields the bifurcation criterion as

$$
0 \geqslant \begin{cases}2 t^{\dagger}-\left(\frac{1+m^{\dagger}}{2}\right)^{2} & \text { if } t^{\dagger} \geqslant \frac{1+m^{\dagger}}{2} \\ \left(t^{\dagger}-\frac{1+m^{\dagger}}{2}\right)^{2}+2 t^{\dagger}-\left(\frac{1+m^{\dagger}}{2}\right)^{2} & \text { if } t^{\dagger}<\frac{1+m^{\dagger}}{2}\end{cases}
$$

The first critical load is when the quantity in the large parenthesis is equal to zero. Simplifying the second alternative by elementary algebra, we arrive at the bifurcation condition

$$
0= \begin{cases}2 t^{\dagger}-\left(\frac{1+m^{\dagger}}{2}\right)^{2} & \text { if } 2 t^{\dagger}-m^{\dagger} \geqslant 1 \\ t^{\dagger}\left(1-\left(m^{\dagger}-t^{\dagger}\right)\right) & \text { if } 2 t^{\dagger}-m^{\dagger}<1\end{cases}
$$

Clearly, no instability is possible in the absence of twisting $\left(\tau=0\right.$, implying $\left.t^{\dagger}=0\right)$, which suggests that the root $t^{\dagger}=0$ in the second alternative corresponds to a ghost buckling mode; it can indeed be verified that the corresponding mode is a rigid-body translation. We remove this ghost mode by dividing the function in the parenthesis by $t^{\dagger}$ :

$$
0= \begin{cases}2-\frac{1}{t^{\dagger}}\left(\frac{1+m^{\dagger}}{2}\right)^{2} & \text { if } 2 t^{\dagger}-m^{\dagger} \geqslant 1 \\ 1-\left(m^{\dagger}-t^{\dagger^{2}}\right) & \text { if } 2 t^{\dagger}-m^{\dagger}<1\end{cases}
$$

As noted in equation (E.11), the first alternative, for $t^{\dagger} \geqslant \frac{1+m^{\dagger}}{2}$, corresponds to a finite-wavelength instability, $k= \pm \sqrt{t^{\dagger}-\frac{1+m^{\dagger}}{2}}$ in the helical frame $\boldsymbol{d}_{i}^{\dagger}(S)$; the second alternative, for $t^{\dagger}<\frac{1+m^{\dagger}}{2}$ corresponds to an infinitewavelength instability, $k=0$. Equation (E.12) proves the result announced earlier in equations (5.1-5.2). In view of equations (E.3), (E.7) and (E.10), the quantities appearing in equation (E.12) are

$$
t^{\dagger}=\frac{\tau^{2}}{T} \frac{\partial^{2} W}{\partial \kappa_{2}{ }^{2}}(0, \tau) \quad m^{\dagger}=\frac{\tau \frac{\partial W}{\partial \kappa_{3}}(0, \tau)}{T}
$$

